\title{
Well-Construction, Water-Level, and Ground- Water-Quality Data for Prince William County, Virginia, 1992
}

By DAVID L. NELMS and ALLEN R. BROCKMAN

U.S. GEOLOGICAL SURVEY

Open-File Report 93-443

Prepared in cooperation with the

PRINCE WILLIAM HEALTH DISTRICT

Richmond, Virginia

1993 


\title{
U.S. DEPARTMENT OF THE INTERIOR BRUCE BABBITT, Secretary
}

\author{
U.S. GEOLOGICAL SURVEY \\ ROBERT M. HIRSCH, Acting Director
}

For additional information write to:

Copies of this report may be purchased from:

District Chief

U.S. Geological Survey

3600 West Broad Street, Room 606

Richmond, VA 23230
U.S. Geological Survey

Earth Science Information Center

Open-File Reports Section

Box 25286, MS 517

Denver Federal Center

Denver, CO 80225 


\section{CONTENTS}

Introduction $\ldots \ldots \ldots \ldots \ldots \ldots \ldots \ldots \ldots \ldots \ldots \ldots \ldots \ldots \ldots \ldots \ldots \ldots$

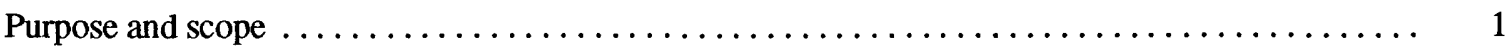

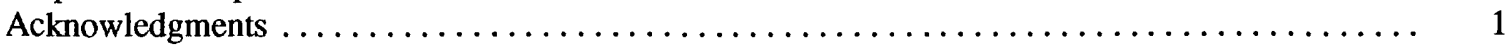

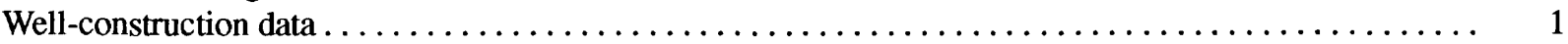

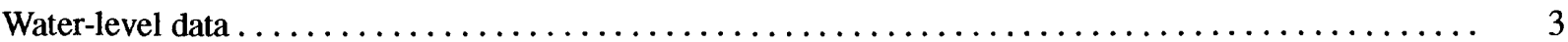

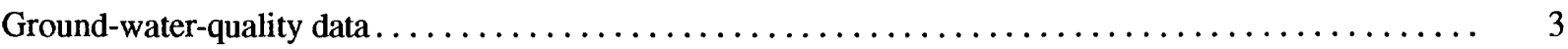

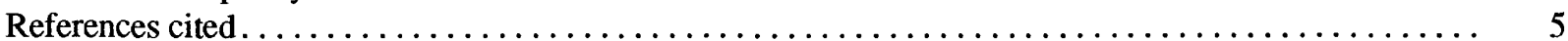

\section{PLATE}

1. Map showing location of wells used for water-level measurements and ground-waterquality samples In pocket

\section{FIGURES}

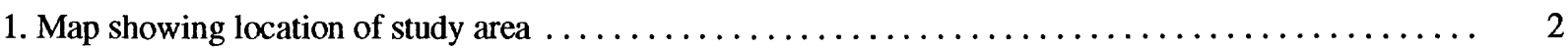

2. Hydrographs for observation wells 49U1, 49V1, 51S7, 52S4, 52S5, and 53T2 SOW $029 \ldots \ldots$

\section{TABLES}

1. Well-construction data for selected wells in Prince William County . . . . . . . . . . . . . . 9

2. Synoptic water-level measurements for selected wells in Prince William County . . . . . . . . . . 44

3. Major dissolved constituent and nutrient analyses of ground water in Prince William County . . . . . 52

4. Trace-metal analyses of ground water in Prince William County $\ldots \ldots \ldots \ldots \ldots$

5. Environmental isotope analyses of ground water in Prince William County $\ldots \ldots \ldots \ldots$

6. Dissolved gas analyses of ground water in Prince William County $\ldots \ldots \ldots \ldots$ 


\title{
Well-Construction, Water-Level, and Ground- Water-Quality Data for Prince William County, Virginia, 1992
}

\author{
By David L. Nelms and Allen R. Brockman
}

\section{INTRODUCTION}

The U.S. Geological Survey (USGS), in cooperation with the Prince William Health District, conducted an investigation between 1989 and 1992 to characterize the quality of ground water in Prince William County, Va. Encompassing approximately $347 \mathrm{mi}^{2}$, Prince William County is located in northern Virginia, about $25 \mathrm{mi}$ southwest of Washington, D.C. (fig. 1).

\section{Purpose and Scope}

This report presents well-construction, waterlevel, and ground-water-quality data collected by the USGS between 1989 and 1992 in Prince William County. Hydrogeologic data were collected as part of an investigation to assess the quality of ground water in the county. Well-construction data for 182 sites were entered into the Ground-Water Site Inventory (GWSI) data base maintained by the USGS. Water levels were measured in 159 wells in the spring and fall of 1991 and the data also were entered into the GWSI. Hydrographs from six of these wells also are presented in this report. Analyses are included for 88 ground-water-quality samples and are stored in the Water-Quality System data base maintained by the USGS. In addition, analyses for environmental or stable isotopes from 57 wells and dissolved gas analyses from 18 wells are presented.

\section{Acknowledgments}

The authors wish to express their appreciation to James Shifflett and Frank McDonough of the city of Manassas Park, Donald Echols and Darrell Grady of the city of Manassas, and Earl Smith and Jim Camper of the Upper Occoquan Sewage Authority for providing access to wells. The assistance of Ralph Eckley, Jim Green, David Miller, and Lee Hefner of the Prince William County Service Authority during the different phases of this investigation was extremely beneficial. Dean W. Chartrand of the International Business Machines Corporation provided valuable water-level data for this investigation. The authors would like to thank Marcus Haynes of the Prince William Health District for his input throughout this investigation. Special thanks also are expressed to the citizens of Prince William County who allowed access to their wells.

\section{WELL-CONSTRUCTION DATA}

Well-construction data for 182 sites used in this investigation for water-level measurements and ground-water-quality samples are presented in table 1 . The well-construction data were compiled from Cady (1938), Brown (1981), Betz-ConverseMurdoch (1982), Posner and Zenone (1983), Nelms and Richardson (1990), and files maintained by the Virginia Department of Environmental Quality, Water Division (DEQ, WD), the Prince William Health District, and the USGS. For location of these wells, the reader can refer to plate 1. 


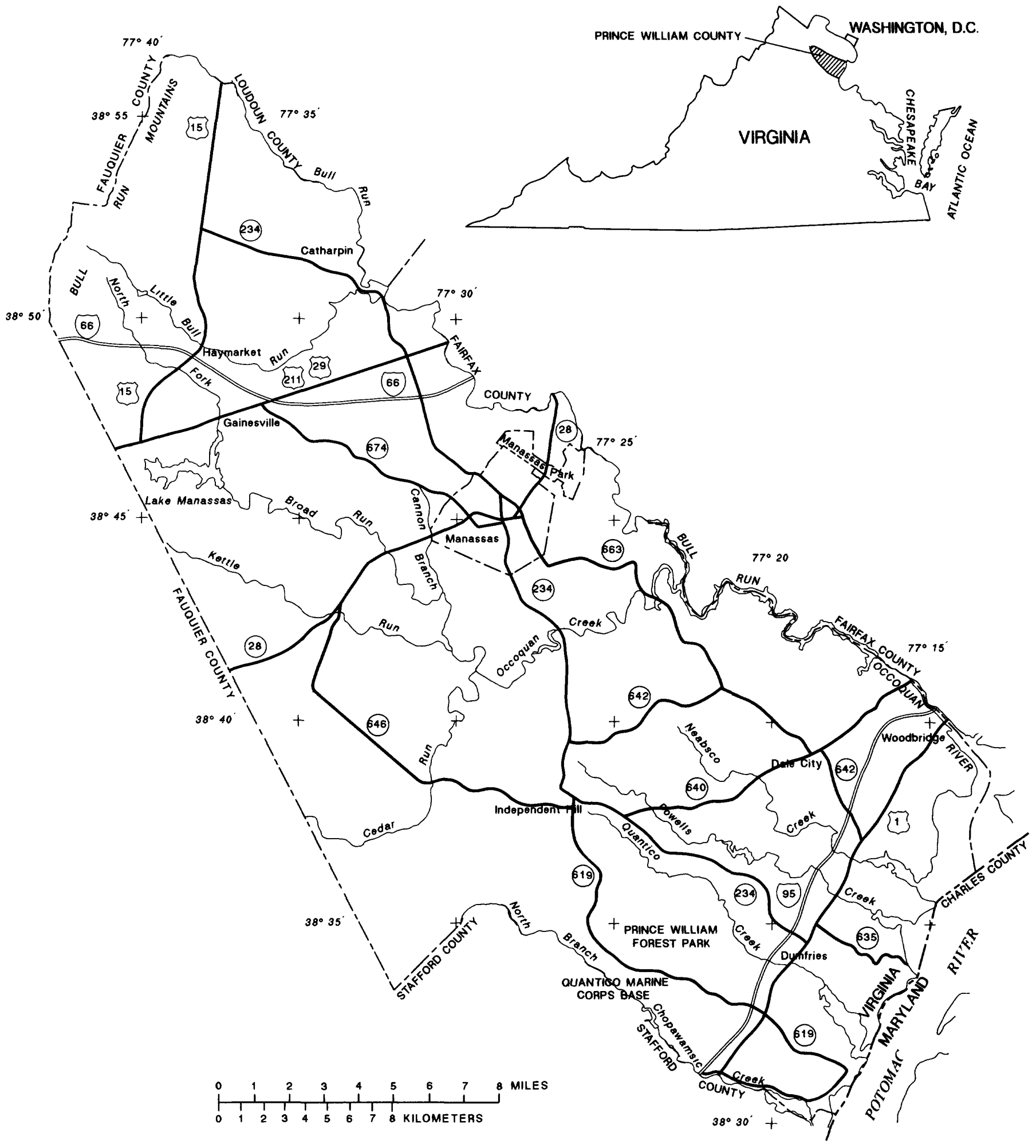

Figure 1. Location of study area. 


\section{WATER-LEVEL DATA}

Water levels were measured in 159 wells during two synoptic runs in the spring and fall of 1991 to approximate the seasonal water-level fluctuations (table 2). The International Business Machines Corporation provided water-level data for 41 of these wells. Pumps were shut down for as long as feasible and the recovery period was recorded. During the fall synoptic run, the recovery duration of the spring run was duplicated whenever possible. At least three water-level measurements were collected at a site during each synoptic run. At least two measurements had to be identical for the water level in table 2 to be reported as a static level. For wells that had not completely recovered, a series of measurements were collected until the difference between measurements was less than $0.5 \mathrm{ft}$; therefore, the water level reported in table 2 is an approximate static level. The site status heading in table 2 indicates the type of static level reported.

Six observation wells located in Prince William County are part of the observation-well network for the State of Virginia maintained by the USGS and DEQ, WD. Of these six wells, the USGS maintains observation wells 49U1, 49V1, 51S7, 52S4, and 52S5. Observation well 53T2 SOW 029 is maintained by the DEQ, WD. Hydrographs for each well showing the entire period of record are presented in figure 2 .

\section{GROUND-WATER-QUALITY DATA}

Ground-water-quality samples were collected at 87 wells in the county during the summer months of 1990 and 1991 to characterize the quality of ground water. Procedures outlined in Wood (1976), Scalf and others (1981), and Claassen (1982) were followed during sample collection. Field properties ( $\mathrm{pH}$, specific conductance, dissolved oxygen, and temperature) were monitored in a flow chamber during the purging of each well. Domestic wells were purged either for at least 1 hour or until the field properties had stabilized after the first hour. Public-supply wells generally had been pumped for several hours and were effec- tively purged prior to the arrival of project personnel; therefore, field properties at these wells were monitored for at least an additional 30 minutes or until stabilized. Filtration or treatment devices were bypassed, so that a representative water sample from the aquifer could be collected.

Samples collected for major cation, trace metal, and nutrient analyses were filtered through a $0.45 \mu$ (micron) -membrane filter. Dissolved organic carbon samples were filtered through a $0.45 \mu$-silver filter. Alkalinity was measured in the field by use of the fixed endpoint ( $\mathrm{pH}=4.5$ ), electrometric titration method. Radon-222 samples were collected from the flow chamber by use of the syringe method described in Cecil and Yang (1987). The dissolved gas samples were collected in glass flasks following the method described in Pearson and others (1978).

Major dissolved constituent and nutrient analyses of ground water, including radon-222 and dissolved organic carbon, are presented in table 3. Analyses of trace metals in ground water are presented in table 4 . Well 49V53 has two entries in tables 3,4 , and 5 . The first entry is for the sample collected when the well was flowing. The second entry is for the sample collected when the well was pumped. Environmental or stable isotope analyses for 57 wells are presented in table 5. Dissolved gas analyses of ground water from 18 wells are presented in table 6.

Ground-water-quality samples were analyzed at the USGS National Water Quality Laboratory in Denver, Colo. The environmental isotope and dissolved gas samples were analyzed in the laboratories maintained by the USGS National Research Program in Reston, Va. Methods for determination of inorganic and organic constituents in water used by the laboratory are documented in Fishman and Friedman (1989) and Wershaw and others (1987). Laboratory quality-assurance procedures are outlined in Friedman and Erdmann (1982) and Jones (1987). 

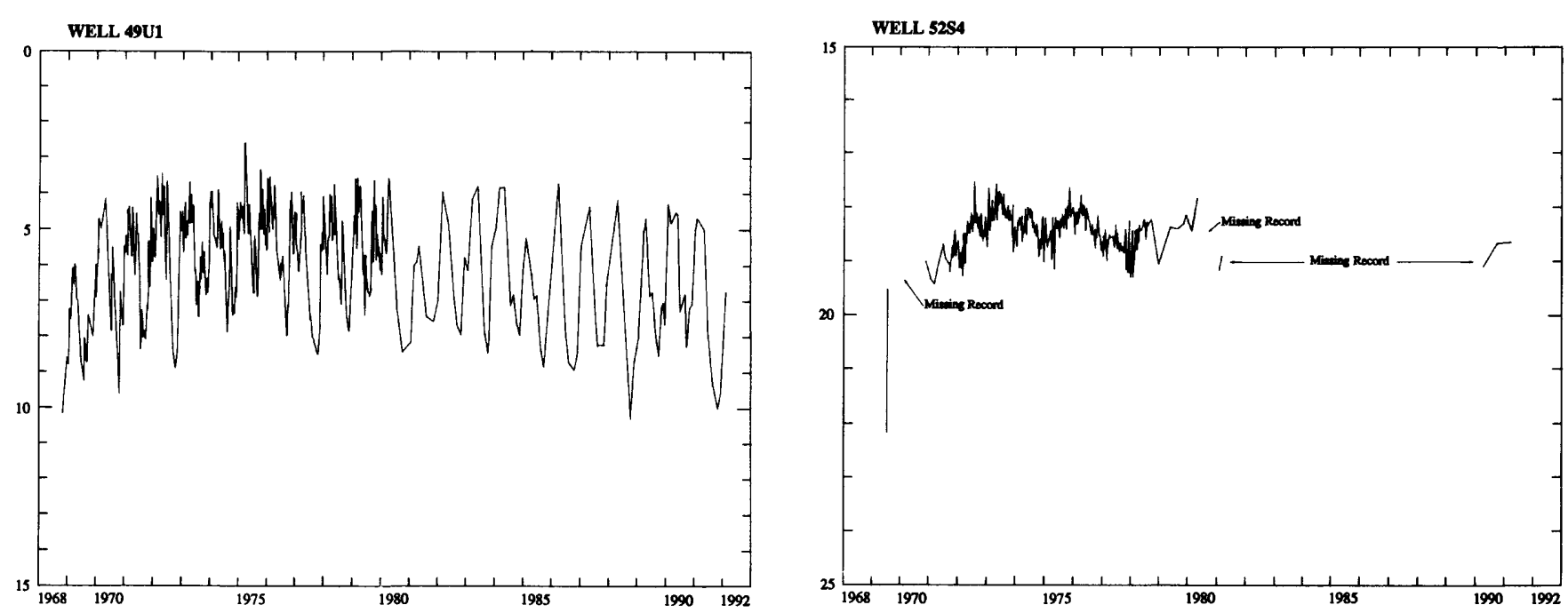

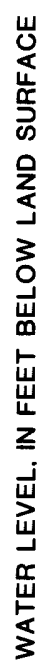
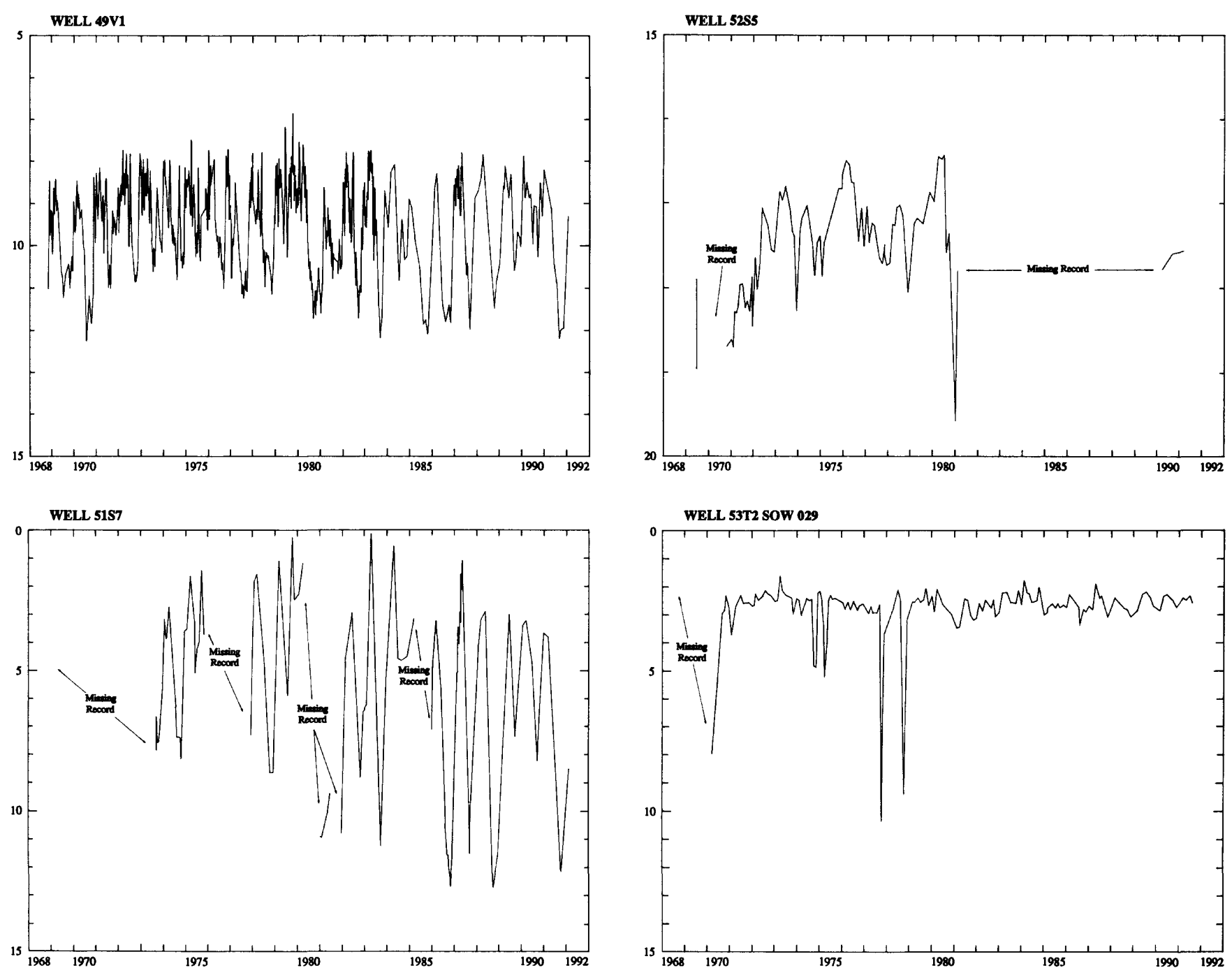

Figure 2. Hydrographs for observation wells 49U1, 49V1, 51S7, 52S4, 52S5, and 53T2 SOW 029. 


\section{REFERENCES CITED}

Betz-Converse-Murdoch, Inc., 1982, Groundwater supply study for Prince William County, Virginia: $65 \mathrm{p}$.

Brown, G.A., 1981, Water resources of Prince William Forest Park, Virginia: U.S. Geological Survey OpenFile Report 80-964, 2 sheets, scale 1:24,000.

Cady, R.C., 1938, Ground-water resources of northern Virginia: Virginia Geological Survey Bulletin 50, $200 \mathrm{p}$.

Cecil, L.D., and Yang, Al, 1987, Guidelines for sampling and analysis for dissolved radon-222 in ground water and surface water: U.S. Geological Survey Office of Water Quality Technical Memorandum no. 88.02 .

Claassen, H.C., 1982, Guidelines and techniques for obtaining water samples that accurately represent the water chemistry of an aquifer: U.S. Geological Survey Open-File Report 82- 1024, 49 p.

Comer, C.D., 1976, Prince William County groundwater-Present conditions and prospects: Virginia State Water Control Board, Planning Bulletin 303, 74 p.

Fishman, M.J., and Friedman, L.C., 1989, Methods for determination of inorganic substances in water and fluvial sediments (3d ed.): U.S. Geological Survey Techniques of Water-Resources Investigations, book 5, chap. A1, 545 p.

Friedman, L.C., and Erdmann, D.E., 1982, Quality assurance practices for the chemical and biological analyses of water and fluvial sediments: U.S. Geological Survey Techniques of Water-Resources Investigations, book 5, chap. A6, 181 p.

Jones, B.E., 1987, Quality control manual of the U.S. Geological Survey's National Water Quality Laboratory: U.S. Geological Survey Open-File Report 87-451, $17 \mathrm{p}$.

Nelms, D.L., and Richardson, D.L., 1990, Geohydrology and the occurrence of volatile organic compounds in ground water, Culpeper basin of Prince
William County, Virginia: U.S. Geological Survey Water-Resources Investigations Report 90-4032, $94 \mathrm{p}$.

Pavlides, Louis, 1990, Geology of part of the northern Virginia Piedmont: U.S. Geological Survey OpenFile Report 90-548, 1 sheet, scale 1:100,000.

Pearson, F.J., Jr., Fisher, D.W., and Plummer, L.N., 1978 , Correction of ground-water chemistry and carbon isotopic composition for effects of $\mathrm{CO}_{2}$ outgassing: Geochimica et Cosmochimica Acta, v. 42, p. 1799-1807.

Posner, Alex, and Zenone, Chester, 1983, Chemical quality of ground water in the Culpeper basin, Virginia and Maryland: U.S. Geological Survey Miscellaneous Investigations Series Map I- 1313-D, 1 sheet, scale 1:125,000.

Scalf, M.R., McNabb, J.F., Dunlap, W.J., Cosby, R.L., and Fryberger, J.S., 1981, Manual of ground-water quality sampling procedures: U.S. Environmental Protection Agency, EPA-600/2- 81-160, 93 p.

Trainer, F.W., and Watkins, F.A., Jr., 1975, Geohydrologic reconnaissance of the upper Potomac River basin: U.S. Geological Survey Water-Supply Paper 2035, 68 p.

Wershaw, R.L., Fishman, M.J., Grabbe, R.R., and Lowe, L.E., eds., 1987, Methods for the determination of organic substances in water and fluvial sediments: U.S. Geological Survey Techniques of WaterResources Investigations, book 5, chap. A3, 80 p.

Wood, W.W., 1976, Guidelines for collection and field analysis of ground-water samples for selected unstable constituents: U.S. Geological Survey Techniques of Water-Resources Investigations, book 1 , chap. D2, 24 p. 
TABLES $1-6$

PAGE 9 FOLLOWS 
Table 1. Well-construction data for selected wells in Prince William County

\section{EXPLANATION OF CODES}

Assignor

\begin{tabular}{|c|c|}
\hline BRME & Bull Run Mountain Estates \\
\hline GMSD & Greater Manassas Sanitary District \\
\hline NPS & National Park Service \\
\hline Potomac R Proj & Potomac River Project \\
\hline PWC TAX & $\begin{array}{l}\text { Prince William County } \\
\text { Tax Map number }\end{array}$ \\
\hline PWCSA & $\begin{array}{l}\text { Prince William County } \\
\text { Service Authority }\end{array}$ \\
\hline SWCB & $\begin{array}{l}\text { Virginia Water Control Board } \\
\text { observation-well network }\end{array}$ \\
\hline UOSA & Upper Occoquan Sewage Authority \\
\hline USGS & U.S. Geological Survey \\
\hline VASWCB & Virginia Water Control Board \\
\hline VDMR & $\begin{array}{l}\text { Virginia Division of } \\
\text { Mineral Resources }\end{array}$ \\
\hline VWCB Bull 303 & $\begin{array}{l}\text { Virginia Water Control Board } \\
\text { Planning Bulletin } 303\end{array}$ \\
\hline WSP 2035 & $\begin{array}{l}\text { U.S. Geological Survey } \\
\text { Water-Supply Paper } 2035\end{array}$ \\
\hline $\mathrm{S}$ & Yorkshire Sanitary District \\
\hline
\end{tabular}

Topographic setting

C Stream channel

F Flat

G Flood plain

$\mathrm{H} \quad$ Hilltop

S Hillside

$T$ Terrace

V Valley flat

W Upland draw

Other data available

QW Water quality

WL Water level
Use of water

C Commercial

F Fire

H Domestic

I Irrigation

$\mathrm{N}$ Industrial

P Public supply

$T$ Institutional

$\mathrm{U}$ Unused
Type of finish

G Gravel screen

S Screen

W Walled

X Open hole

$\mathrm{Z}$ Other

\section{Geologic unit}

$\begin{array}{ll}\text { 112CLMB } & \text { Columbia Group } \\ \text { 217PTMC } & \text { Potomac Group } \\ \text { 227CPCK } & \text { Catharpin Creek Formation of the Culpeper Group } \\ \text { 227DIBS } & \text { Diabase } \\ \text { 227GCRK } & \text { Goose Creek Member of the Catharpin Creek Formation } \\ \text { 227HKGV } & \text { Hickory Grove Basalt of the Culpeper Group } \\ \text { 227MBKQ } & \text { Millbrook Quarry Member of the Waterfall Formation } \\ \text { 227MDLD } & \text { Midland Formation of the Culpeper Group } \\ \text { 227MZCC } & \text { Mount Zion Church Basalt of the Culpeper Group } \\ \text { 227SNDR } & \text { Sander Basalt of the Culpeper Group } \\ \text { 227TMMP } & \text { Thermally metamorphosed rocks } \\ \text { 227TRKR } & \text { Turkey Run Formation of the Culpeper Group } \\ \text { 227WRFL } & \text { Waterfall Formation of the Culpeper Group } \\ \text { 231BLBF } & \text { Balls Bluff Siltstone of the Culpeper Group } \\ \text { 231PLVL } & \text { Poolesville Member of the Manassas Sandstone } \\ \text { 377CPMC } & \text { Chopawamsic Formation } \\ \text { 350MTTL } & \text { Metatonalite } \\ \text { 360LGRV } & \text { Lunga Reservoir Formation } \\ \text { 360LKJK } & \text { Lake Jackson Pluton } \\ \text { 360OCCQ } & \text { Occoquan Pluton } 1 \\ \text { 360PCBC } & \text { Purcell Branch Formation } \\ \text { 360PLLT } & \text { Phyllite } \\ \text { 361QNTC } & \text { Quantico Formation } \\ \text { 377CHLH } & \text { Chilhowee Group }\end{array}$

${ }^{1}$ Unit follows usage of U.S. Geological Survey Open-File Report 90-548 (Pavlides, 1990). 


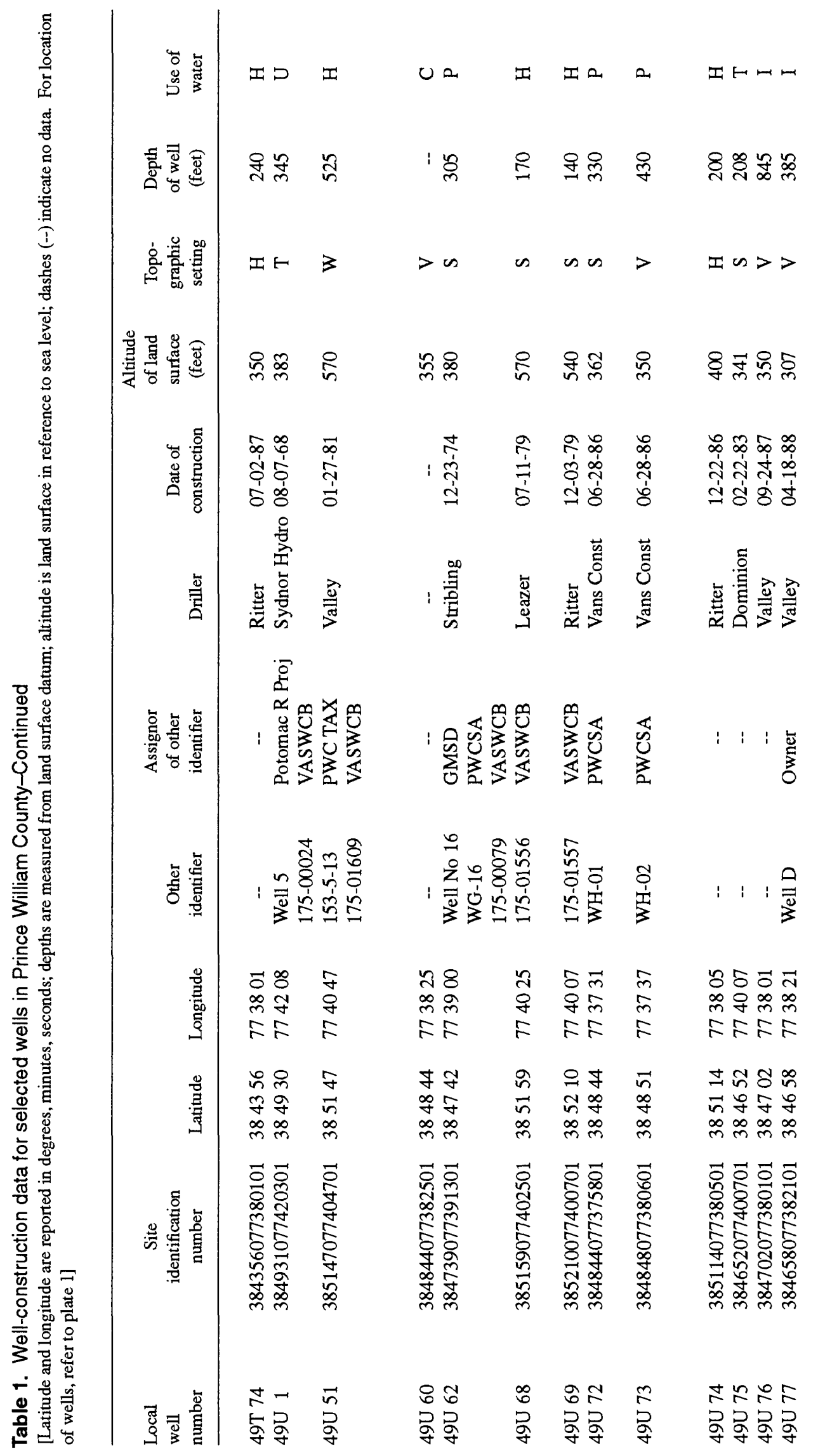




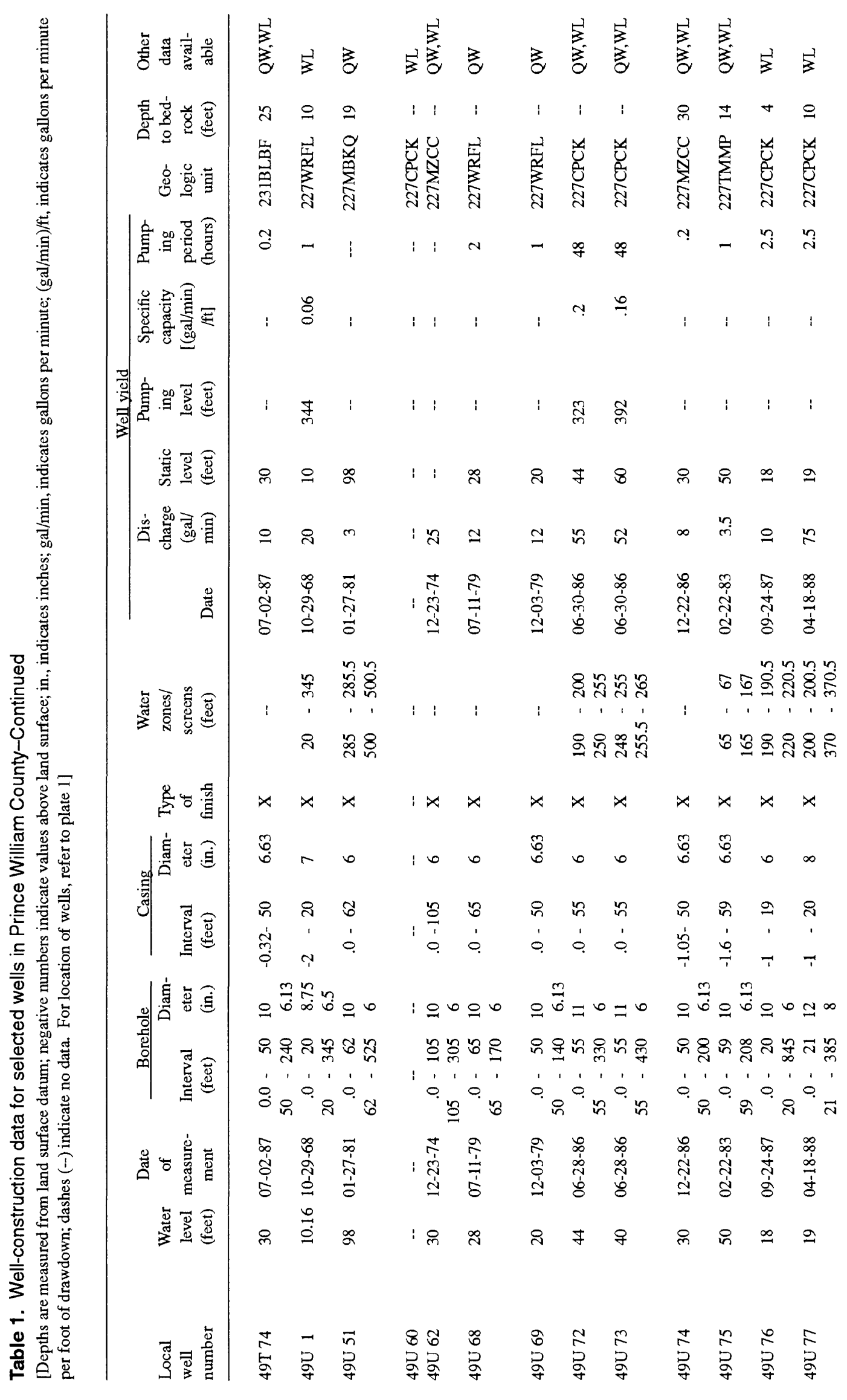




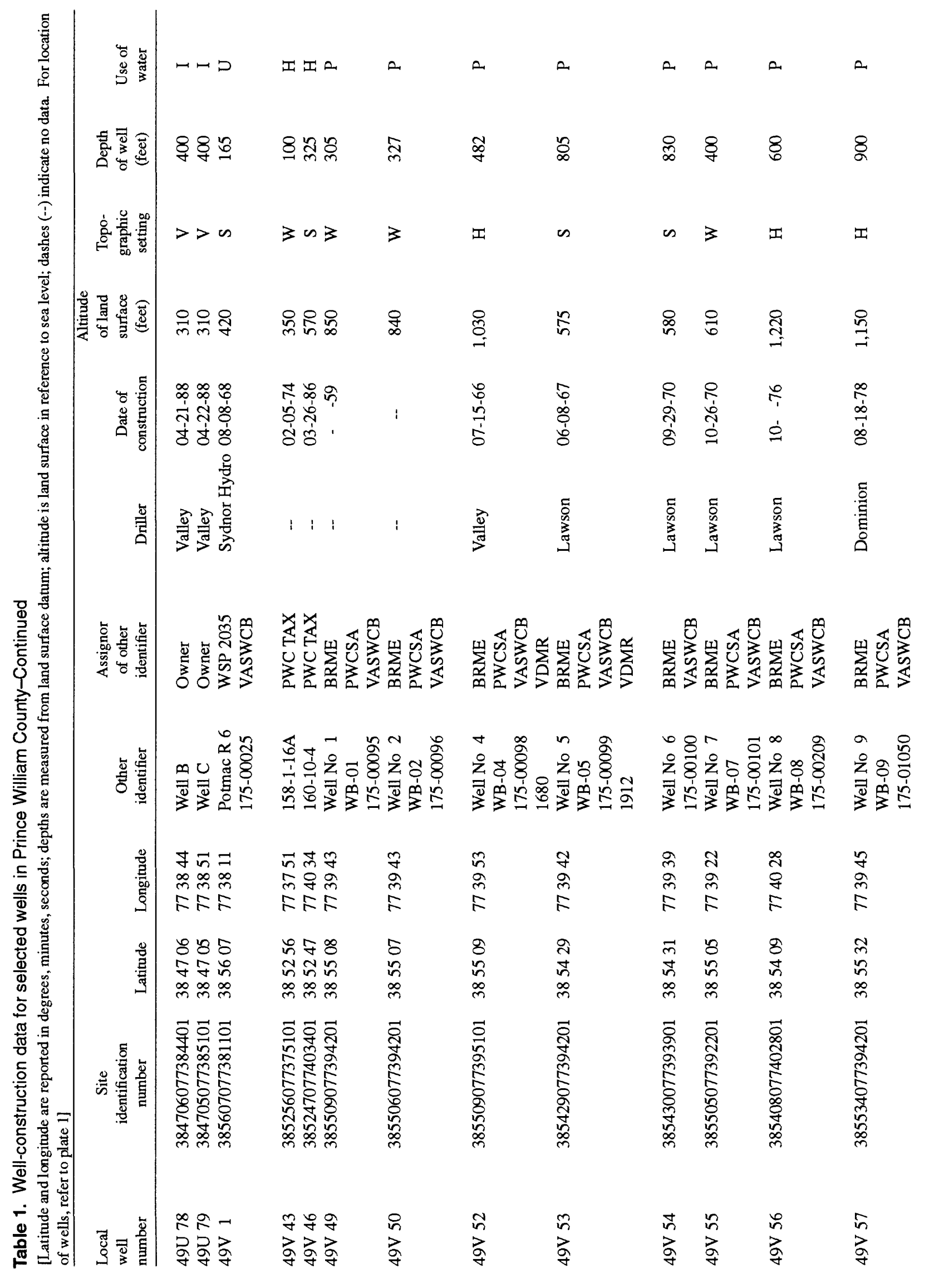




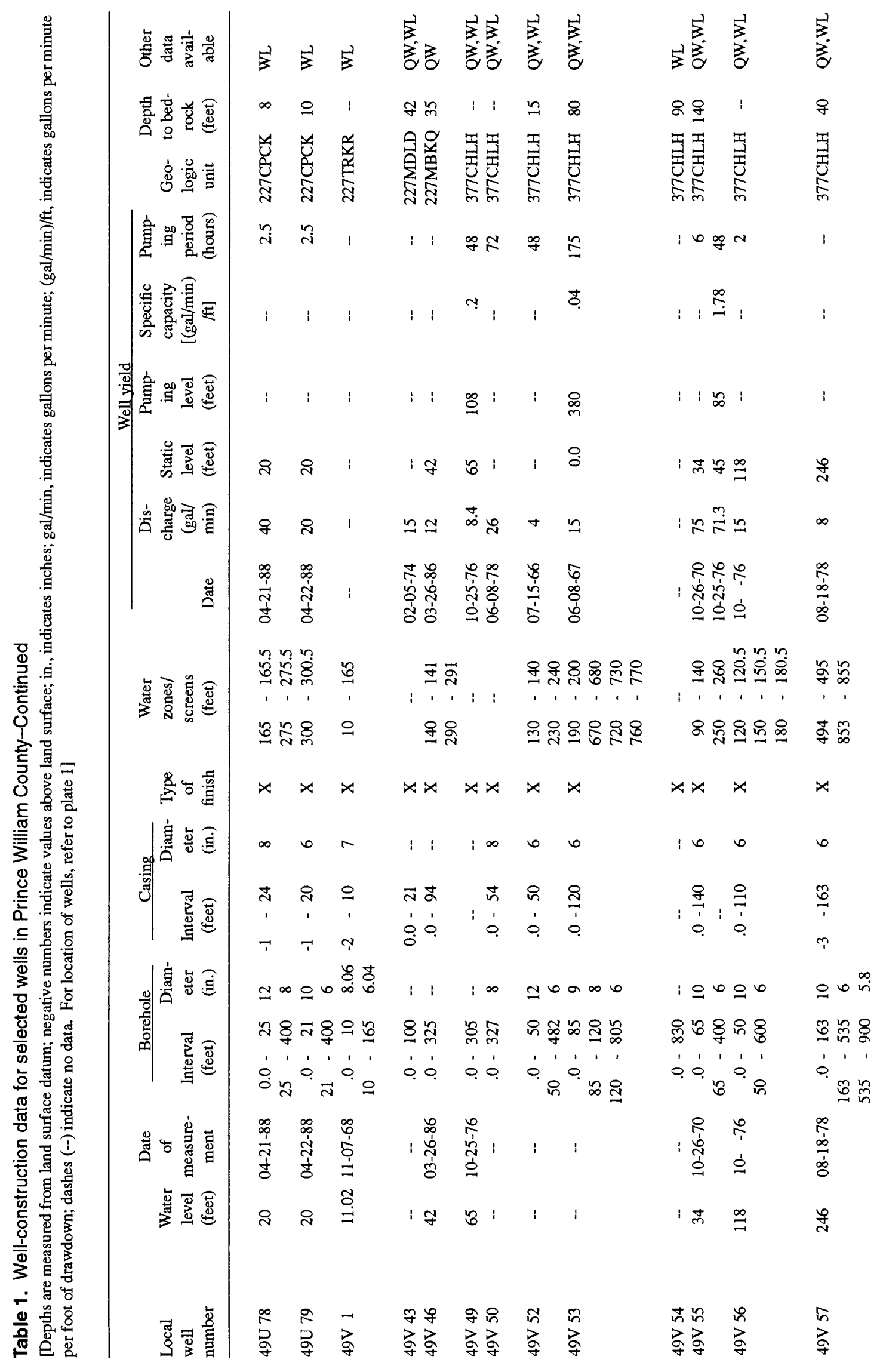




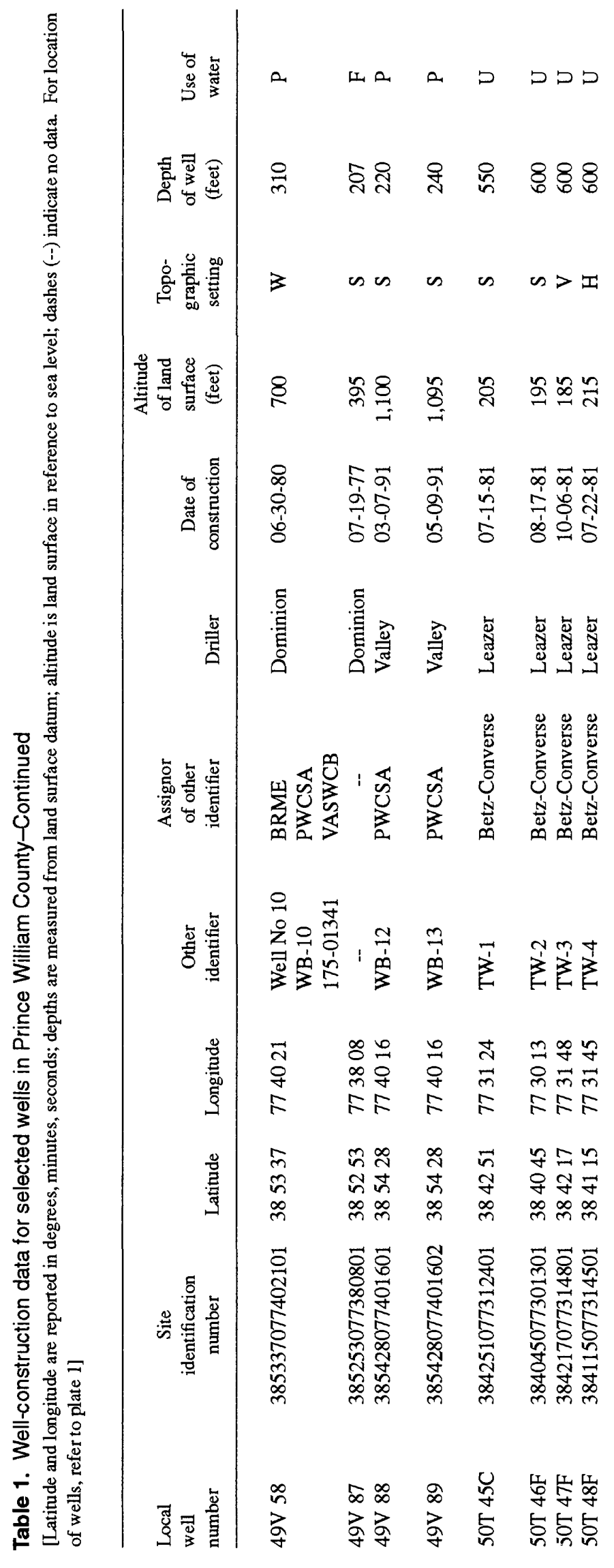




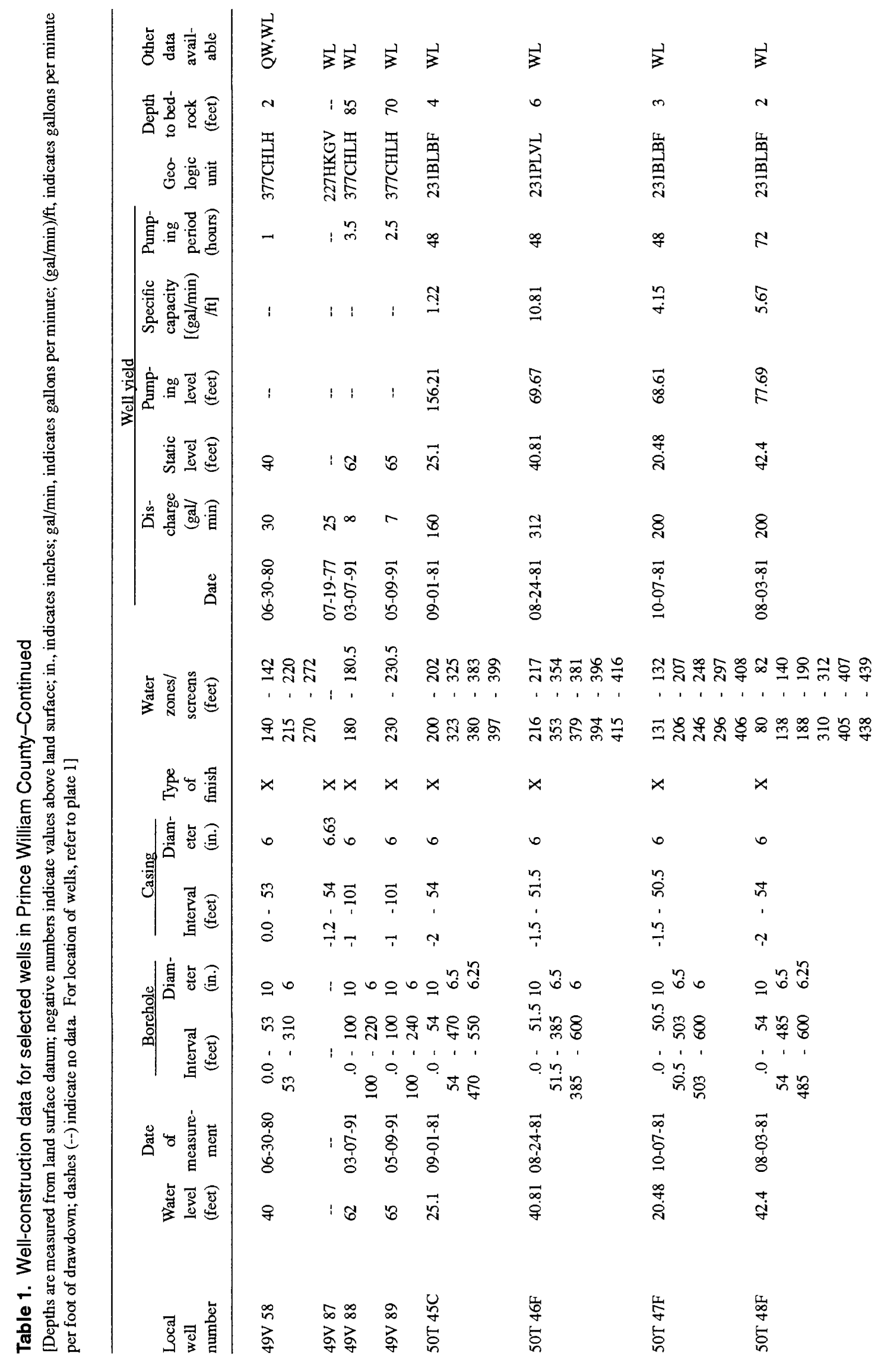




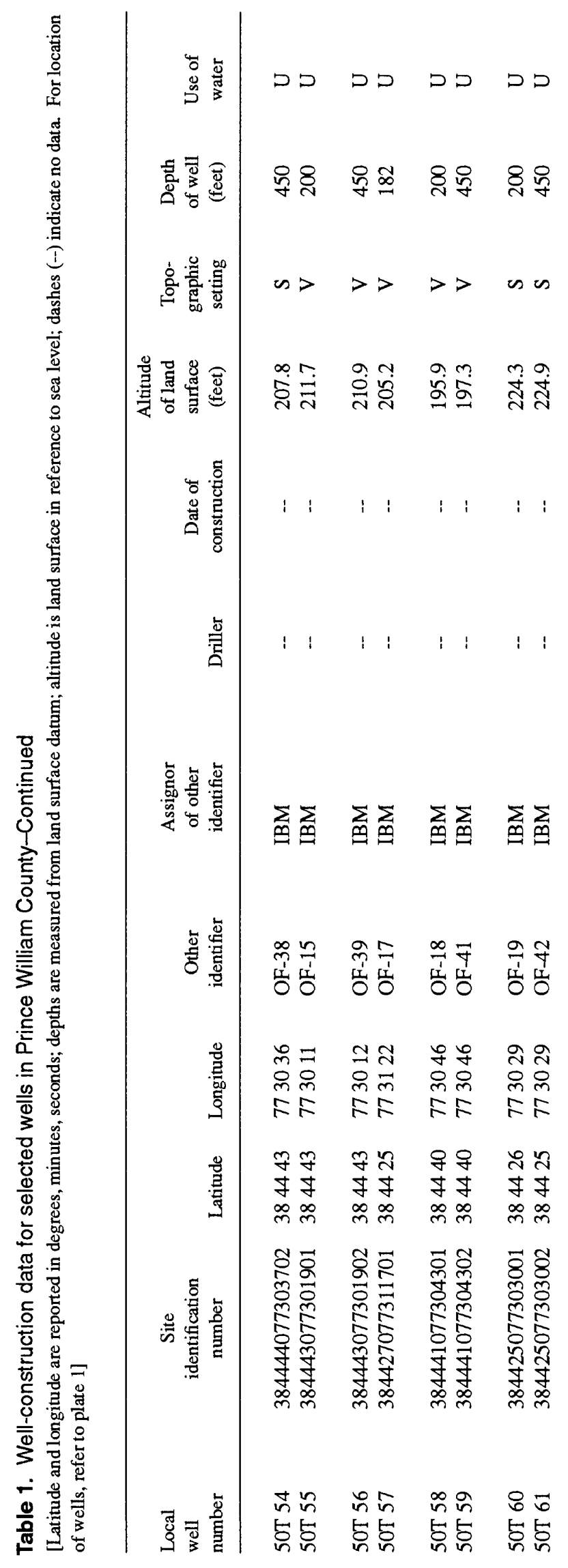




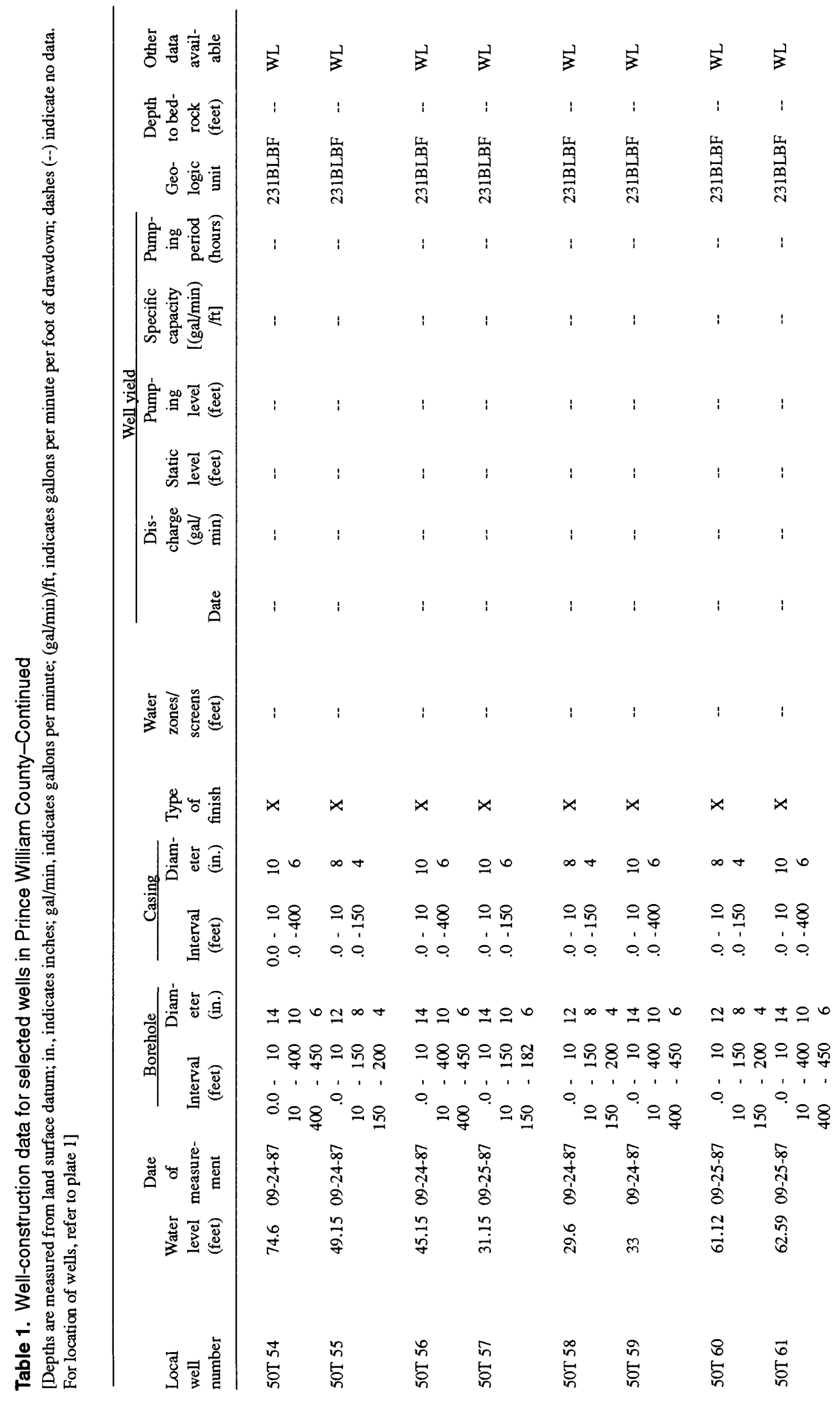




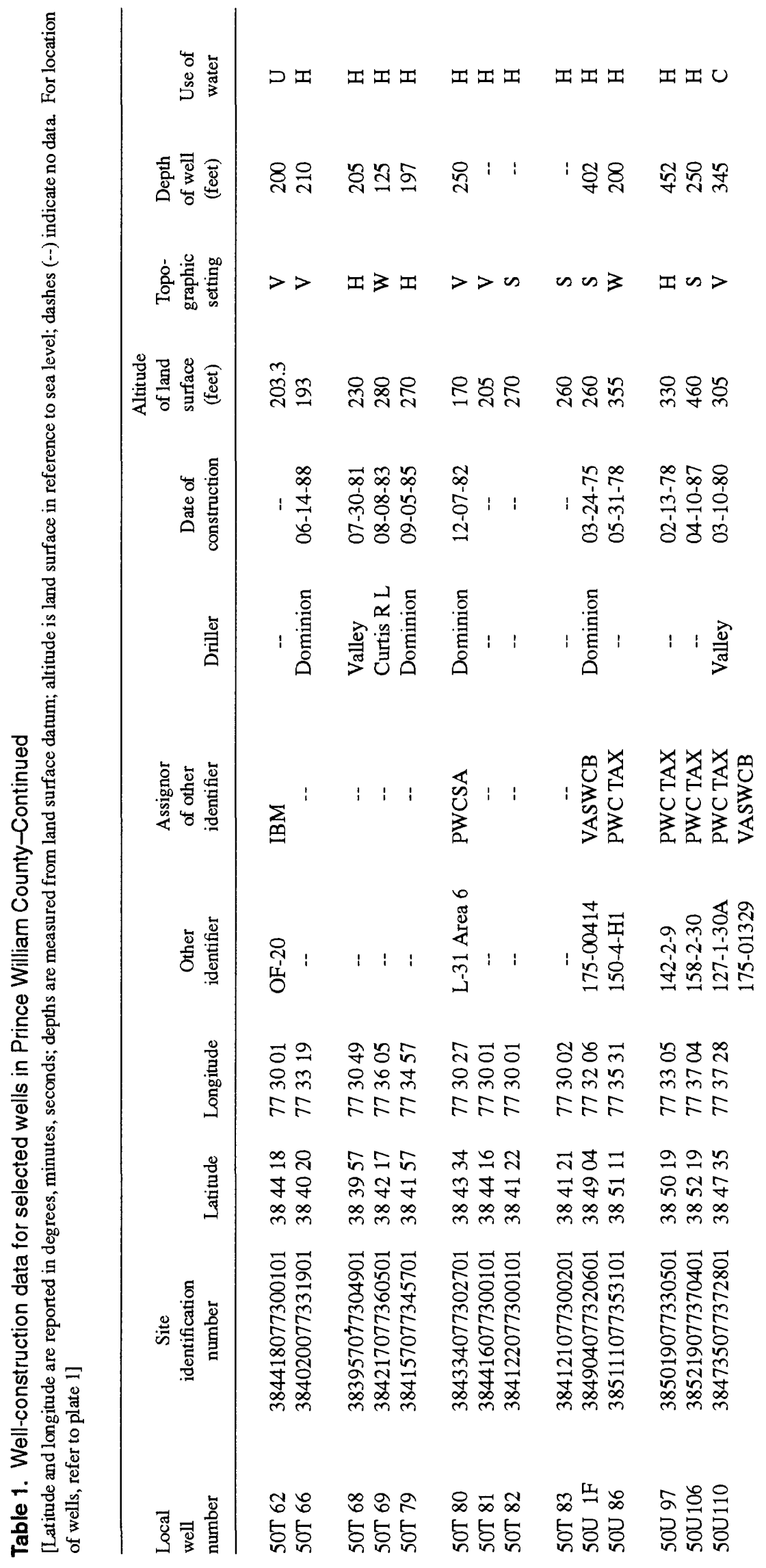




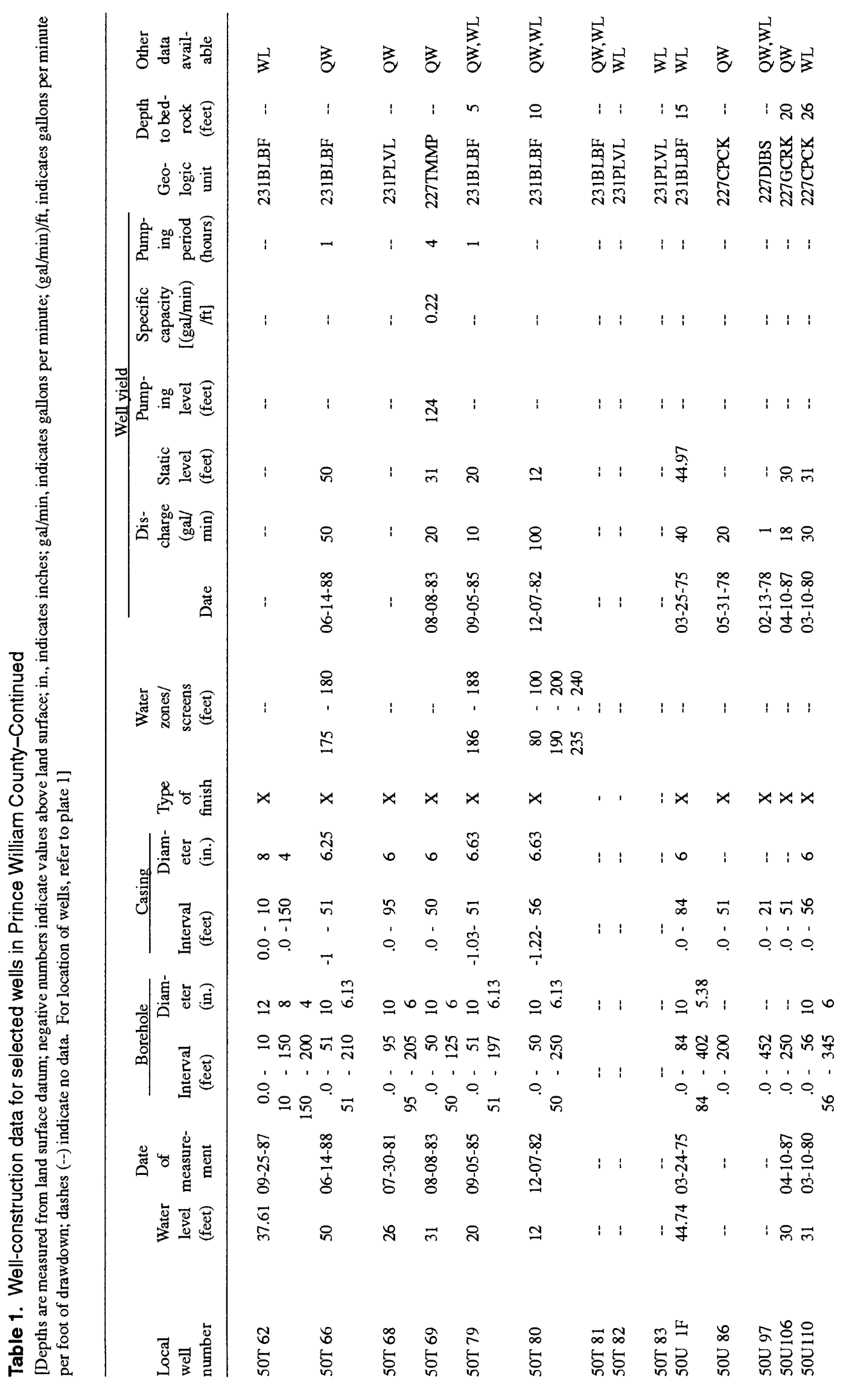




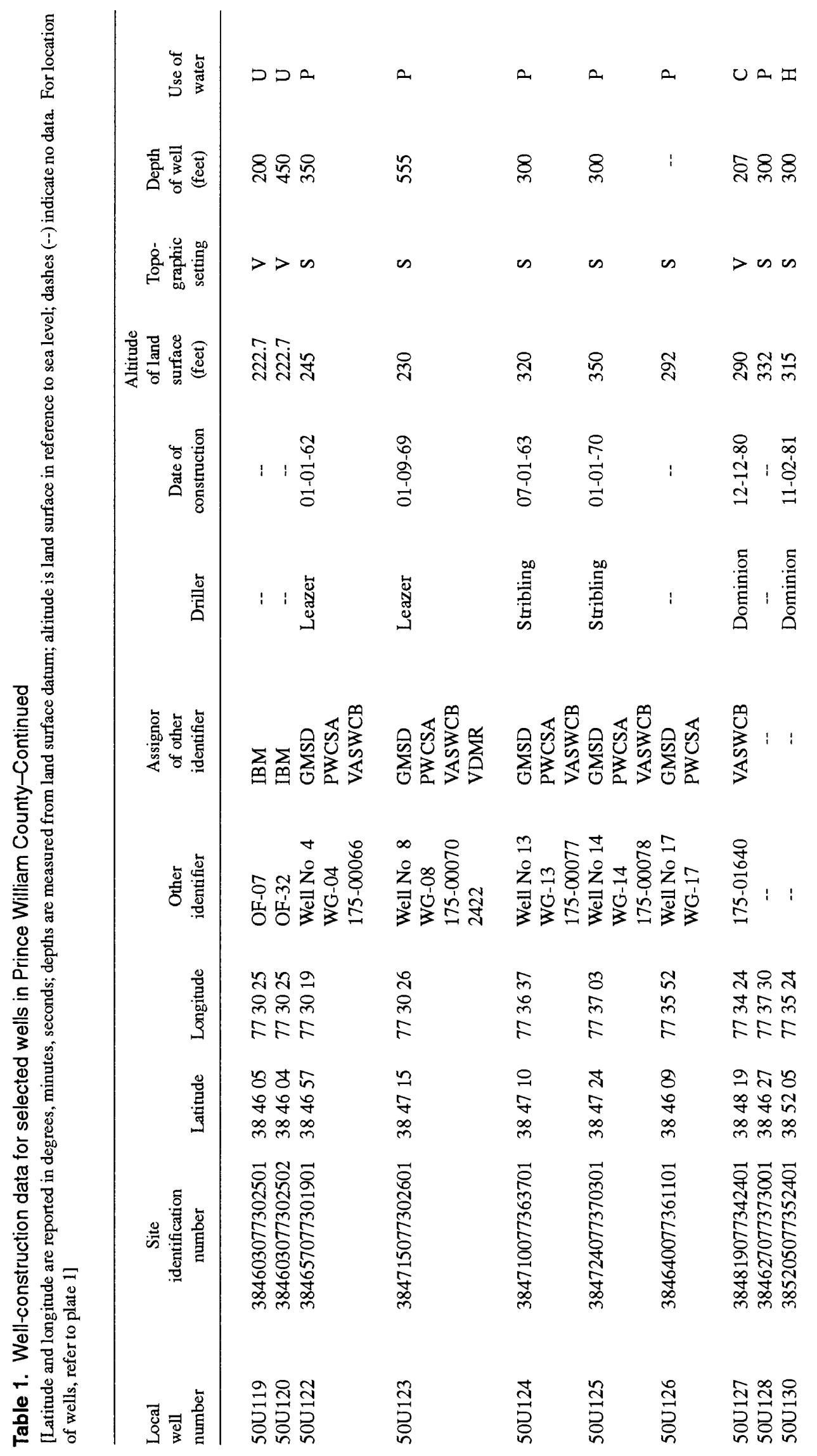




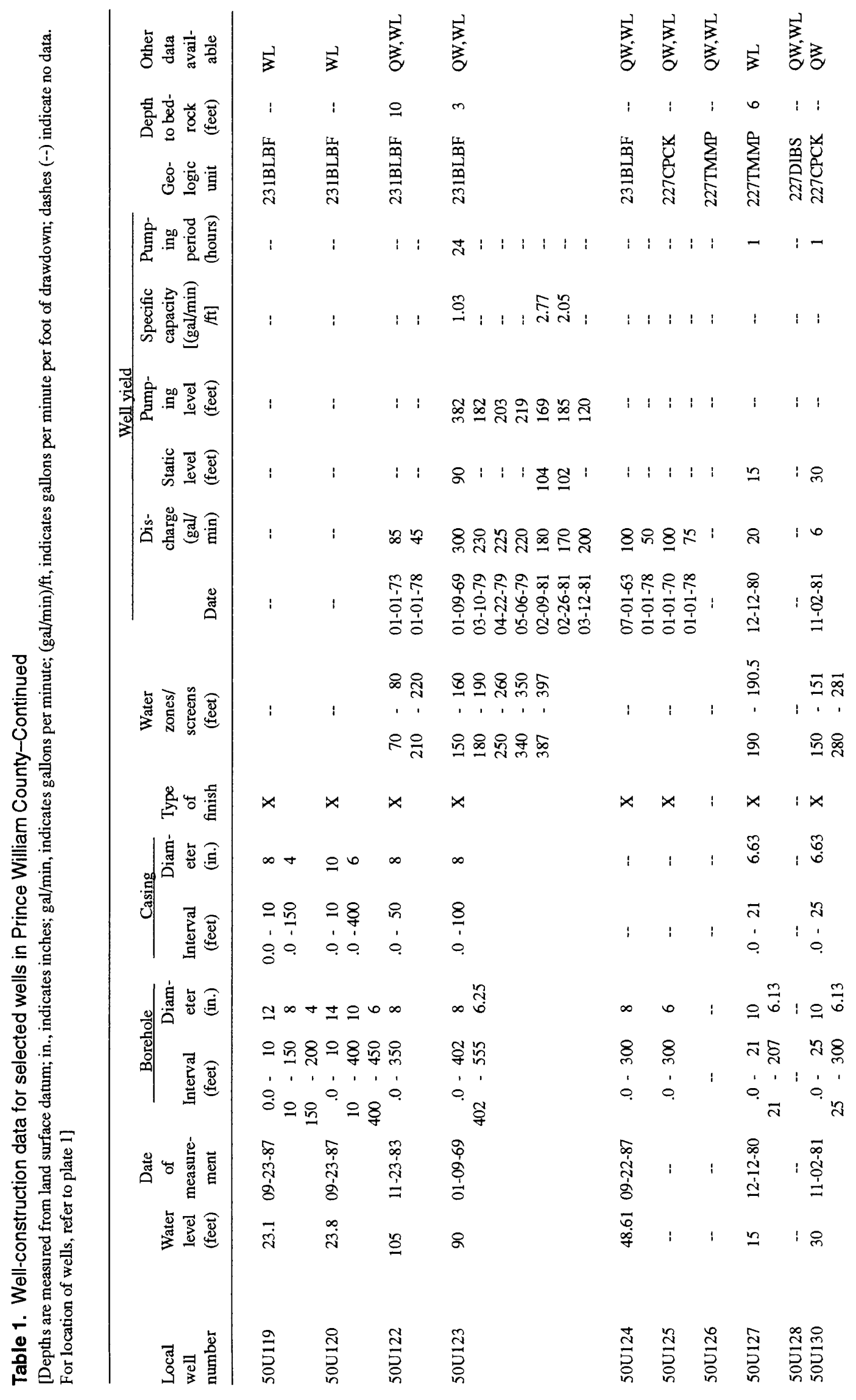




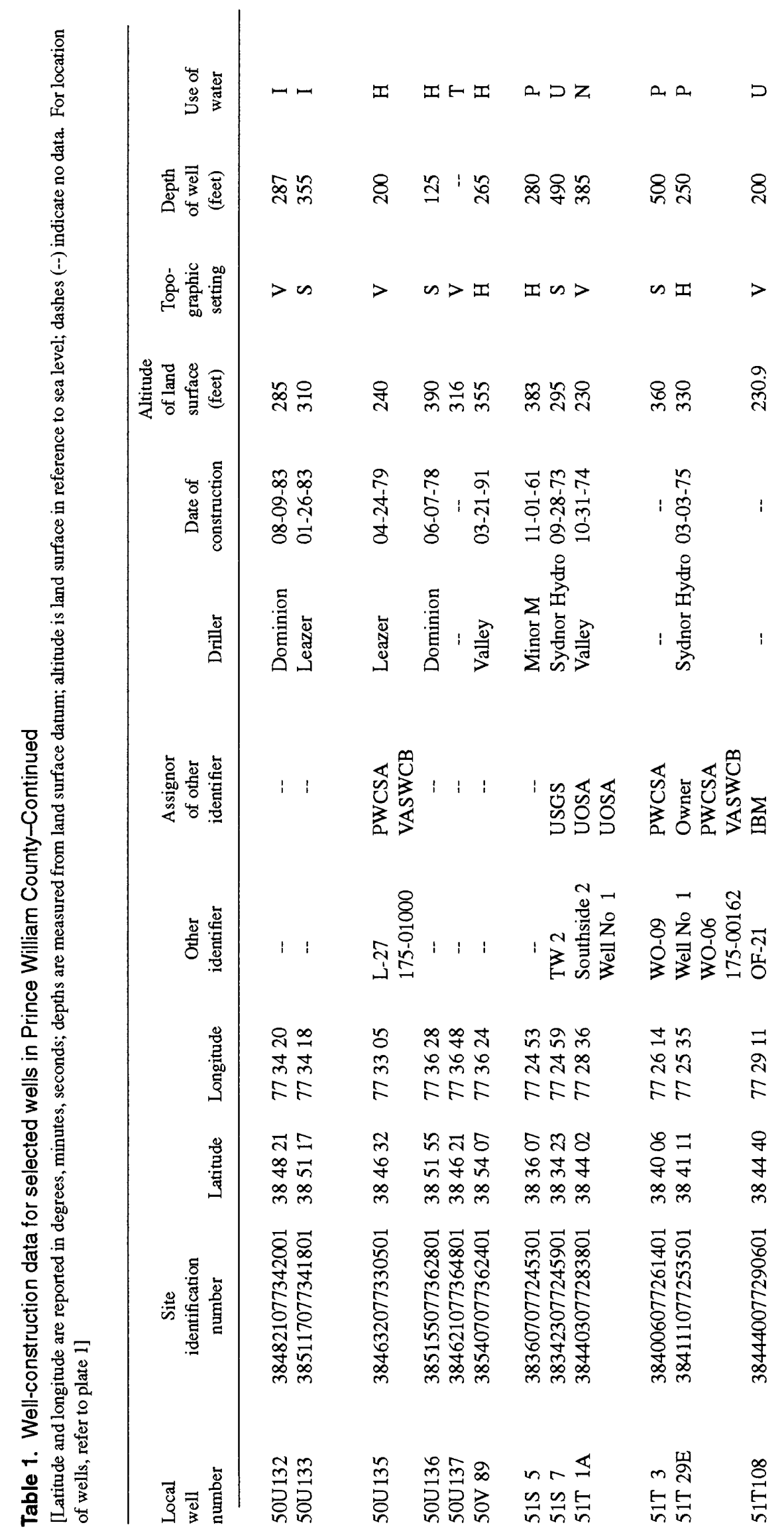




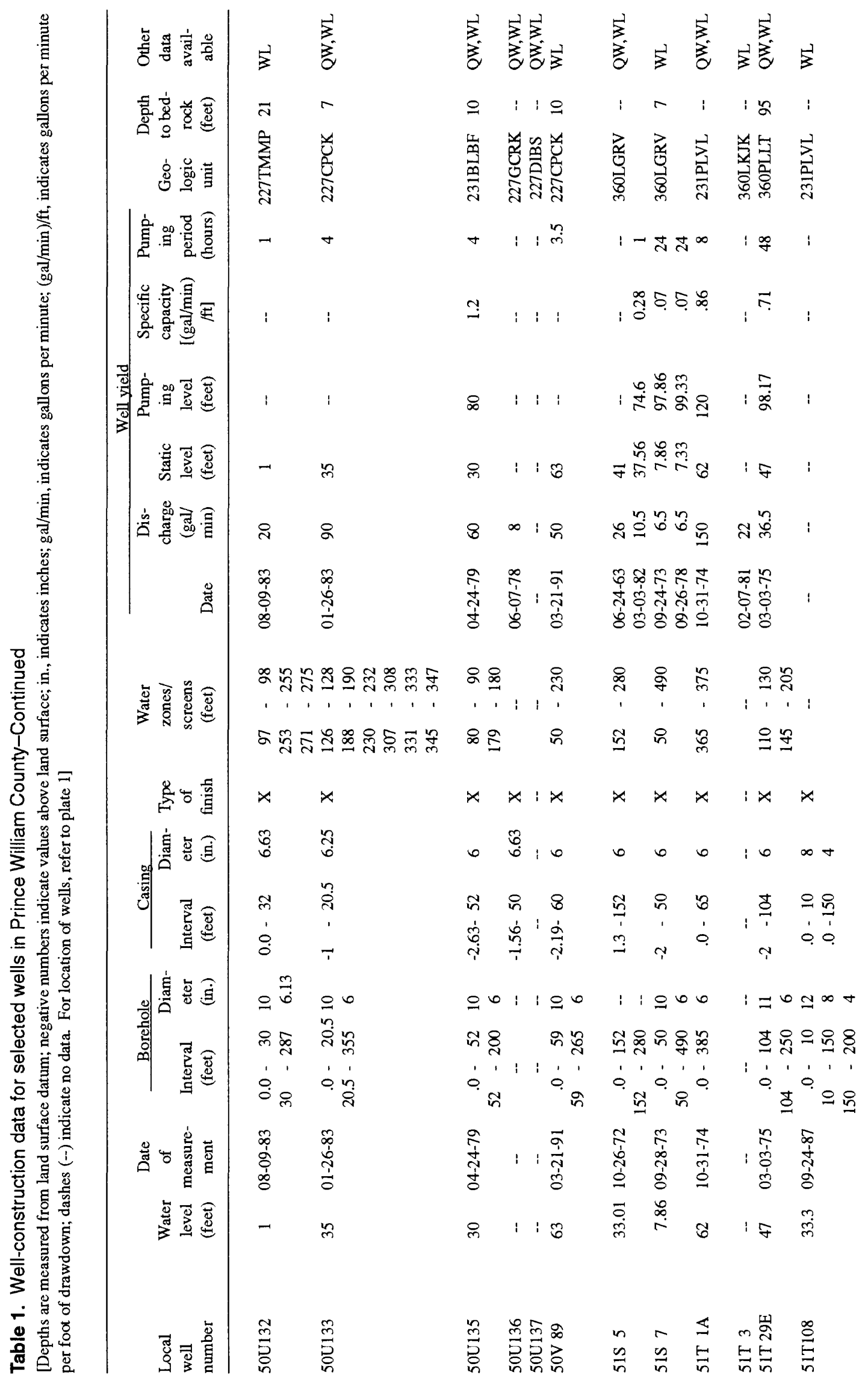




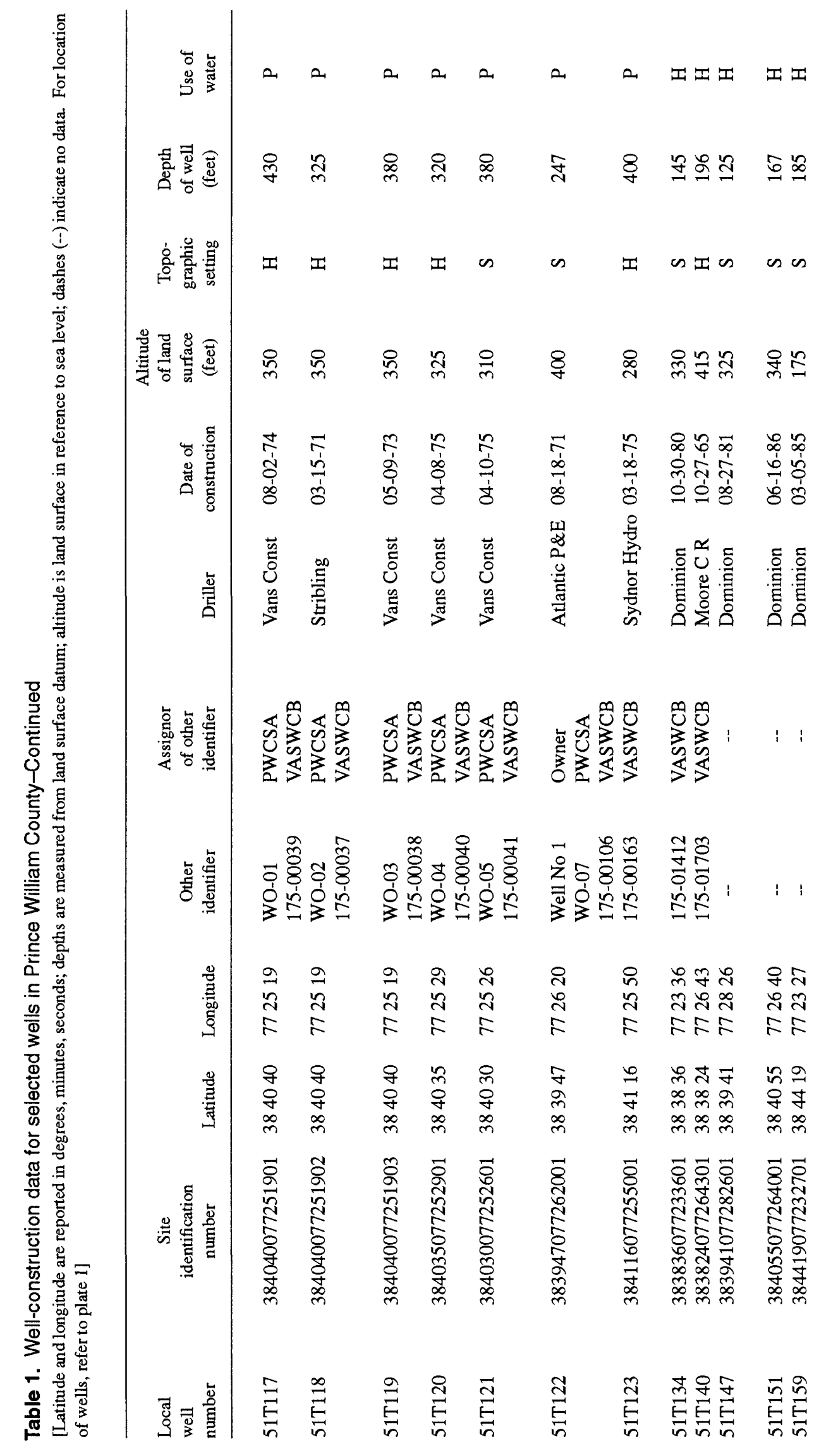




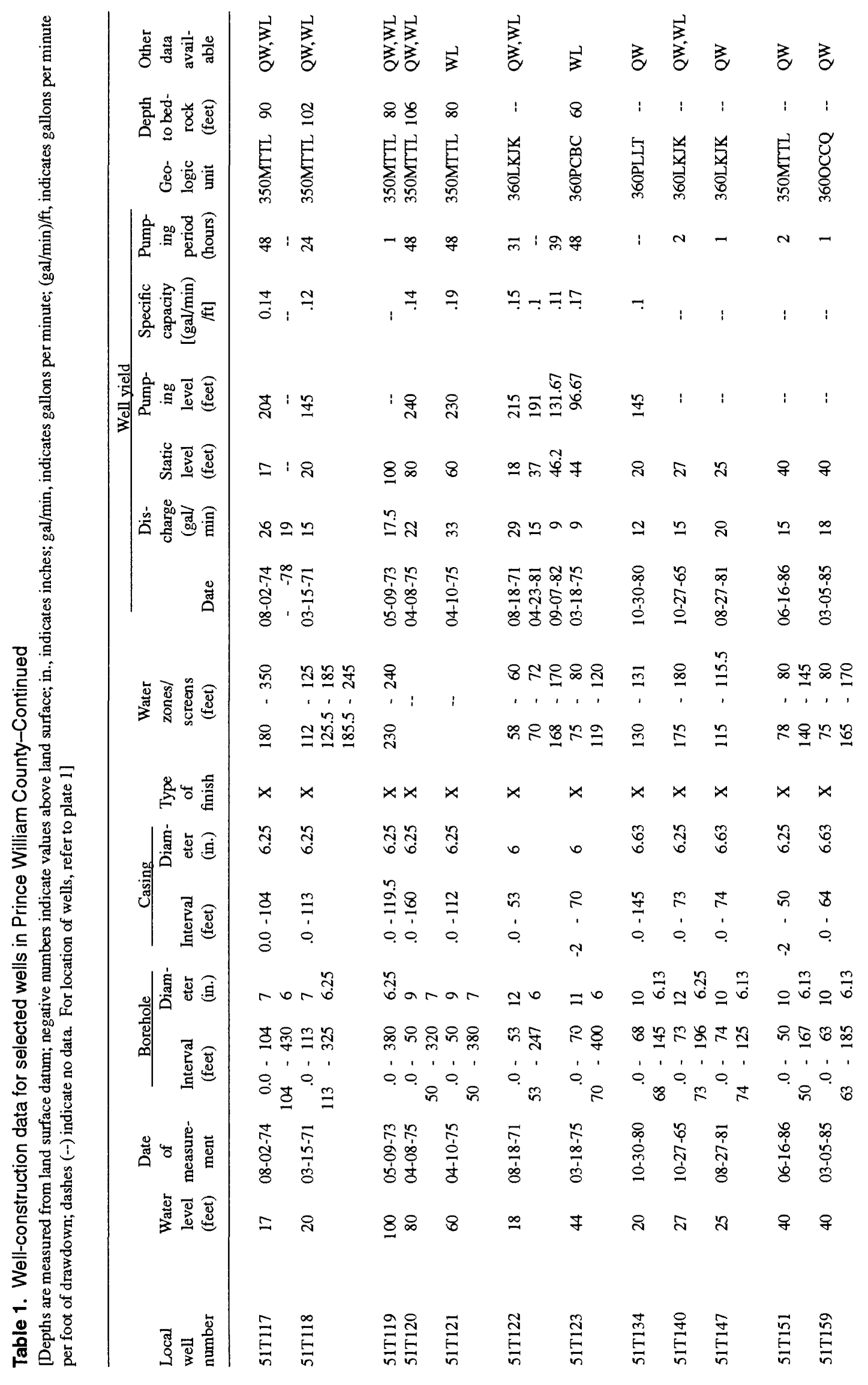




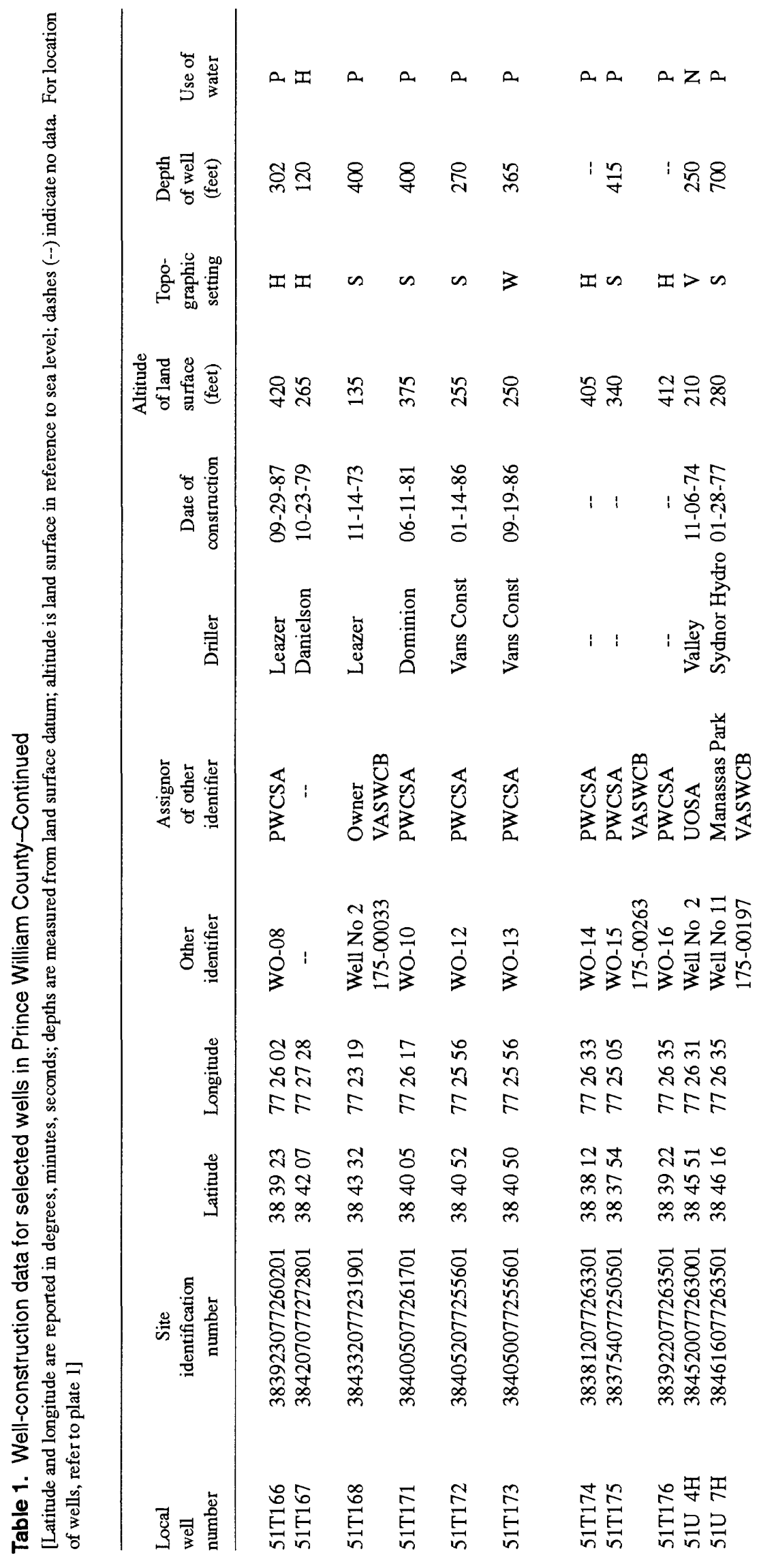




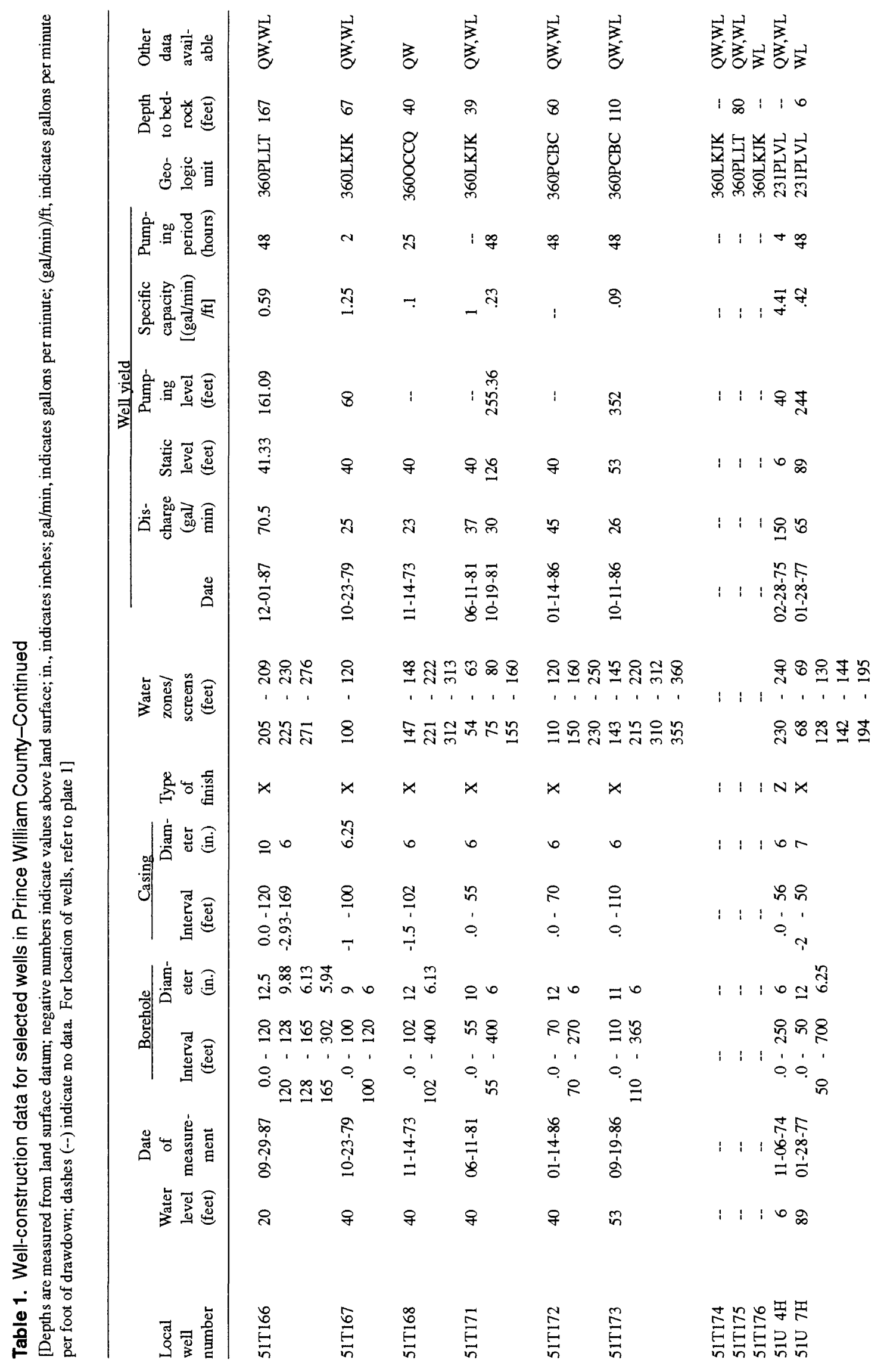




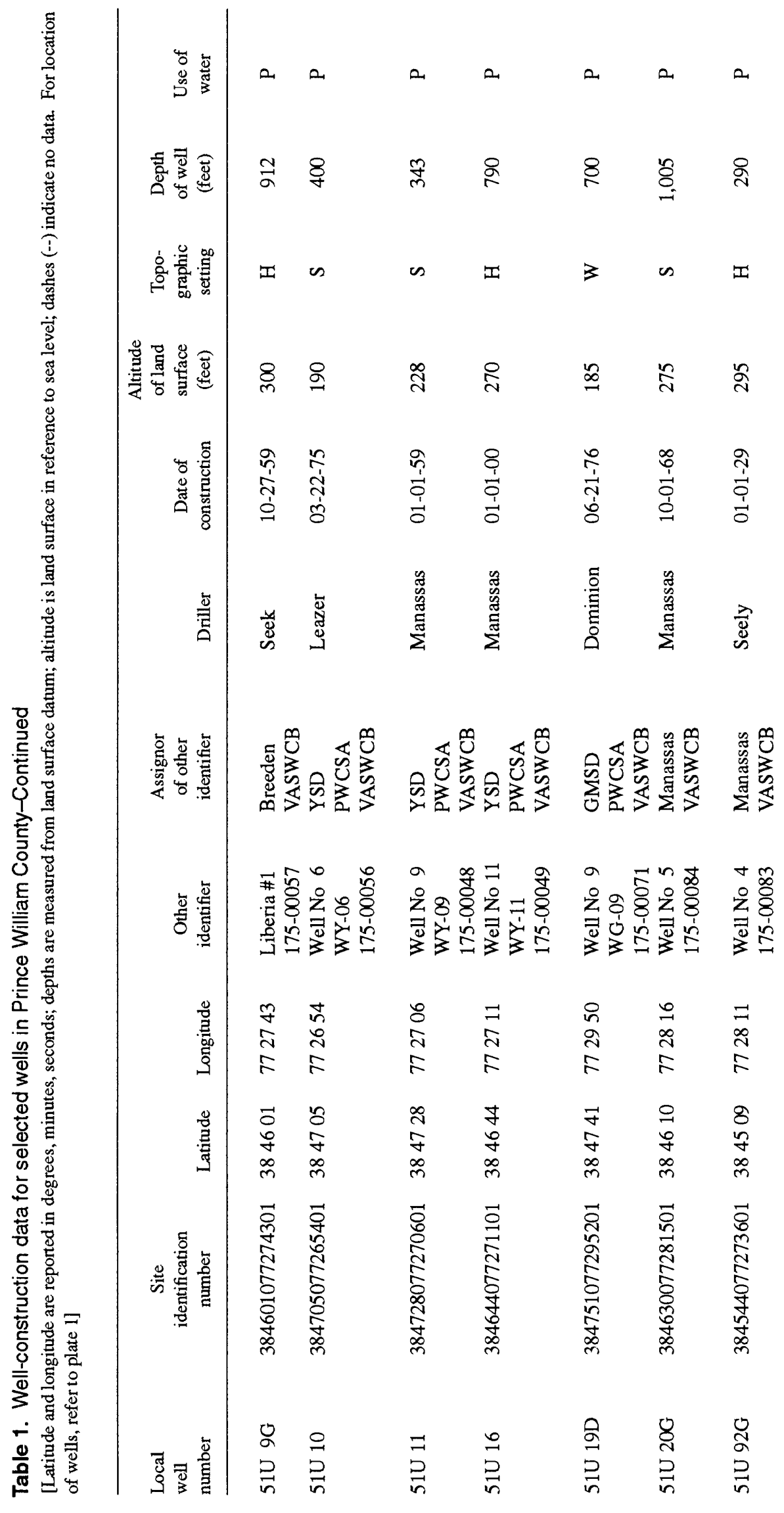




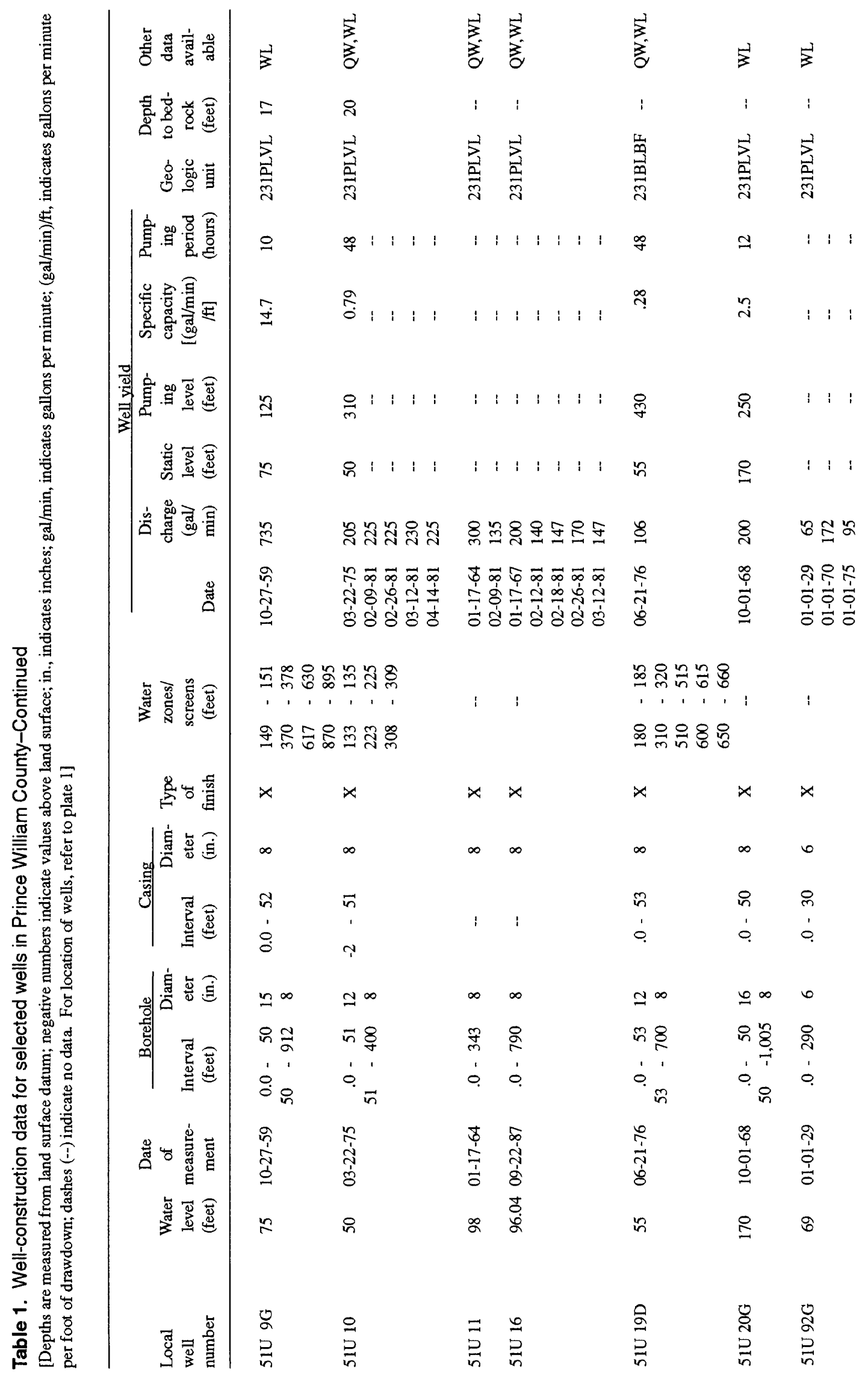




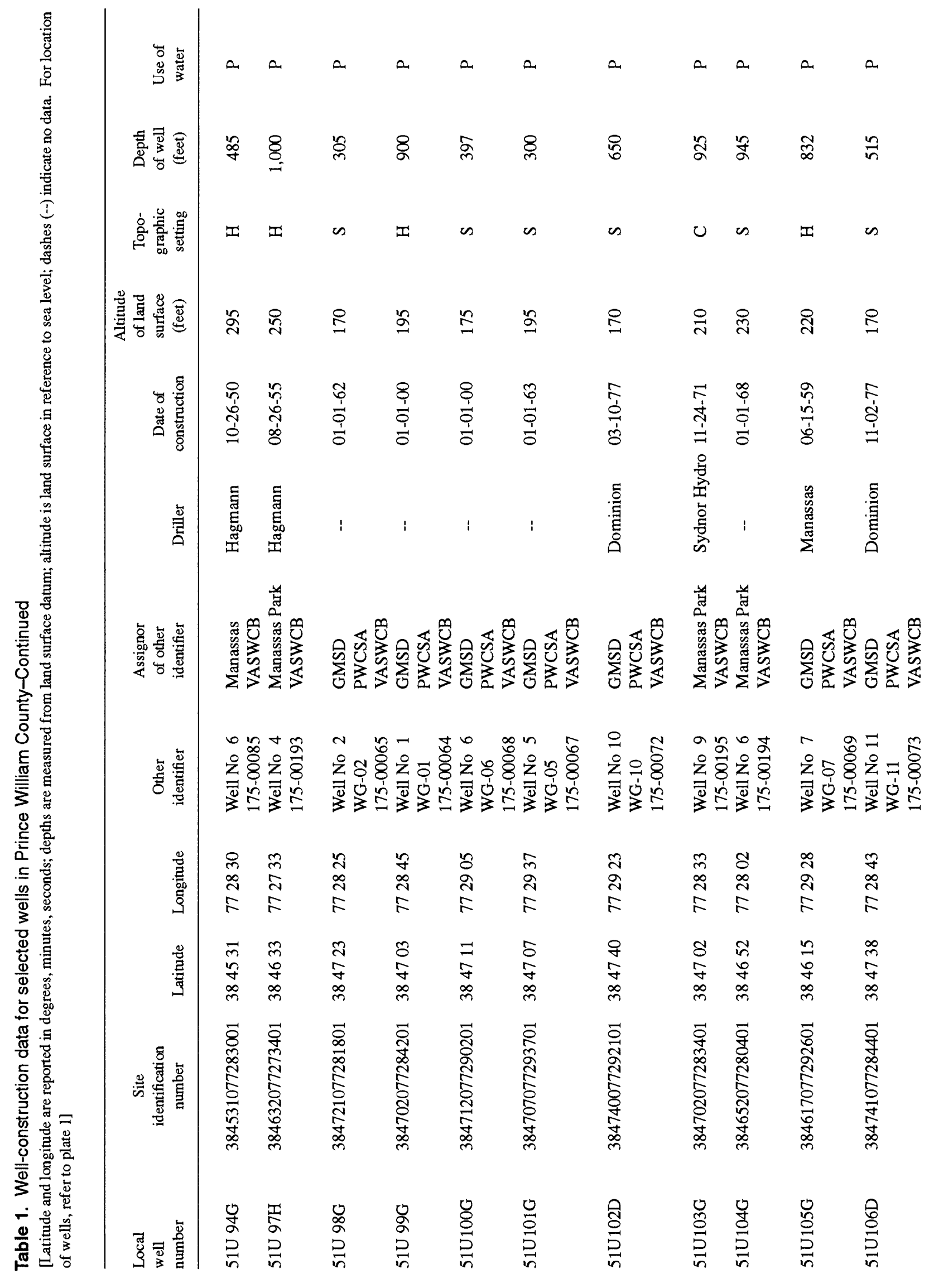




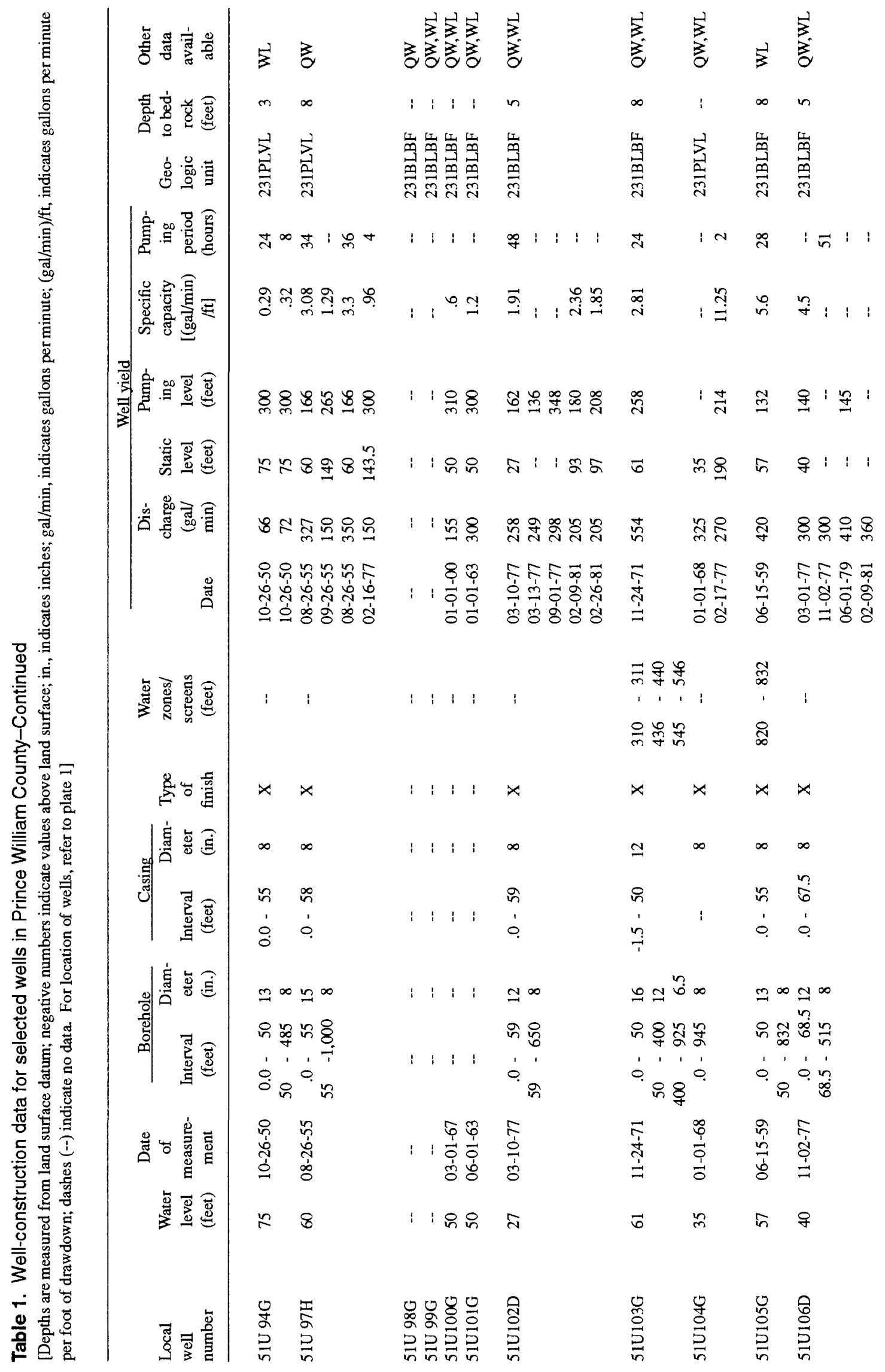




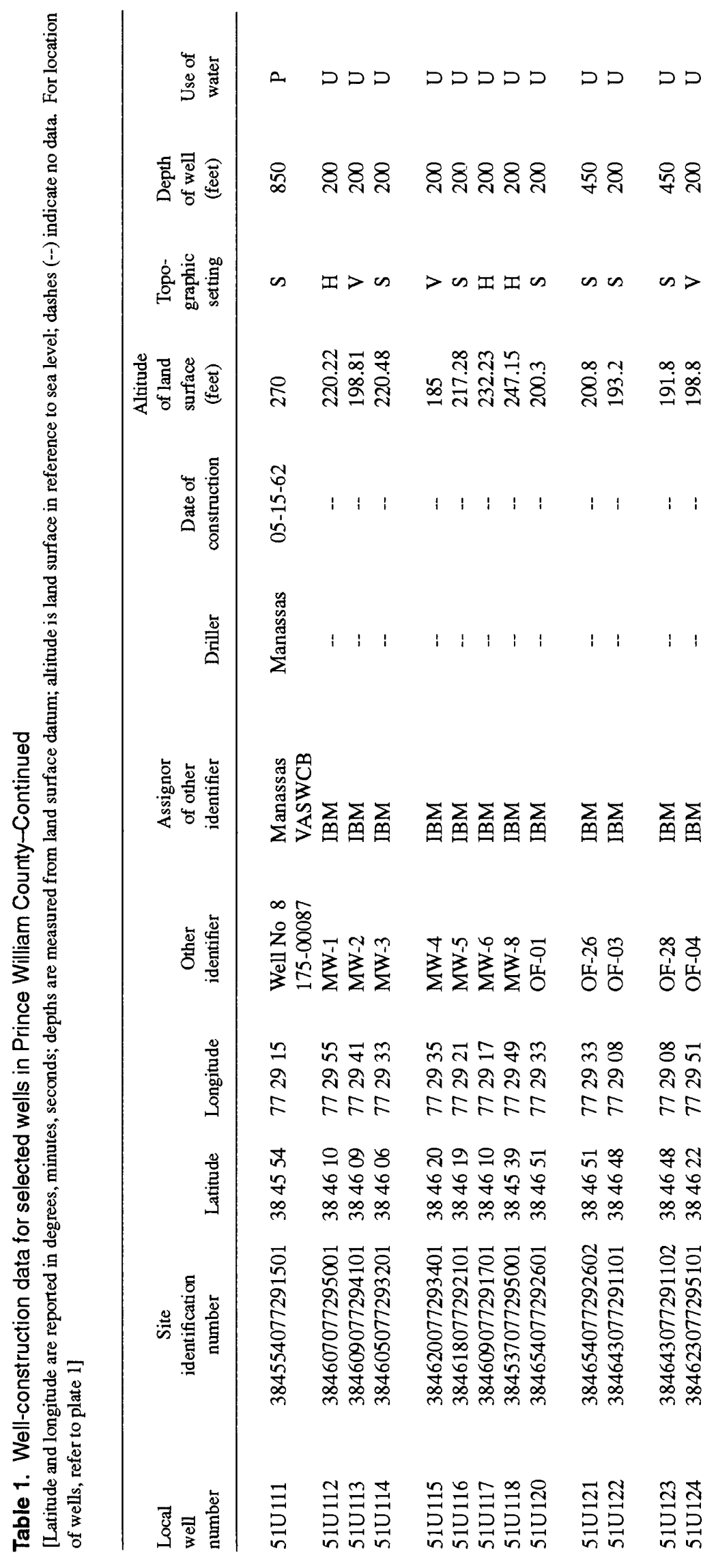




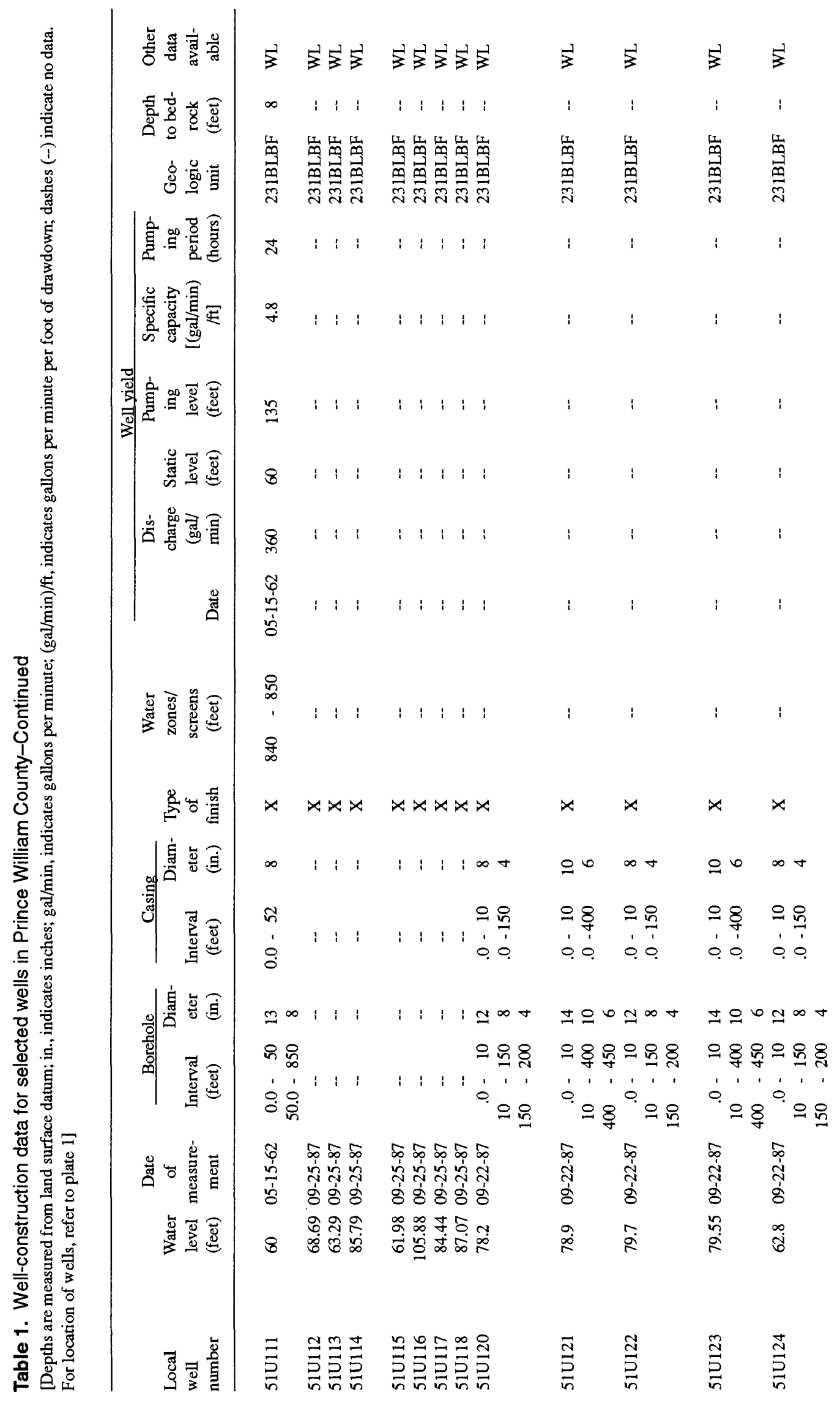




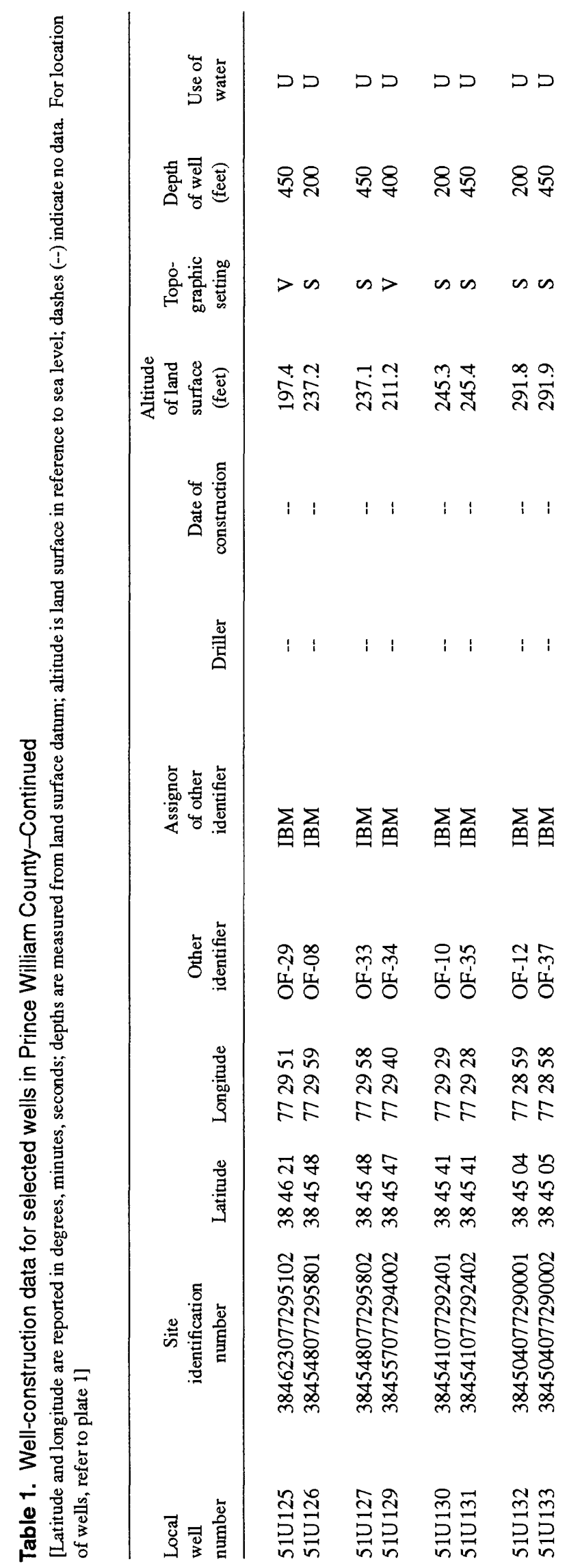




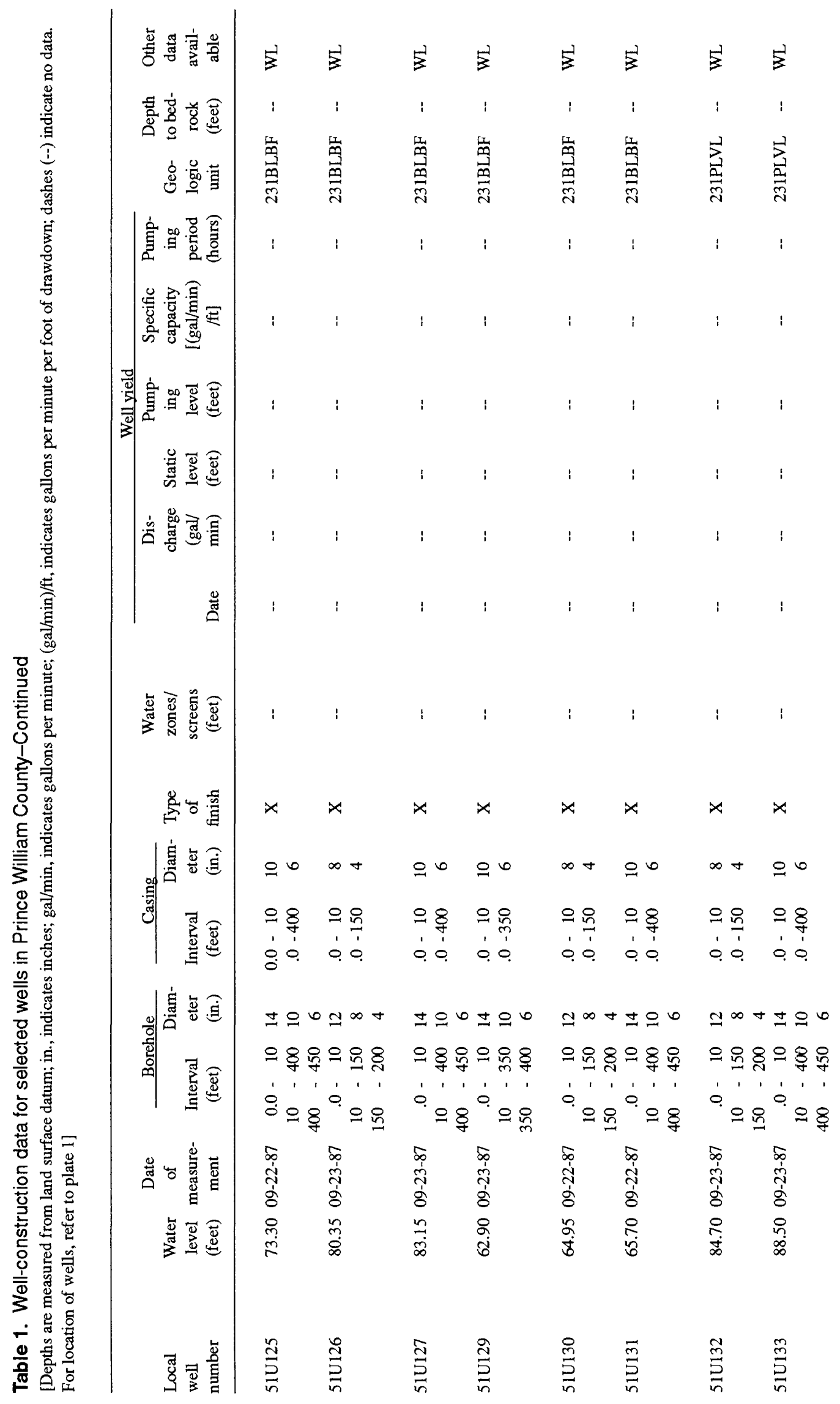




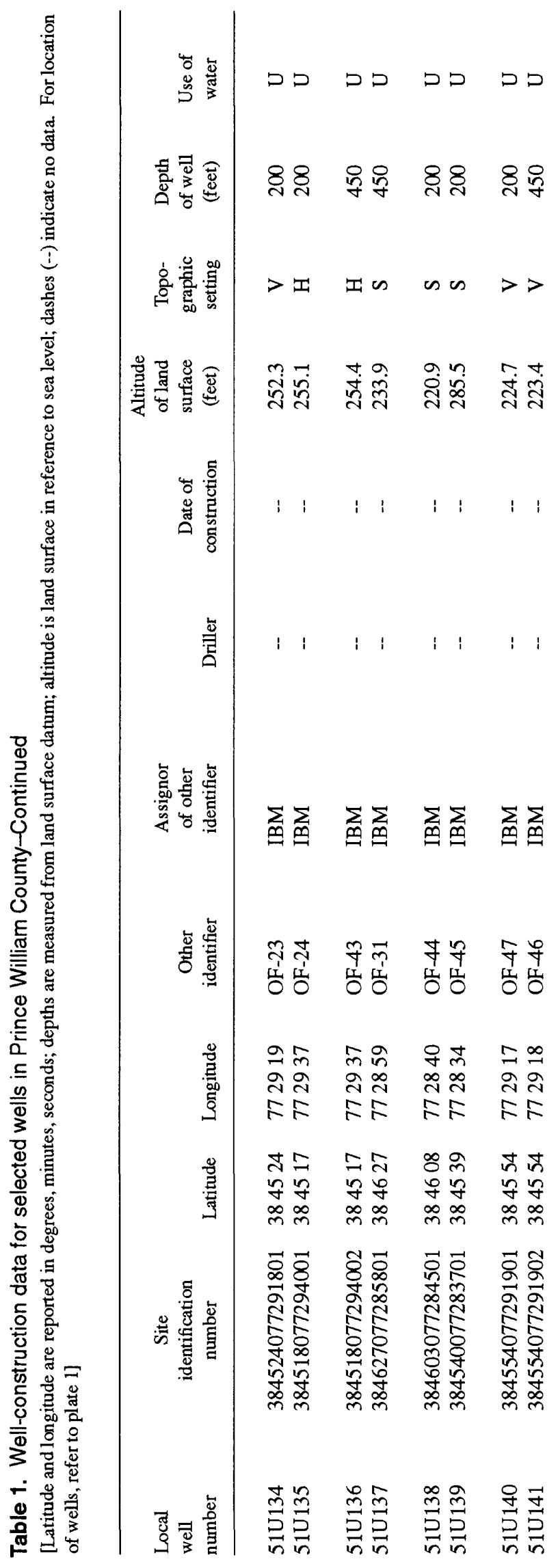




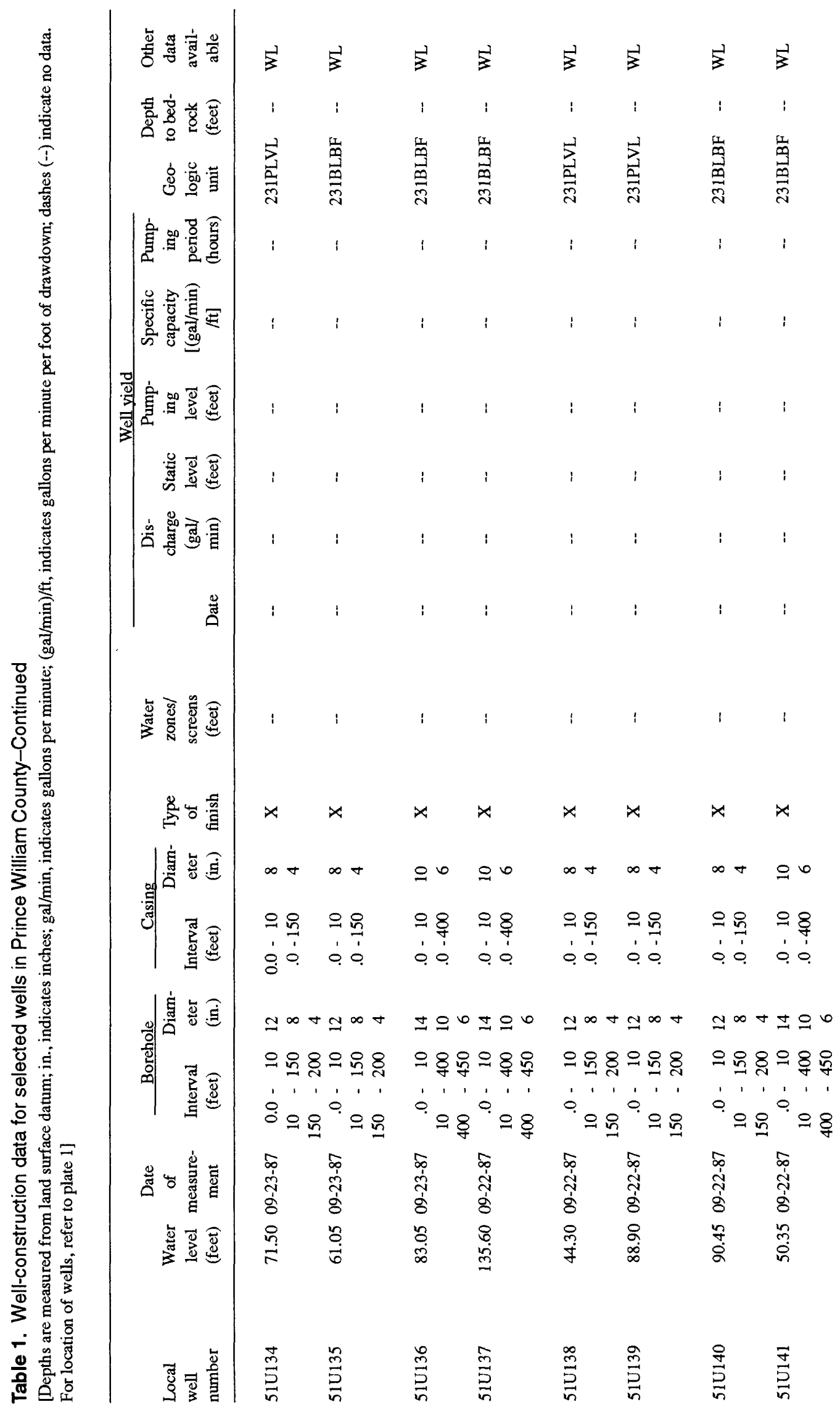




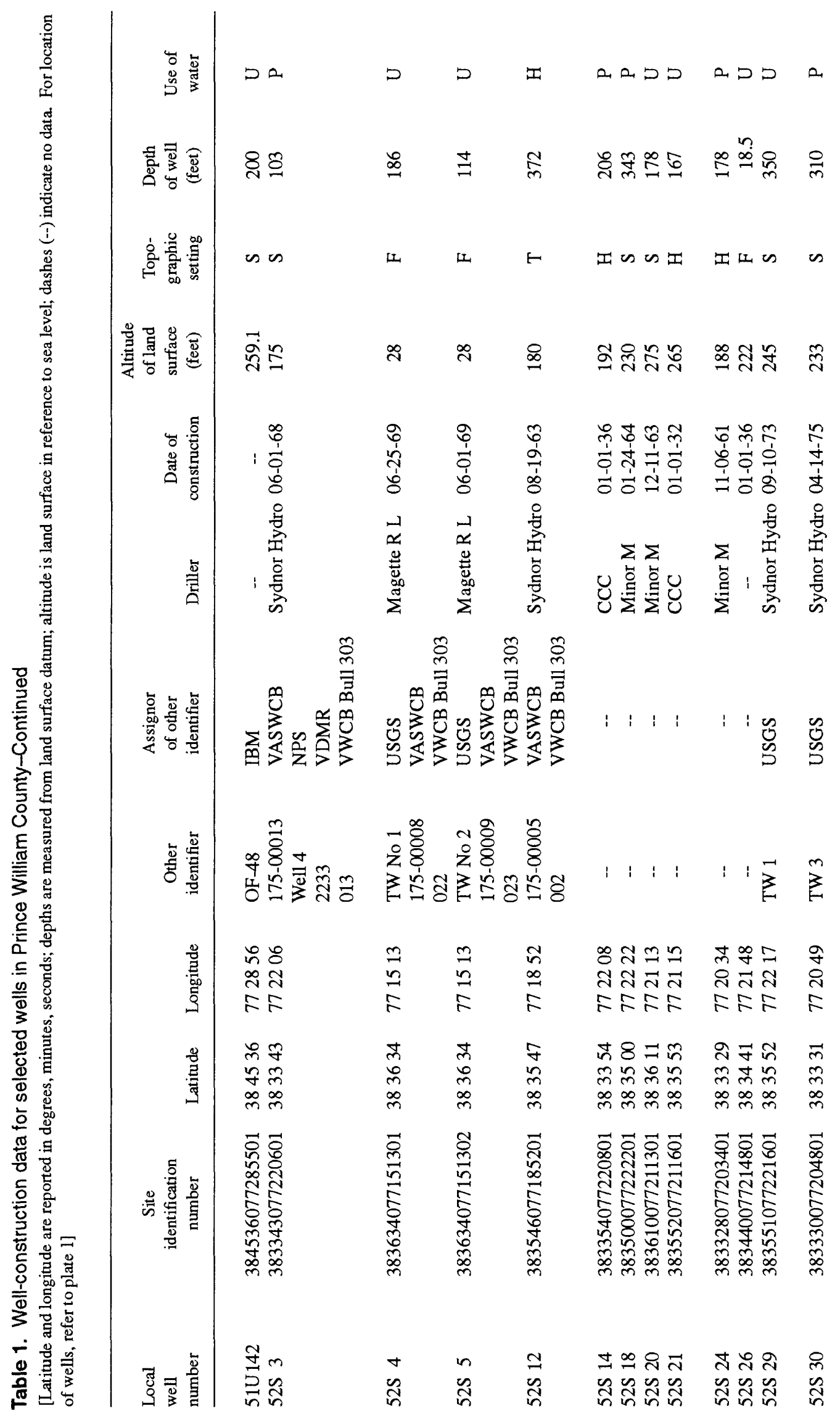




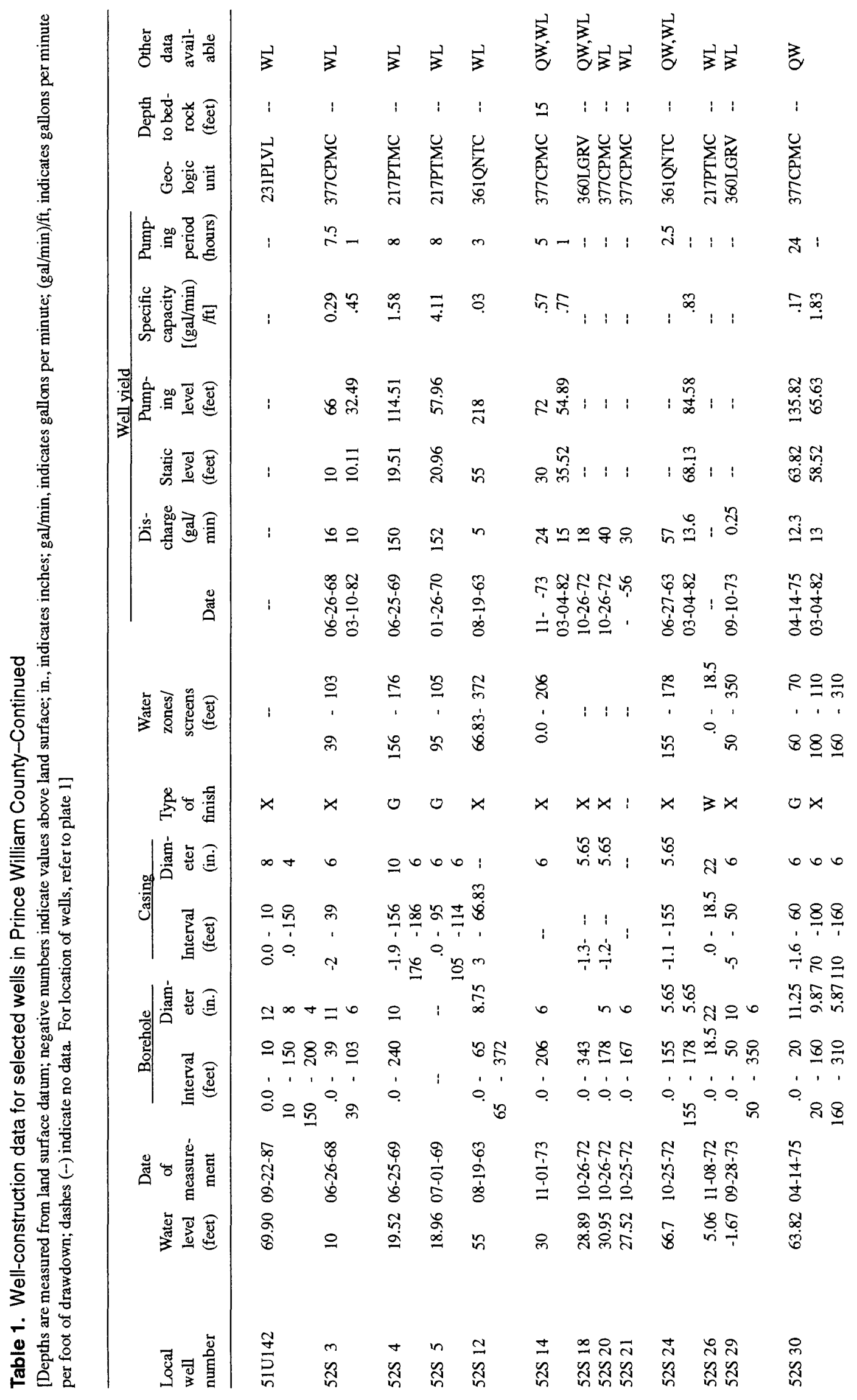




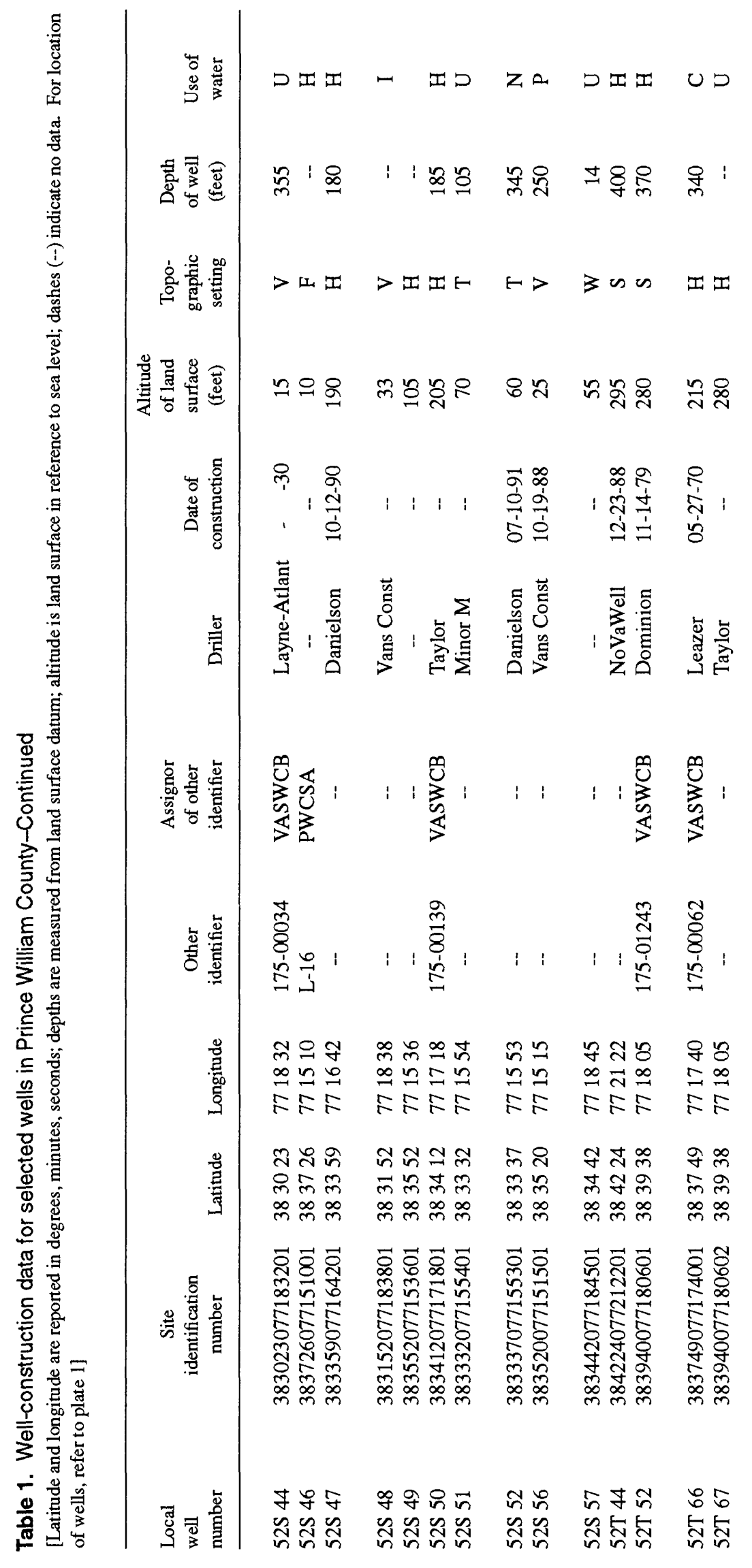




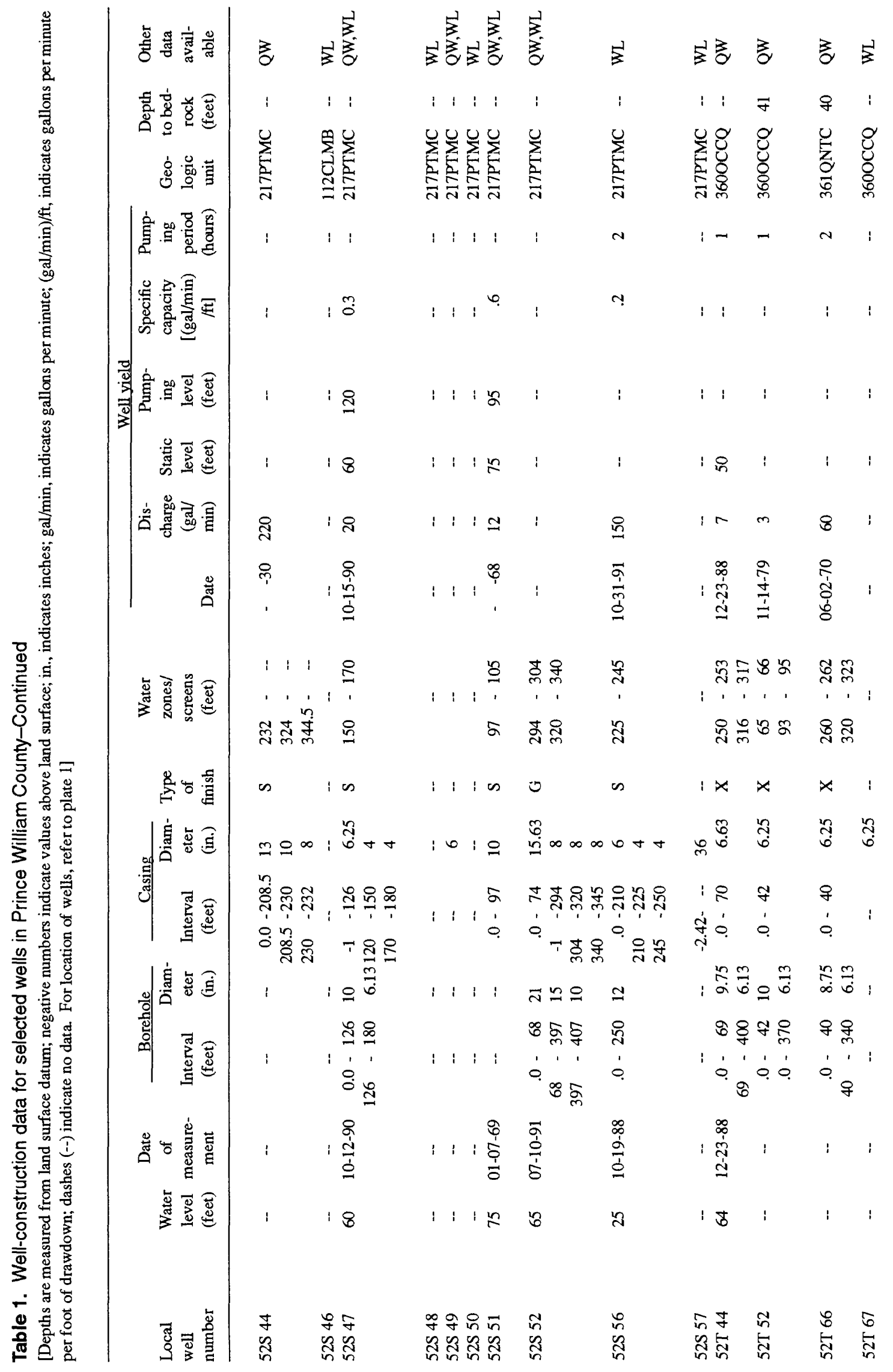




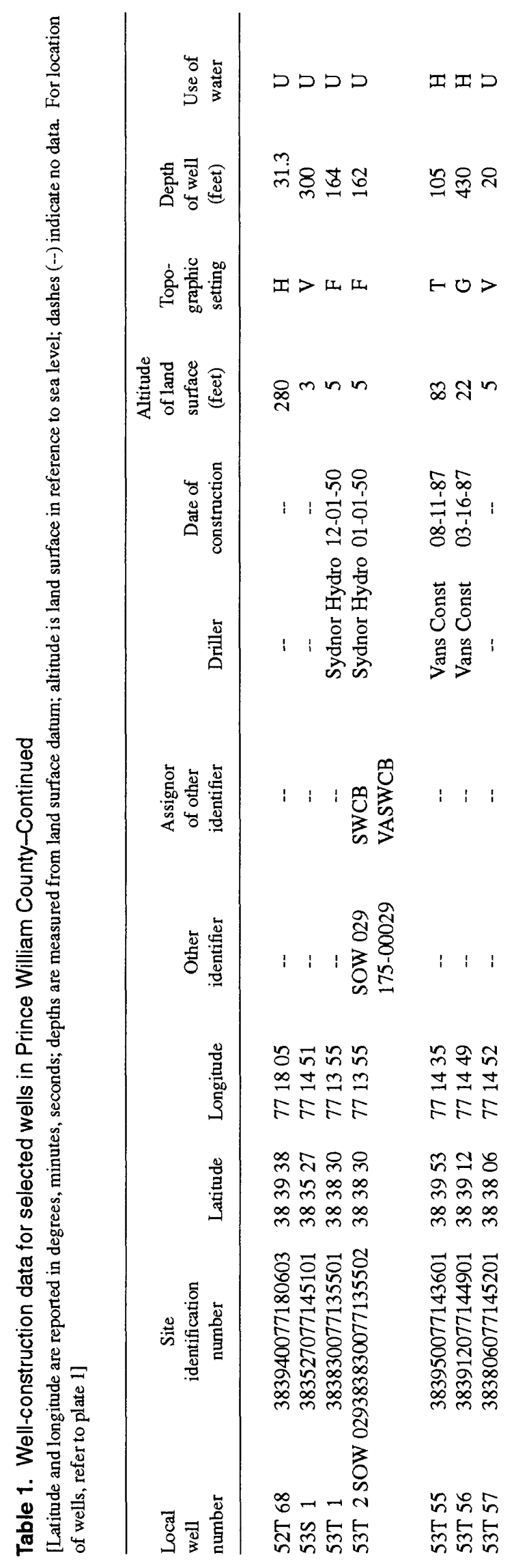




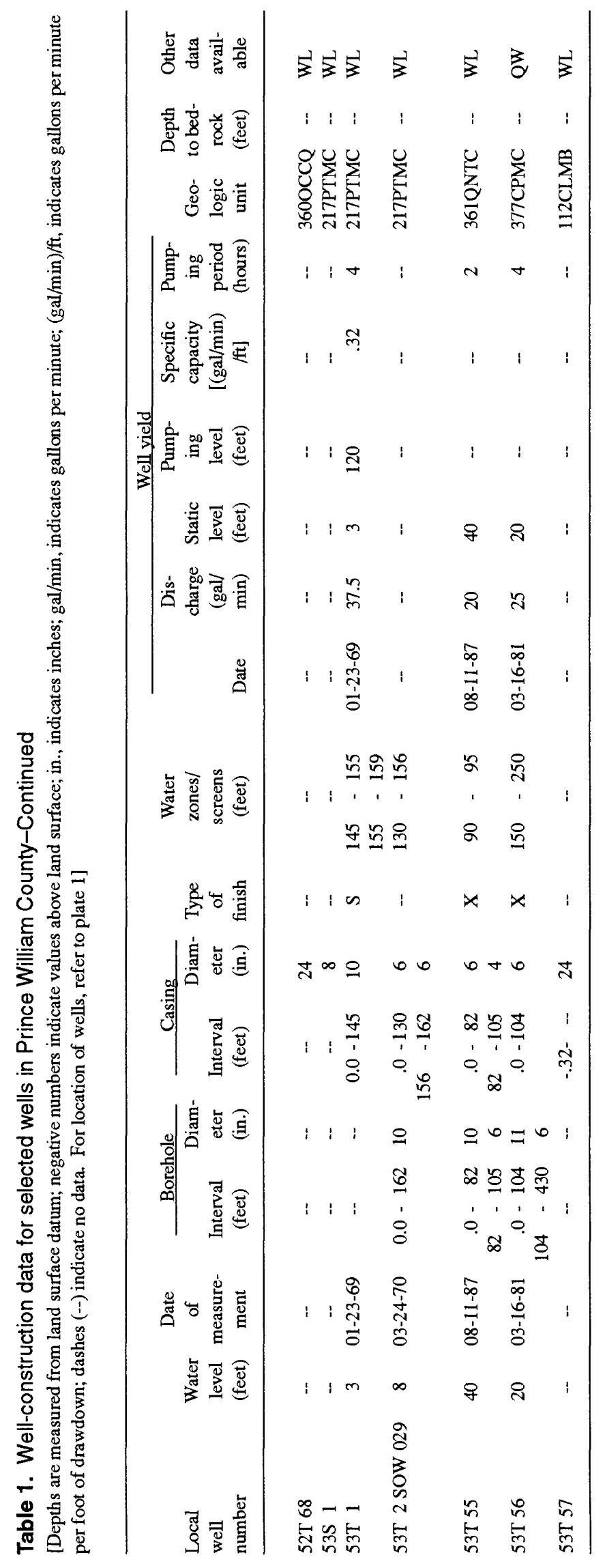


Table 2. Synoptic water-level measurements for selected wells in Prince William County

\section{EXPLANATION OF CODES}

$\begin{array}{ll} & \underline{\text { Agency }} \\ \text { IBM } & \text { International Business Machines, Inc } \\ \text { USGS } & \text { U.S. Geological Survey } \\ \text { VWCB } & \text { Virginia Water Control Board }\end{array}$

Method of measurement

$\begin{array}{ll}\text { C } & \text { Calibrated airline } \\ \text { G } & \text { Pressure gauge } \\ \text { S } & \text { Steel tape } \\ \text { T } & \text { Electric tape } \\ \text { X } & \text { Water level was not measured }\end{array}$

\section{$\underline{\text { Recovery period }}$}

N Unknown

U Unused well

W Observation well
Site status

$\begin{array}{ll}\text { D } & \text { Dry } \\ \text { F } & \text { Flowing } \\ \text { P } & \text { Pumped } \\ \text { R } & \text { Recently pumped } \\ \text { S } & \text { Static conditions }\end{array}$

D Dry

P Pumped

S Static conditions

\begin{tabular}{ll}
\multicolumn{2}{c}{ Geologic unit } \\
112CLMB & Columbia Group \\
217PTMC & Potomac Group \\
227CPCK & Catharpin Creek Formation of the Culpeper Group \\
227DIBS & Diabase \\
227GCRK & Goose Creek Member of the Catharpin Creek Formation \\
227HKGV & Hickory Grove Basalt of the Culpeper Group \\
227MDLD & Midland Formation of the Culpeper Group \\
227MZCC & Mount Zion Church Basalt of the Culpeper Group \\
227TMMP & Thermally metamorphosed rocks \\
227TRKR & Turkey Run Formation of the Culpeper Group \\
227WRFL & Waterfall Formation of the Culpeper Group \\
231BLBF & Balls Bluff Siltstone of the Culpeper Group \\
231PLVL & Poolesville Member of the Manassas Sandstone \\
377CPMC & Chopawamsic Formation \\
350MTTL & Metatonalite 1 \\
360LGRV & Lunga Reservoir Formation \\
360LKJK & Lake Jackson Pluton \\
360OCCQ & Occoquan Pluton \\
360PCBC & Purcell Branch Formation \\
360PLLT & Phyllite 1 \\
361QNTC & Quantico Formation \\
377CHLH & Chilhowee Group
\end{tabular}

${ }^{1}$ Unit follows usage of U.S. Geological Survey Open-File Report 90-548 (Pavlides, 1990). 
Table 2. Synoptic water-level measurements for selected wells in Prince William County-Continued

[Depths are measured from land surface datum; negative numbers indicate values above land surface; dashes (-) indicate that water levels were not measured. For location of wells, refer to plate 1]

\begin{tabular}{|c|c|c|c|c|c|c|c|}
\hline $\begin{array}{c}\text { Local } \\
\text { well } \\
\text { number }\end{array}$ & $\begin{array}{l}\text { Geologic } \\
\text { unit }\end{array}$ & Date & Agency & $\begin{array}{c}\text { Recovery } \\
\text { period } \\
\text { (hours) }\end{array}$ & $\begin{array}{l}\text { Water } \\
\text { level } \\
\text { (feet) }\end{array}$ & $\begin{array}{l}\text { Site } \\
\text { status }\end{array}$ & $\begin{array}{l}\text { Method of } \\
\text { measurment }\end{array}$ \\
\hline \multirow[t]{2}{*}{$49 \mathrm{~T} 74$} & 231BLBF & $03-27-91$ & USGS & $\mathrm{N}$ & 30.44 & $\mathrm{R}$ & S \\
\hline & & $10-24-91$ & USGS & $\mathrm{N}$ & 37.02 & $\mathrm{~S}$ & $\mathrm{~S}$ \\
\hline \multirow[t]{2}{*}{$49 \mathrm{U} 1$} & 227WRFL & $04-26-91$ & USGS & W & 5.05 & $\mathrm{~S}$ & $\mathrm{~S}$ \\
\hline & & $10-29-91$ & USGS & W & 10.01 & $S$ & $S$ \\
\hline \multirow[t]{2}{*}{$49 \mathrm{U} 60$} & $227 \mathrm{CPCK}$ & $03-28-91$ & USGS & $\mathrm{N}$ & 27.42 & $\mathrm{R}$ & $S$ \\
\hline & & $10-23-91$ & USGS & $\mathrm{N}$ & 31.12 & $\mathrm{R}$ & $S$ \\
\hline \multirow[t]{2}{*}{$49 \mathrm{U} 62$} & $227 \mathrm{MZCC}$ & $03-21-91$ & USGS & $\mathrm{N}$ & 22.32 & $\mathrm{R}$ & $S$ \\
\hline & & $10-17-91$ & USGS & 0.5 & 39.30 & $\mathrm{R}$ & $S$ \\
\hline \multirow[t]{2}{*}{$49 \cup 72$} & $227 \mathrm{CPCK}$ & $03-21-91$ & USGS & 1.3 & 75.97 & $\mathrm{R}$ & $S$ \\
\hline & & $10-22-91$ & USGS & 11.3 & 55.92 & $\mathrm{R}$ & $S$ \\
\hline \multirow[t]{2}{*}{$49 \mathrm{U} 73$} & $227 \mathrm{CPCK}$ & $03-21-91$ & USGS & .8 & 51.59 & $\mathrm{R}$ & $S$ \\
\hline & & $10-22-91$ & USGS & 1.3 & 64.25 & $\mathrm{R}$ & $S$ \\
\hline \multirow[t]{2}{*}{$49 \mathrm{U} 74$} & $227 \mathrm{MZCC}$ & $04-02-91$ & USGS & $\mathrm{N}$ & 31.27 & $\mathrm{R}$ & S \\
\hline & & $10-23-91$ & USGS & 16 & 33.63 & $S$ & $S$ \\
\hline \multirow[t]{2}{*}{$49 U 75$} & 227TMMP & $04-04-91$ & USGS & $N$ & 20.72 & $S$ & $S$ \\
\hline & & $10-22-91$ & USGS & $\mathrm{N}$ & 24.52 & $\mathrm{~S}$ & $S$ \\
\hline \multirow[t]{2}{*}{$49 U 76$} & 227CPCK & $04-03-91$ & USGS & $\mathrm{U}$ & 11.77 & $S$ & $\mathbf{S}$ \\
\hline & & $10-23-91$ & USGS & $\mathrm{U}$ & 16.22 & $S$ & $S$ \\
\hline \multirow[t]{2}{*}{$49 \mathrm{U} 77$} & $227 \mathrm{CPCK}$ & $04-03-91$ & USGS & $\mathrm{U}$ & -- & $\mathrm{F}$ & $\mathrm{X}$ \\
\hline & & $10-23-91$ & USGS & $\mathrm{U}$ & 2.35 & $S$ & $\mathbf{S}$ \\
\hline \multirow[t]{2}{*}{$49 U 78$} & $227 \mathrm{CPCK}$ & $04-03-91$ & USGS & $\mathrm{U}$ & 9.23 & $\mathrm{~S}$ & $S$ \\
\hline & & $10-23-91$ & USGS & $\mathrm{U}$ & 12.69 & $S$ & $S$ \\
\hline \multirow[t]{2}{*}{$49 \mathrm{U} 79$} & $227 \mathrm{CPCK}$ & $04-03-91$ & USGS & $\mathrm{U}$ & 3.05 & $S$ & $\mathrm{~S}$ \\
\hline & & $10-23-91$ & USGS & $\mathrm{U}$ & 5.71 & $S$ & $\mathrm{~S}$ \\
\hline \multirow[t]{2}{*}{$49 \mathrm{~V} 1$} & 227TRKR & $04-26-91$ & USGS & W & 9.13 & $S$ & $S$ \\
\hline & & $10-29-91$ & USGS & W & 11.95 & $S$ & $\mathrm{~S}$ \\
\hline \multirow[t]{2}{*}{$49 \mathrm{~V} 43$} & 227MDLD & $03-27-91$ & USGS & $\mathrm{N}$ & 7.94 & $\mathrm{R}$ & $\mathrm{S}$ \\
\hline & & $10-23-91$ & USGS & $\mathrm{N}$ & 13.86 & $\mathrm{R}$ & $\mathrm{S}$ \\
\hline \multirow[t]{2}{*}{$49 V 49$} & 377CHLH & $03-22-91$ & USGS & 1.8 & 33.56 & $\mathrm{R}$ & $\mathrm{S}$ \\
\hline & & $10-22-91$ & USGS & 2.6 & 79.06 & $\mathrm{R}$ & $S$ \\
\hline \multirow[t]{2}{*}{$49 \mathrm{~V} 50$} & $377 \mathrm{CHLH}$ & $03-22-91$ & USGS & 1.6 & 33.98 & $\mathrm{R}$ & $S$ \\
\hline & & $10-18-91$ & USGS & 2.4 & 60.42 & $\mathrm{R}$ & $\mathrm{S}$ \\
\hline \multirow[t]{2}{*}{$49 V 52$} & 377CHLH & $03-21-91$ & USGS & $\mathrm{U}$ & 41.83 & $\mathrm{~S}$ & $\mathrm{~S}$ \\
\hline & & $10-18-91$ & USGS & $\mathrm{U}$ & 49.02 & $S$ & $S$ \\
\hline \multirow[t]{2}{*}{$49 V 53$} & 377CHLH & $03-21-91$ & USGS & 3 & -- & F & $X$ \\
\hline & & $10-18-91$ & USGS & 3 & -40.43 & F & $G$ \\
\hline \multirow[t]{2}{*}{$49 V 54$} & 377CHLH & $03-21-91$ & USGS & $\mathrm{U}$ & -- & $\mathrm{F}$ & $\mathrm{X}$ \\
\hline & & $10-18-91$ & USGS & $\mathrm{U}$ & -- & F & $X$ \\
\hline \multirow[t]{2}{*}{$49 \vee 55$} & 377CHLH & $03-22-91$ & USGS & 1.5 & 61.58 & $\mathrm{R}$ & $\mathrm{S}$ \\
\hline & & $10-18-91$ & USGS & 2.1 & 78.77 & $\mathbf{R}$ & $S$ \\
\hline \multirow[t]{2}{*}{$49 V 56$} & $377 \mathrm{CHLH}$ & $03-22-91$ & USGS & 2.4 & 141.39 & $\mathrm{R}$ & S \\
\hline & & $10-22-91$ & USGS & $\mathrm{N}$ & 136.44 & $\mathrm{R}$ & $S$ \\
\hline $49 V 57$ & 377CHLH & $03-22-91$ & USGS & .6 & 204.80 & $\mathrm{R}$ & S \\
\hline & & $10-22-91$ & USGS & 1.1 & 243.86 & $\mathrm{R}$ & $S$ \\
\hline 49V 58 & 377CHLH & $03-21-91$ & USGS & 13.1 & 39.14 & $\mathrm{~S}$ & $S$ \\
\hline & & $10-18-91$ & USGS & 2.3 & 49.29 & $\mathrm{R}$ & $S$ \\
\hline $49 V 87$ & $227 \mathrm{HKGV}$ & $03-27-91$ & USGS & $\mathrm{N}$ & 14.54 & $R$ & $S$ \\
\hline & & $10-23-91$ & USGS & $\mathrm{N}$ & 26.10 & $\mathrm{R}$ & $S$ \\
\hline
\end{tabular}


Table 2. Synoptic water-level measurements for selected wells in Prince William County-Continued

[Depths are measured from land surface datum. For location of wells, refer to plate 1]

\begin{tabular}{|c|c|c|c|c|c|c|c|}
\hline $\begin{array}{c}\text { Local } \\
\text { well } \\
\text { number }\end{array}$ & $\begin{array}{c}\text { Geologic } \\
\text { unit }\end{array}$ & Date & Agency & $\begin{array}{c}\text { Recovery } \\
\text { period } \\
\text { (hours) }\end{array}$ & $\begin{array}{l}\text { Water } \\
\text { level } \\
\text { (feet) }\end{array}$ & $\begin{array}{c}\text { Site } \\
\text { status }\end{array}$ & $\begin{array}{l}\text { Method of } \\
\text { measurment }\end{array}$ \\
\hline $49 \mathrm{~V} 88$ & 377CHLH & $10-18-91$ & USGS & $\mathrm{U}$ & 113.28 & $\mathrm{~S}$ & $S$ \\
\hline $49 \vee 89$ & $377 \mathrm{CHLH}$ & $10-22-91$ & USGS & 1.3 & 118.88 & $S$ & $\mathrm{~S}$ \\
\hline \multirow[t]{2}{*}{$50 \mathrm{~T} 45 \mathrm{C}$} & 231BLBF & $04-02-91$ & USGS & $\mathrm{W}$ & 18.86 & $\mathbf{S}$ & S \\
\hline & & $10-25-91$ & USGS & W & 22.19 & $\mathbf{S}$ & $\mathbf{S}$ \\
\hline \multirow[t]{2}{*}{$50 \mathrm{~T} 46 \mathrm{~F}$} & 231PLVL & $04-02-91$ & USGS & W & 33.61 & $\mathbf{S}$ & $\mathrm{S}$ \\
\hline & & $10-25-91$ & USGS & W & 36.30 & $\mathrm{~S}$ & $\mathrm{~S}$ \\
\hline \multirow[t]{2}{*}{$50 \mathrm{~T} 47 \mathrm{~F}$} & 231BLBF & $04-02-91$ & USGS & W & 11.08 & $S$ & $\mathbf{S}$ \\
\hline & & $10-25-91$ & USGS & W & 14.71 & $\mathrm{~S}$ & $\mathrm{~S}$ \\
\hline \multirow[t]{2}{*}{$50 \mathrm{~T} 48 \mathrm{~F}$} & 231BLBF & $04-02-91$ & USGS & W & 34.65 & $\mathbf{S}$ & $\mathbf{S}$ \\
\hline & & $10-25-91$ & USGS & W & 38.24 & $S$ & $\mathbf{S}$ \\
\hline \multirow[t]{2}{*}{$50 \mathrm{~T} 54$} & 231BLBF & $03-02-91$ & IBM & $\mathrm{W}$ & 37.74 & $\mathbf{S}$ & $\mathrm{T}$ \\
\hline & & $10-01-91$ & IBM & W & 48.99 & $S$ & $\mathrm{~T}$ \\
\hline \multirow[t]{2}{*}{$50 \mathrm{~T} 55$} & 231BLBF & $03-01-91$ & IBM & W & 44.54 & $\mathbf{S}$ & $\mathrm{T}$ \\
\hline & & $10-02-91$ & IBM & W & 56.49 & $\mathbf{S}$ & $\mathrm{T}$ \\
\hline \multirow[t]{2}{*}{ 50T 56} & 231BLBF & $03-02-91$ & IBM & W & 34.41 & $S$ & $\mathrm{~T}$ \\
\hline & & $10-01-91$ & IBM & W & 45.46 & $\mathrm{~S}$ & $\mathrm{~T}$ \\
\hline \multirow[t]{2}{*}{$50 \mathrm{~T} 57$} & 231BLBF & $03-02-91$ & IBM & w & 28.19 & $\mathrm{~S}$ & $\mathrm{~T}$ \\
\hline & & $10-01-91$ & IBM & W & 31.79 & $S$ & $\mathrm{~T}$ \\
\hline \multirow[t]{2}{*}{$50 \mathrm{~T} 58$} & 231BLBF & $03-01-91$ & IBM & w & 24.56 & $\mathrm{~s}$ & $\mathrm{~T}$ \\
\hline & & $10-02-91$ & IBM & W & 32.06 & $\mathbf{S}$ & $\mathrm{T}$ \\
\hline \multirow[t]{2}{*}{$50 \mathrm{~T} 59$} & 231BLBF & $03-02-91$ & IBM & W & 25.56 & $\mathbf{S}$ & $\mathrm{T}$ \\
\hline & & $10-01-91$ & IBM & W & 36.21 & $\mathrm{~S}$ & $\mathrm{~T}$ \\
\hline \multirow[t]{2}{*}{$50 \mathrm{~T} 60$} & 231BLBF & $03-01-91$ & IBM & W & 53.80 & $\mathbf{S}$ & $\mathrm{T}$ \\
\hline & & $10-02-91$ & IBM & W & 62.72 & $S$ & $\mathrm{~T}$ \\
\hline \multirow[t]{2}{*}{$50 \mathrm{~T} 61$} & 231BLBF & $03-02-91$ & IBM & w & 55.75 & $\mathrm{~S}$ & $\mathrm{~T}$ \\
\hline & & $10-01-91$ & IBM & W & 64.12 & $\mathrm{~S}$ & $\mathrm{~T}$ \\
\hline \multirow[t]{2}{*}{$50 \mathrm{~T} 62$} & 231BLBF & $03-02-91$ & IBM & W & 30.96 & $S$ & $\mathrm{~T}$ \\
\hline & & $10-01-91$ & IBM & W & 38.26 & S & $\mathrm{T}$ \\
\hline \multirow[t]{2}{*}{ 50T 79} & 231BLBF & $04-05-91$ & USGS & $\mathbf{N}$ & 37.62 & $\mathrm{~S}$ & $\mathbf{S}$ \\
\hline & & $10-23-91$ & USGS & 15 & 43.91 & $\mathrm{~S}$ & $\mathrm{~S}$ \\
\hline \multirow[t]{2}{*}{$50 \mathrm{~T} 80$} & 231BLBF & $03-21-91$ & USGS & $\mathbf{N}$ & 10.71 & $S$ & $S$ \\
\hline & & $10-18-91$ & USGS & $\mathbf{N}$ & 13.88 & $\mathrm{~S}$ & $\mathrm{~S}$ \\
\hline \multirow[t]{2}{*}{$50 \mathrm{~T} 81$} & 231BLBF & $03-26-91$ & USGS & $\mathbf{N}$ & 42.09 & $\mathrm{R}$ & $\mathbf{S}$ \\
\hline & & $10-23-91$ & USGS & $\mathrm{N}$ & 49.01 & $\mathrm{~S}$ & $\mathbf{S}$ \\
\hline \multirow[t]{2}{*}{$50 \mathrm{~T} 82$} & 231PLVL & $03-28-91$ & USGS & $\mathrm{U}$ & 52.01 & $S$ & $S$ \\
\hline & & $10-23-91$ & USGS & $\mathrm{U}$ & 85.69 & $\mathrm{~S}$ & $\mathrm{~S}$ \\
\hline \multirow[t]{2}{*}{$50 \mathrm{~T} 83$} & 231PLVL & $03-28-91$ & USGS & $\mathbf{N}$ & 101.96 & $\mathrm{R}$ & $\mathrm{S}$ \\
\hline & & $10-22-91$ & USGS & $\mathbf{N}$ & 105.82 & $\mathrm{R}$ & $S$ \\
\hline $50 U 1 F$ & 231BLBF & $10-24-91$ & USGS & $\mathbf{N}$ & 59.01 & $S$ & $\mathrm{~S}$ \\
\hline \multirow[t]{2}{*}{$50 \mathrm{U} 97$} & 227DIBS & $03-27-91$ & USGS & $\mathbf{N}$ & 162.37 & $\mathrm{R}$ & $S$ \\
\hline & & $10-24-91$ & USGS & $\mathrm{N}$ & 170.64 & $\mathrm{R}$ & $\mathrm{S}$ \\
\hline \multirow[t]{2}{*}{$50 \mathrm{U} 110$} & 227CPCK & $03-28-91$ & USGS & $\mathrm{N}$ & 27.81 & $\mathrm{R}$ & $\mathrm{S}$ \\
\hline & & $10-23-91$ & USGS & $\mathrm{N}$ & 19.69 & $\mathrm{R}$ & $S$ \\
\hline \multirow[t]{2}{*}{$50 \mathrm{U} 119$} & 231BLBF & 03-01-91 & IBM & W & 16.12 & $\mathrm{~S}$ & $\mathrm{~T}$ \\
\hline & & $10-02-91$ & IBM & W & 18.56 & $\mathrm{~S}$ & $\mathrm{~T}$ \\
\hline $50 \mathrm{U} 120$ & 231BLBF & $03-02-91$ & IBM & W & 16.44 & $S$ & $\mathrm{~T}$ \\
\hline & & $10-01-91$ & IBM & W & 19.04 & $S$ & $\mathrm{~T}$ \\
\hline
\end{tabular}


Table 2. Synoptic water-level measurements for selected wells in Prince William County-Continued

[Depths are measured from land surface datum; negative numbers indicate values above land surface; dashes (--) indicate that water levels were not measured. For location of wells, refer to plate 1]

\begin{tabular}{|c|c|c|c|c|c|c|c|}
\hline $\begin{array}{c}\text { Local } \\
\text { well } \\
\text { number }\end{array}$ & $\begin{array}{l}\text { Geologic } \\
\text { unit }\end{array}$ & Date & Agency & $\begin{array}{c}\text { Recovery } \\
\text { period } \\
\text { (hours) }\end{array}$ & $\begin{array}{l}\text { Water } \\
\text { level } \\
\text { (feet) }\end{array}$ & $\begin{array}{c}\text { Site } \\
\text { status }\end{array}$ & $\begin{array}{l}\text { Method of } \\
\text { measurmen }\end{array}$ \\
\hline \multirow{2}{*}{$50 \mathrm{U} 122$} & 231BLBF & $03-20-91$ & USGS & 1.9 & 69.88 & $\mathrm{R}$ & $S$ \\
\hline & & $10-16-91$ & USGS & 1.4 & 72.41 & $\mathrm{R}$ & $S$ \\
\hline \multirow[t]{2}{*}{$50 \mathrm{U} 123$} & 231BLBF & $03-20-91$ & USGS & 2.8 & 87.98 & $\mathrm{R}$ & $S$ \\
\hline & & $10-17-91$ & USGS & 2.1 & 111.23 & $\mathbf{R}$ & $S$ \\
\hline \multirow[t]{2}{*}{$50 \mathrm{U} 124$} & 231BLBF & $03-21-91$ & USGS & $\mathbf{N}$ & 23.97 & $\mathrm{R}$ & $S$ \\
\hline & & $10-17-91$ & USGS & $\mathrm{U}$ & 22.87 & $S$ & $S$ \\
\hline \multirow[t]{2}{*}{$50 \mathrm{U} 125$} & $227 \mathrm{CPCK}$ & $03-21-91$ & USGS & $\mathbf{N}$ & 49.81 & $\mathrm{R}$ & $S$ \\
\hline & & $10-17-91$ & USGS & $\mathrm{U}$ & 45.44 & $\mathrm{~S}$ & $S$ \\
\hline \multirow[t]{2}{*}{$50 \mathrm{U} 126$} & 227TMMP & $03-21-91$ & USGS & 0.4 & 23.95 & $\mathrm{R}$ & $S$ \\
\hline & & $10-18-91$ & USGS & .8 & 40.51 & $\mathrm{R}$ & $S$ \\
\hline \multirow[t]{2}{*}{$50 \mathrm{U} 127$} & 227TMMP & 04-04-91 & USGS & $\mathrm{U}$ & 1.16 & $S$ & $S$ \\
\hline & & $10-24-91$ & USGS & $\mathrm{U}$ & 4.38 & $\mathrm{~S}$ & $\mathbf{S}$ \\
\hline \multirow[t]{2}{*}{$50 \mathrm{U} 128$} & 227DIBS & $03-28-91$ & USGS & $\mathrm{N}$ & 35.79 & $S$ & $S$ \\
\hline & & $10-17-91$ & USGS & 1.2 & 46.59 & $\mathrm{R}$ & $S$ \\
\hline \multirow{2}{*}{$50 \mathrm{U} 132$} & 227TMMP & $04-04-91$ & USGS & $\mathrm{U}$ & -- & $\mathrm{F}$ & $\mathrm{X}$ \\
\hline & & $10-24-91$ & USGS & U & -0.58 & $\mathrm{~S}$ & $S$ \\
\hline \multirow[t]{2}{*}{$50 U 133$} & $227 \mathrm{CPCK}$ & $04-04-91$ & USGS & $\mathbf{N}$ & 44.25 & $\mathrm{R}$ & $S$ \\
\hline & & $10-21-91$ & USGS & $\mathrm{N}$ & 73.51 & $\mathrm{R}$ & $S$ \\
\hline \multirow[t]{2}{*}{$50 U 135$} & 231BLBF & $03-21-91$ & USGS & $\mathrm{N}$ & 13.83 & $S$ & $S$ \\
\hline & & $10-18-91$ & USGS & $\mathbf{N}$ & 17.83 & $S$ & $S$ \\
\hline \multirow[t]{2}{*}{$50 \mathrm{U} 136$} & 227GCRK & $04-01-91$ & USGS & $\mathbf{N}$ & 18.80 & $S$ & S \\
\hline & & $10-24-91$ & USGS & $\mathrm{N}$ & 33.51 & $S$ & $S$ \\
\hline \multirow[t]{2}{*}{$50 \mathrm{U} 137$} & 227DIBS & $03-28-91$ & USGS & $\mathrm{N}$ & 5.29 & $S$ & $S$ \\
\hline & & $10-17-91$ & USGS & .02 & 8.41 & $S$ & $S$ \\
\hline \multirow[t]{2}{*}{$50 \mathrm{~V} 89$} & $227 \mathrm{CPCK}$ & $03-27-91$ & USGS & $\mathrm{U}$ & 26.07 & $S$ & $S$ \\
\hline & & $10-23-91$ & USGS & $N$ & 38.84 & $S$ & $S$ \\
\hline \multirow[t]{2}{*}{$51 \mathrm{~S} 5$} & 360LGRV & $05-14-91$ & USGS & $\mathrm{N}$ & 30.43 & $S$ & $S$ \\
\hline & & $10-16-91$ & USGS & $\mathrm{N}$ & 35.84 & $S$ & $S$ \\
\hline \multirow[t]{2}{*}{$51 \mathrm{~S} 7$} & 360LGRV & $03-15-91$ & USGS & W & 3.82 & S & $S$ \\
\hline & & $10-11-91$ & USGS & W & 12.12 & $S$ & $S$ \\
\hline \multirow[t]{2}{*}{$51 \mathrm{~T} 1 \mathrm{~A}$} & 231PLVL & $03-26-91$ & USGS & $\mathrm{N}$ & 45.57 & $S$ & $S$ \\
\hline & & $10-23-91$ & USGS & $\mathrm{N}$ & 49.65 & $S$ & $\mathrm{~S}$ \\
\hline \multirow[t]{2}{*}{$51 \mathrm{~T} 3$} & $360 \mathrm{LKJK}$ & $03-19-91$ & USGS & $\mathrm{U}$ & 37.77 & $S$ & $S$ \\
\hline & & $10-15-91$ & USGS & $U$ & 41.05 & $\mathbf{S}$ & $\mathrm{S}$ \\
\hline \multirow[t]{2}{*}{$51 \mathrm{~T} 29 \mathrm{E}$} & 360PLLT & $03-18-91$ & USGS & .3 & 94.33 & $\mathbf{R}$ & $\mathrm{S}$ \\
\hline & & $10-15-91$ & USGS & 1.4 & 98.23 & $\mathbf{R}$ & $S$ \\
\hline \multirow[t]{2}{*}{$51 \mathrm{~T} 108$} & 231PLVL & 03-02-91 & IBM & W & 16.03 & $S$ & $\mathrm{~T}$ \\
\hline & & $10-01-91$ & IBM & W & 19.00 & $S$ & $T$ \\
\hline \multirow[t]{2}{*}{$51 \mathrm{~T} 117$} & 350MTTL & 03-18-91 & USGS & 1 & 135.02 & $\mathbf{R}$ & $\mathbf{S}$ \\
\hline & & $10-15-91$ & USGS & 3.1 & 101.76 & $\mathbf{R}$ & $S$ \\
\hline \multirow{2}{*}{$51 T 118$} & 350MTTL & $03-18-91$ & USGS & 1 & 79.38 & $\mathbf{R}$ & $\mathbf{S}$ \\
\hline & & $10-15-91$ & USGS & 1.7 & 48.72 & $\mathbf{R}$ & $S$ \\
\hline $51 \mathrm{~T} 119$ & 350MTTL & 03-18-91 & USGS & 1 & 19.09 & $\mathbf{R}$ & $S$ \\
\hline & & $10-15-91$ & USGS & 1.9 & 26.41 & $\mathbf{R}$ & $S$ \\
\hline $51 \mathrm{~T} 120$ & 350MTTL & $03-18-91$ & USGS & .6 & 60.43 & $\mathbf{R}$ & $\mathbf{S}$ \\
\hline & & $10-15-91$ & USGS & 1.7 & 48.62 & $\mathbf{R}$ & $\mathbf{S}$ \\
\hline 51T121 & 350MTTL & $03-19-91$ & USGS & $\mathbf{U}$ & 4.90 & $S$ & $S$ \\
\hline & & $10-15-91$ & USGS & $\mathrm{U}$ & 13.61 & $S$ & $S$ \\
\hline
\end{tabular}


Table 2. Synoptic water-level measurements for selected wells in Prince William County-Continued

[Depths are measured from land surface datum. For location of wells, refer to plate 1]

\begin{tabular}{|c|c|c|c|c|c|c|c|}
\hline $\begin{array}{c}\text { Local } \\
\text { well } \\
\text { number }\end{array}$ & $\begin{array}{c}\text { Geologic } \\
\text { unit }\end{array}$ & Date & Agency & $\begin{array}{c}\text { Recovery } \\
\text { period } \\
\text { (hours) }\end{array}$ & $\begin{array}{l}\text { Water } \\
\text { level } \\
\text { (feet) }\end{array}$ & $\begin{array}{c}\text { Site } \\
\text { status }\end{array}$ & $\begin{array}{l}\text { Method of } \\
\text { measurment }\end{array}$ \\
\hline \multirow[t]{2}{*}{$51 \mathrm{~T} 122$} & $360 \mathrm{LKJK}$ & $03-19-91$ & USGS & 1.5 & 44.93 & $\mathbf{R}$ & $S$ \\
\hline & & $10-16-91$ & USGS & 1.6 & 49.53 & $\mathrm{R}$ & $S$ \\
\hline $51 \mathrm{~T} 123$ & $360 \mathrm{PCBC}$ & $10-15-91$ & USGS & $\mathrm{U}$ & 53.85 & $S$ & $S$ \\
\hline \multirow[t]{2}{*}{$51 \mathrm{~T} 140$} & $360 \mathrm{LKJK}$ & $03-19-91$ & USGS & $\mathbf{U}$ & 24.15 & $\mathrm{~S}$ & $S$ \\
\hline & & $10-18-91$ & USGS & $\mathrm{U}$ & 29.84 & $\mathrm{~S}$ & $S$ \\
\hline \multirow[t]{2}{*}{$51 \mathrm{~T} 166$} & 360PLLT & $03-19-91$ & USGS & 2 & 67.68 & $\mathbf{R}$ & $S$ \\
\hline & & $10-15-91$ & USGS & 1.6 & 78.91 & $\mathrm{R}$ & $S$ \\
\hline \multirow[t]{2}{*}{$51 \mathrm{~T} 167$} & $360 \mathrm{LKJK}$ & $04-04-91$ & USGS & 2.8 & 39.40 & $\mathrm{~S}$ & $S$ \\
\hline & & $10-23-91$ & USGS & 6.3 & 44.83 & $S$ & $S$ \\
\hline \multirow[t]{2}{*}{$51 \mathrm{~T} 171$} & $360 \mathrm{LKJK}$ & $03-19-91$ & USGS & 0.8 & 55.52 & $\mathrm{R}$ & $S$ \\
\hline & & $10-16-91$ & USGS & 2.2 & 52.99 & $\mathrm{R}$ & $S$ \\
\hline \multirow[t]{2}{*}{$51 \mathrm{~T} 172$} & $360 \mathrm{PCBC}$ & $03-18-91$ & USGS & $\mathrm{U}$ & 37.07 & $\mathrm{~S}$ & $S$ \\
\hline & & $10-15-91$ & USGS & 1.5 & 44.32 & $\mathrm{R}$ & $S$ \\
\hline \multirow[t]{2}{*}{$51 \mathrm{~T} 173$} & $360 \mathrm{PCBC}$ & $03-18-91$ & USGS & .9 & 42.57 & $\mathbf{R}$ & $S$ \\
\hline & & $10-15-91$ & USGS & 4.8 & 83.60 & $\mathrm{R}$ & $S$ \\
\hline \multirow{2}{*}{$51 \mathrm{~T} 174$} & $360 \mathrm{LKJK}$ & $03-19-91$ & USGS & 2.9 & 63.02 & $\mathbf{R}$ & $S$ \\
\hline & & $10-16-91$ & USGS & 1.2 & 66.43 & $\mathrm{R}$ & $S$ \\
\hline \multirow[t]{2}{*}{$51 T 175$} & 360PLLT & $03-19-91$ & USGS & 3.2 & 54.21 & $\mathrm{R}$ & $S$ \\
\hline & & $10-16-91$ & USGS & .8 & 61.20 & $\mathrm{R}$ & $S$ \\
\hline \multirow[t]{2}{*}{$51 \mathrm{~T} 176$} & $360 \mathrm{LKJK}$ & $03-19-91$ & USGS & $\mathrm{U}$ & 26.68 & $\mathrm{~S}$ & $S$ \\
\hline & & $10-16-91$ & USGS & $\mathbf{U}$ & 29.81 & $\mathrm{~S}$ & $S$ \\
\hline \multirow[t]{2}{*}{$51 \mathrm{U} 4 \mathrm{H}$} & 231PLVL & $03-26-91$ & USGS & $\mathrm{N}$ & 1.72 & $\mathrm{~S}$ & $S$ \\
\hline & & $10-23-91$ & USGS & $\mathbf{N}$ & 5.25 & $\mathrm{~S}$ & $S$ \\
\hline \multirow[t]{2}{*}{$51 \mathrm{U} 7 \mathrm{H}$} & 231PLVL & $03-25-91$ & USGS & $\mathbf{U}$ & 63.03 & $\mathrm{~S}$ & $S$ \\
\hline & & $10-23-91$ & USGS & $\mathbf{U}$ & 65.79 & $\mathrm{~S}$ & $S$ \\
\hline \multirow[t]{2}{*}{$51 \mathrm{U} 9 \mathrm{G}$} & 231PLVL & $03-26-91$ & USGS & $\mathbf{U}$ & 88.89 & $\mathrm{~S}$ & $S$ \\
\hline & & $10-16-91$ & USGS & $\mathrm{U}$ & 95.45 & $\mathrm{~S}$ & $\mathrm{~S}$ \\
\hline \multirow[t]{2}{*}{$51 \mathrm{U} 10$} & 231PLVL & $03-19-91$ & USGS & 2.1 & 79.32 & $\mathrm{R}$ & $S$ \\
\hline & & $10-16-91$ & USGS & 1.2 & 89.18 & $\mathrm{R}$ & $S$ \\
\hline \multirow[t]{2}{*}{$51 \mathrm{U} 11$} & 231PLVL & $03-19-91$ & USGS & 2.4 & 113.58 & $\mathrm{R}$ & $S$ \\
\hline & & $10-16-91$ & USGS & .5 & 140.80 & $\mathrm{R}$ & $S$ \\
\hline \multirow[t]{2}{*}{$51 \mathrm{U} 16$} & 231PLVL & $03-19-91$ & USGS & 1 & 131.30 & $\mathrm{R}$ & $S$ \\
\hline & & $10-16-91$ & USGS & 2 & 144.78 & $\mathrm{R}$ & $S$ \\
\hline \multirow[t]{2}{*}{$51 \mathrm{U} 19 \mathrm{D}$} & 231BLBF & $03-20-91$ & USGS & 1.8 & 46.80 & $\mathrm{R}$ & $\mathrm{S}$ \\
\hline & & $10-17-91$ & USGS & 1.8 & 56.94 & $\mathrm{R}$ & $S$ \\
\hline \multirow[t]{2}{*}{$51 \mathrm{U} 20 \mathrm{G}$} & 231PLVL & $03-26-91$ & USGS & $\mathrm{U}$ & 75.87 & $S$ & $S$ \\
\hline & & $10-16-91$ & USGS & $\mathbf{U}$ & 83.76 & $S$ & $S$ \\
\hline \multirow[t]{2}{*}{$51 \mathrm{U} 92 \mathrm{G}$} & 231PLVL & $03-26-91$ & USGS & U & 60.98 & $S$ & $S$ \\
\hline & & $10-24-91$ & USGS & $\mathbf{U}$ & 63.24 & $\mathrm{~S}$ & $S$ \\
\hline \multirow[t]{2}{*}{$51 \mathrm{U} 94 \mathrm{G}$} & 231PLVL & $03-26-91$ & USGS & $\mathbf{U}$ & 77.04 & $\mathbf{S}$ & $S$ \\
\hline & & $10-24-91$ & USGS & $\mathrm{U}$ & 84.17 & $S$ & $S$ \\
\hline \multirow[t]{2}{*}{$51 \mathrm{U} 99 \mathrm{G}$} & 231BLBF & $03-20-91$ & USGS & 1.4 & 78.89 & $\mathrm{R}$ & $S$ \\
\hline & & $10-17-91$ & USGS & 1.1 & 81.80 & $\mathrm{R}$ & $S$ \\
\hline 51U100G & 231BLBF & $03-20-91$ & USGS & 2.5 & 42.70 & $\mathrm{R}$ & $S$ \\
\hline & & $10-17-91$ & USGS & 1.6 & 52.66 & $\mathrm{R}$ & $S$ \\
\hline $51 \mathrm{U} 101 \mathrm{G}$ & 231BLBF & $03-20-91$ & USGS & 6.4 & 112.89 & $\mathrm{R}$ & $\mathrm{T}$ \\
\hline & & $10-17-91$ & USGS & 4 & 135.53 & $\mathrm{R}$ & $\mathrm{T}$ \\
\hline
\end{tabular}


Table 2. Synoptic water-level measurements for selected wells in Prince William County-Continued

[Depths are measured from land surface datum. For location of wells, refer to plate 1]

\begin{tabular}{|c|c|c|c|c|c|c|c|}
\hline $\begin{array}{l}\text { Local } \\
\text { well } \\
\text { number }\end{array}$ & $\begin{array}{c}\text { Geologic } \\
\text { unit }\end{array}$ & Date & Agency & $\begin{array}{l}\text { Recovery } \\
\text { period } \\
\text { (hours) }\end{array}$ & $\begin{array}{l}\text { Water } \\
\text { level } \\
\text { (feet) }\end{array}$ & $\begin{array}{l}\text { Site } \\
\text { status }\end{array}$ & $\begin{array}{l}\text { Method of } \\
\text { measurment }\end{array}$ \\
\hline \multirow[t]{2}{*}{ 51U102D } & 231BLBF & $03-20-91$ & USGS & 2.1 & 35.25 & $\mathbf{R}$ & $S$ \\
\hline & & $10-17-91$ & USGS & 2.2 & 46.86 & $\mathbf{R}$ & $S$ \\
\hline \multirow[t]{2}{*}{$51 \mathrm{U} 103 \mathrm{G}$} & 231BLBF & $03-25-91$ & USGS & 0.7 & 93.50 & $\mathrm{R}$ & C \\
\hline & & $10-23-91$ & USGS & 1.2 & 74.99 & $\mathbf{R}$ & $S$ \\
\hline \multirow[t]{2}{*}{ 51U104G } & 231PLVL & $04-12-91$ & USGS & .9 & 64.60 & $\mathrm{R}$ & $S$ \\
\hline & & $10-23-91$ & USGS & $\mathrm{U}$ & 65.78 & $\mathrm{~S}$ & $S$ \\
\hline \multirow[t]{2}{*}{ 51U105G } & $231 \mathrm{BLBF}$ & $03-20-91$ & USGS & 5.9 & 99.57 & $\mathbf{R}$ & $S$ \\
\hline & & $10-17-91$ & USGS & 6.8 & 103.95 & $R$ & $S$ \\
\hline \multirow[t]{2}{*}{ 51U106D } & 231BLBF & $03-20-91$ & USGS & 2.8 & 41.14 & $R$ & $\mathrm{~S}$ \\
\hline & & $10-17-91$ & USGS & 2.1 & 50.12 & $\mathbf{R}$ & $S$ \\
\hline \multirow[t]{2}{*}{$51 \mathrm{U} 111$} & 231BLBF & $03-26-91$ & USGS & $\mathrm{U}$ & 38.79 & S & $\mathbf{S}$ \\
\hline & & $10-24-91$ & USGS & $\mathrm{U}$ & 45.18 & $S$ & $S$ \\
\hline \multirow[t]{2}{*}{$51 \mathrm{U} 112$} & 231BLBF & $03-02-91$ & IBM & W & 53.44 & $S$ & $\mathrm{~T}$ \\
\hline & & $10-01-91$ & IBM & W & 63.64 & $S$ & $\mathrm{~T}$ \\
\hline \multirow[t]{2}{*}{$51 \mathrm{U} 113$} & 231BLBF & $03-02-91$ & IBM & W & 47.53 & $S$ & $\mathrm{~T}$ \\
\hline & & $10-01-91$ & IBM & W & 56.08 & $S$ & $\mathrm{~T}$ \\
\hline \multirow[t]{2}{*}{$51 \mathrm{U} 114$} & 231BLBF & $03-02-91$ & IBM & W & 71.03 & $S$ & $\mathrm{~T}$ \\
\hline & & $10-01-91$ & IBM & W & 79.33 & $S$ & $\mathrm{~T}$ \\
\hline \multirow[t]{2}{*}{ 51U115 } & 231BLBF & $03-02-91$ & IBM & W & 46.94 & $S$ & $\mathrm{~T}$ \\
\hline & & $10-01-91$ & IBM & W & 53.99 & $\mathrm{~S}$ & $\mathrm{~T}$ \\
\hline \multirow[t]{2}{*}{ 51U116 } & 231BLBF & $03-02-91$ & IBM & W & 86.88 & $S$ & $\mathrm{~T}$ \\
\hline & & $10-01-91$ & IBM & W & 93.23 & S & $\mathrm{T}$ \\
\hline \multirow[t]{2}{*}{$51 \mathrm{U} 117$} & 231BLBF & $03-02-91$ & IBM & W & 88.34 & $S$ & $\mathrm{~T}$ \\
\hline & & $10-01-91$ & IBM & W & 95.74 & $S$ & $\mathrm{~T}$ \\
\hline \multirow[t]{2}{*}{ 51U118 } & 231BLBF & 03-02-91 & IBM & W & 76.87 & $S$ & $\mathrm{~T}$ \\
\hline & & $10-01-91$ & IBM & W & 88.24 & $S$ & $\mathrm{~T}$ \\
\hline \multirow[t]{2}{*}{$51 \mathrm{U} 120$} & 231BLBF & $03-01-91$ & IBM & W & 59.96 & $S$ & $\mathrm{~T}$ \\
\hline & & $10-02-91$ & IBM & W & 70.56 & $S$ & $\mathrm{~T}$ \\
\hline \multirow[t]{2}{*}{$51 \mathrm{U} 121$} & 231BLBF & $03-02-91$ & IBM & W & 61.48 & $S$ & $\mathrm{~T}$ \\
\hline & & $10-01-91$ & IBM & W & 71.43 & $S$ & $\mathrm{~T}$ \\
\hline \multirow[t]{2}{*}{$51 \mathrm{U} 122$} & 231BLBF & $03-01-91$ & IBM & W & 57.70 & $S$ & $\mathrm{~T}$ \\
\hline & & $10-02-91$ & IBM & W & 65.80 & $S$ & $\mathrm{~T}$ \\
\hline \multirow[t]{2}{*}{$51 \mathrm{U} 123$} & 231BLBF & $03-02-91$ & IBM & W & 57.42 & $S$ & $\mathrm{~T}$ \\
\hline & & $10-01-91$ & IBM & W & 65.62 & $S$ & $\mathrm{~T}$ \\
\hline \multirow[t]{2}{*}{$51 \mathrm{U} 124$} & 231BLBF & $03-01-91$ & IBM & W & 44.42 & $S$ & $\mathrm{~T}$ \\
\hline & & $10-02-91$ & IBM & W & 53.87 & $S$ & $\mathrm{~T}$ \\
\hline \multirow[t]{2}{*}{$51 \mathrm{U} 125$} & $231 B L B F$ & 03-02-91 & IBM & W & 54.55 & $S$ & $\mathrm{~T}$ \\
\hline & & $10-01-91$ & IBM & W & 62.20 & $S$ & $\mathrm{~T}$ \\
\hline \multirow[t]{2}{*}{$51 \mathrm{U} 126$} & 231BLBF & $03-01-91$ & IBM & W & 67.59 & $S$ & $\mathrm{~T}$ \\
\hline & & $10-02-91$ & IBM & W & 77.99 & $S$ & $\mathrm{~T}$ \\
\hline \multirow[t]{2}{*}{$51 \mathrm{U} 127$} & 231BLBF & $03-02-91$ & IBM & W & 69.93 & $S$ & $\mathrm{~T}$ \\
\hline & & $10-01-91$ & IBM & W & 79.88 & $S$ & $\mathrm{~T}$ \\
\hline $51 \mathrm{U} 129$ & 231BLBF & $03-02-91$ & IBM & W & 45.76 & $\mathrm{~S}$ & $T$ \\
\hline & & $10-01-91$ & IBM & W & 56.16 & $\mathrm{~S}$ & $\mathrm{~T}$ \\
\hline $51 \mathrm{U} 130$ & 231BLBF & $03-01-91$ & IBM & W & 44.23 & $\mathrm{~S}$ & $\mathrm{~T}$ \\
\hline & & $10-02-91$ & IBM & W & 51.88 & $S$ & $\mathrm{~T}$ \\
\hline $51 \mathrm{U} 131$ & 231BLBF & $03-02-91$ & IBM & W & 45.79 & $S$ & $\mathrm{~T}$ \\
\hline & & $10-01-91$ & IBM & W & 53.69 & $S$ & $\mathrm{~T}$ \\
\hline
\end{tabular}


Table 2. Synoptic water-level measurements for selected wells in Prince William County-Continued

[Depths are measured from land surface datum; negative numbers indicate values above land surface; dashes (--) indicate that water levels were not measured. For location of wells, refer to plate 1]

\begin{tabular}{|c|c|c|c|c|c|c|c|}
\hline $\begin{array}{l}\text { Local } \\
\text { well } \\
\text { number }\end{array}$ & $\begin{array}{l}\text { Geologic } \\
\text { unit }\end{array}$ & Date & Agency & $\begin{array}{c}\text { Recovery } \\
\text { period } \\
\text { (hours) }\end{array}$ & $\begin{array}{l}\text { Water } \\
\text { level } \\
\text { (feet) }\end{array}$ & $\begin{array}{c}\text { Site } \\
\text { status }\end{array}$ & $\begin{array}{l}\text { Method of } \\
\text { measurment }\end{array}$ \\
\hline \multirow[t]{2}{*}{$51 \mathrm{U} 132$} & 231PLVL & $03-01-91$ & IBM & w & 67.57 & $S$ & $\mathrm{~T}$ \\
\hline & & $10-02-91$ & IBM & W & 74.84 & $S$ & $\mathrm{~T}$ \\
\hline \multirow[t]{2}{*}{$51 U 133$} & 231PLVL & $03-02-91$ & IBM & W & 72.55 & $S$ & $\mathrm{~T}$ \\
\hline & & $10-01-91$ & IBM & W & 78.80 & $S$ & $\mathrm{~T}$ \\
\hline \multirow{2}{*}{$51 \mathrm{U} 134$} & 231PLVL & $03-02-91$ & IBM & W & 53.30 & $S$ & $\mathrm{~T}$ \\
\hline & & $10-01-91$ & IBM & W & 62.10 & $S$ & $\mathrm{~T}$ \\
\hline \multirow[t]{2}{*}{$51 \mathrm{U} 135$} & 231BLBF & $03-01-91$ & IBM & w & 73.82 & $S$ & $\mathrm{~T}$ \\
\hline & & $10-02-91$ & IBM & w & 85.72 & $S$ & $\mathrm{~T}$ \\
\hline \multirow[t]{2}{*}{$51 \mathrm{U} 136$} & 231BLBF & $03-02-91$ & IBM & w & 65.63 & $S$ & $\mathrm{~T}$ \\
\hline & & $10-01-91$ & IBM & W & 75.73 & $\mathrm{~S}$ & $\mathrm{~T}$ \\
\hline \multirow[t]{2}{*}{$51 \mathrm{U} 137$} & 231BLBF & $03-02-91$ & IBM & W & 111.56 & $S$ & $\mathrm{~T}$ \\
\hline & & $10-01-91$ & IBM & w & 113.81 & $S$ & $\mathrm{~T}$ \\
\hline \multirow[t]{2}{*}{$51 \mathrm{U} 138$} & 231PLVL & $03-02-91$ & IBM & w & 19.52 & $S$ & $\mathrm{~T}$ \\
\hline & & $10-01-91$ & IBM & W & 25.82 & $\mathrm{~S}$ & $\mathrm{~T}$ \\
\hline \multirow[t]{2}{*}{$51 \mathrm{U} 139$} & 231PLVL & 03-02-91 & IBM & W & 66.58 & $S$ & $\mathrm{~T}$ \\
\hline & & $10-01-91$ & IBM & w & 73.03 & $\mathrm{~S}$ & $\mathrm{~T}$ \\
\hline \multirow[t]{2}{*}{$51 U 140$} & 231BLBF & $03-01-91$ & IBM & W & 69.90 & $\mathrm{~S}$ & $\mathrm{~T}$ \\
\hline & & $10-02-91$ & IBM & W & 78.45 & $S$ & $\mathrm{~T}$ \\
\hline \multirow{2}{*}{$51 \mathrm{U} 141$} & 231BLBF & $03-02-91$ & IBM & W & 27.76 & $S$ & $\mathrm{~T}$ \\
\hline & & $10-01-91$ & IBM & W & 35.26 & $S$ & $\mathrm{~T}$ \\
\hline \multirow[t]{2}{*}{$51 \mathrm{U} 142$} & 231PLVL & $03-02-91$ & IBM & w & 46.92 & $S$ & $\mathrm{~T}$ \\
\hline & & $10-01-91$ & IBM & W & 53.27 & $S$ & $\mathrm{~T}$ \\
\hline \multirow[t]{2}{*}{$52 S 3$} & 377CРMC & $05-14-91$ & USGS & $\mathrm{U}$ & 2.50 & $S$ & $S$ \\
\hline & & $10-16-91$ & USGS & $\mathrm{U}$ & 5.04 & $S$ & $S$ \\
\hline \multirow[t]{2}{*}{$52 S 4$} & 217РТМC & $03-15-91$ & USGS & W & 18.62 & $S$ & $S$ \\
\hline & & $10-11-91$ & USGS & W & 19.05 & $S$ & $S$ \\
\hline \multirow[t]{2}{*}{$52 \mathrm{~S} 5$} & 217PTMC & $03-15-91$ & USGS & W & 17.56 & $S$ & $S$ \\
\hline & & $10-11-91$ & USGS & W & 17.99 & $S$ & $S$ \\
\hline \multirow[t]{2}{*}{$52 \mathrm{~S} 12$} & 361QNTC & $05-22-91$ & USGS & $\mathrm{U}$ & 37.86 & $S$ & $S$ \\
\hline & & $10-17-91$ & USGS & $\mathrm{U}$ & 39.28 & $S$ & $S$ \\
\hline \multirow[t]{2}{*}{$52 \mathrm{~S} 14$} & $377 \mathrm{CPMC}$ & $05-14-91$ & USGS & $\mathrm{N}$ & 64.36 & $S$ & $S$ \\
\hline & & $10-16-91$ & USGS & $\mathrm{N}$ & 77.67 & $\mathbf{R}$ & $S$ \\
\hline \multirow[t]{2}{*}{$52 \mathrm{~S} 18$} & 360LGRV & $05-14-91$ & USGS & 96 & 25.49 & $S$ & $S$ \\
\hline & & $10-16-91$ & USGS & 48 & 30.06 & $S$ & $S$ \\
\hline \multirow[t]{2}{*}{$52 S 20$} & 377CPMC & $05-14-91$ & USGS & $\mathrm{U}$ & 15.20 & $S$ & $S$ \\
\hline & & $10-16-91$ & USGS & $\mathrm{U}$ & 24.86 & $S$ & $S$ \\
\hline \multirow[t]{2}{*}{$52 S 21$} & $377 \mathrm{CPMC}$ & $05-15-91$ & USGS & $\mathrm{U}$ & 16.21 & $S$ & $S$ \\
\hline & & $10-16-91$ & USGS & $\mathrm{U}$ & 24.55 & $S$ & $S$ \\
\hline \multirow[t]{2}{*}{$52 \mathrm{~S} 24$} & 361QNTC & $05-14-91$ & USGS & 48 & 67.59 & $S$ & $\mathrm{~S}$ \\
\hline & & $10-16-91$ & USGS & $\mathrm{U}$ & 68.84 & $S$ & $S$ \\
\hline \multirow[t]{2}{*}{$52 S 26$} & 217РТМС & $05-14-91$ & USGS & $\mathrm{U}$ & 3.87 & $S$ & $S$ \\
\hline & & $10-16-91$ & USGS & $\mathrm{U}$ & -- & $\mathrm{D}$ & $S$ \\
\hline $52 S 29$ & 360LGRV & $05-15-91$ & USGS & $\mathrm{U}$ & -1.43 & $S$ & $S$ \\
\hline & & $10-16-91$ & USGS & $\mathrm{U}$ & 3.18 & $S$ & $S$ \\
\hline $52 \mathrm{~S} 46$ & 112CLMB & $03-25-91$ & USGS & $\mathrm{U}$ & 5.28 & $S$ & $S$ \\
\hline & & $10-18-91$ & USGS & $\mathrm{U}$ & 5.78 & $S$ & $S$ \\
\hline $52 S 47$ & 217РТМC & $05-16-91$ & USGS & $\mathbf{N}$ & 113.08 & $P$ & $S$ \\
\hline & & $10-15-91$ & USGS & $\mathrm{N}$ & 102.54 & $S$ & $S$ \\
\hline
\end{tabular}


Table 2. Synoptic water-level measurements for selected wells in Prince William County-Continued

[Depths are measured from land surface datum. For location of wells, refer to plate 1]

\begin{tabular}{|c|c|c|c|c|c|c|c|}
\hline $\begin{array}{l}\text { Local } \\
\text { well } \\
\text { number }\end{array}$ & $\begin{array}{c}\text { Geologic } \\
\text { unit }\end{array}$ & Date & Agency & $\begin{array}{c}\text { Recovery } \\
\text { period } \\
\text { (hours) }\end{array}$ & $\begin{array}{l}\text { Water } \\
\text { level } \\
\text { (feet) }\end{array}$ & $\begin{array}{c}\text { Site } \\
\text { status }\end{array}$ & $\begin{array}{l}\text { Method of } \\
\text { measurmen }\end{array}$ \\
\hline \multirow[t]{2}{*}{$52 \mathrm{~S} 48$} & 217PTMC & $05-21-91$ & USGS & $\mathrm{U}$ & 23.13 & $S$ & $S$ \\
\hline & & $10-16-91$ & USGS & $\mathrm{U}$ & 23.56 & $\mathrm{~S}$ & S \\
\hline \multirow[t]{2}{*}{$52 S 49$} & 217PTMC & $05-16-91$ & USGS & $\mathrm{N}$ & 121.20 & $\mathrm{~S}$ & $\mathrm{~S}$ \\
\hline & & $10-22-91$ & USGS & $N$ & 122.04 & $S$ & $S$ \\
\hline \multirow[t]{2}{*}{$52 \mathrm{~S} 50$} & 217PTMC & $05-21-91$ & USGS & 8.4 & 114.50 & $\mathrm{~S}$ & $\mathrm{~S}$ \\
\hline & & $10-17-91$ & USGS & 8.6 & 114.66 & $\mathrm{R}$ & $\mathrm{S}$ \\
\hline $52 \mathrm{~S} 51$ & 217PTMC & $10-25-91$ & USGS & $\mathrm{U}$ & 45.42 & S & $S$ \\
\hline $52 \mathrm{~S} 52$ & 217PTMC & $10-25-91$ & USGS & $\mathrm{N}$ & 69.51 & $\mathrm{~S}$ & $\mathrm{~s}$ \\
\hline \multirow[t]{2}{*}{$52 S 56$} & 217PTMC & $05-16-91$ & USGS & $\mathrm{U}$ & 14.33 & $\mathrm{~S}$ & $\mathrm{~S}$ \\
\hline & & $10-22-91$ & USGS & $\mathrm{U}$ & 14.89 & $\mathrm{~S}$ & $\mathrm{~S}$ \\
\hline \multirow[t]{2}{*}{$52 \mathrm{~S} 57$} & 217PTMC & $05-21-91$ & USGS & $\mathrm{U}$ & 4.05 & $\mathrm{~S}$ & $\mathrm{~S}$ \\
\hline & & $10-15-91$ & USGS & $\mathrm{U}$ & 5.74 & $S$ & $S$ \\
\hline \multirow[t]{2}{*}{$52 \mathrm{~T} 67$} & $3600 C C Q$ & $05-22-91$ & USGS & $\mathrm{U}$ & 54.56 & $\mathrm{~S}$ & $\mathrm{~S}$ \\
\hline & & $10-18-91$ & USGS & $\mathrm{U}$ & 57.90 & $\mathrm{~S}$ & $\mathrm{~s}$ \\
\hline \multirow[t]{2}{*}{$52 \mathrm{~T} 68$} & $3600 C C Q$ & $05-23-91$ & USGS & $\mathrm{U}$ & 8.74 & $\mathrm{~S}$ & $S$ \\
\hline & & $10-18-91$ & USGS & $\mathrm{U}$ & 25.83 & $S$ & $\mathrm{~S}$ \\
\hline \multirow[t]{2}{*}{$53 \mathrm{~S} 1$} & 217PTMC & $05-16-91$ & USGS & $\mathrm{U}$ & 2.50 & $\mathrm{~S}$ & $\mathrm{~S}$ \\
\hline & & $10-22-91$ & USGS & $\mathrm{U}$ & 3.30 & $S$ & $\mathrm{~S}$ \\
\hline \multirow[t]{2}{*}{$53 \mathrm{~T} 1$} & 217PTMC & $05-21-91$ & USGS & $\mathrm{U}$ & 2.05 & $\mathrm{~S}$ & S \\
\hline & & $10-15-91$ & USGS & $\mathrm{U}$ & 2.39 & $S$ & $S$ \\
\hline \multirow[t]{2}{*}{ 53T 2 SOW 029} & 217PTMC & $05-21-91$ & USGS & W & 2.01 & $S$ & $\mathrm{~S}$ \\
\hline & & $10-09-91$ & VWCB & W & 2.83 & $S$ & $S$ \\
\hline \multirow[t]{2}{*}{$53 \mathrm{~T} 55$} & 361QNTC & $05-21-91$ & USGS & $\mathrm{N}$ & 26.08 & $\mathrm{~S}$ & $S$ \\
\hline & & $10-15-91$ & USGS & $\mathrm{N}$ & 27.29 & $\mathbf{R}$ & $S$ \\
\hline \multirow[t]{2}{*}{ 53T 57} & $112 \mathrm{CLMB}$ & $05-16-91$ & USGS & $\mathrm{U}$ & 4.67 & $S$ & $S$ \\
\hline & & $10-16-91$ & USGS & $\mathrm{U}$ & 4.77 & $\mathrm{~S}$ & $S$ \\
\hline
\end{tabular}




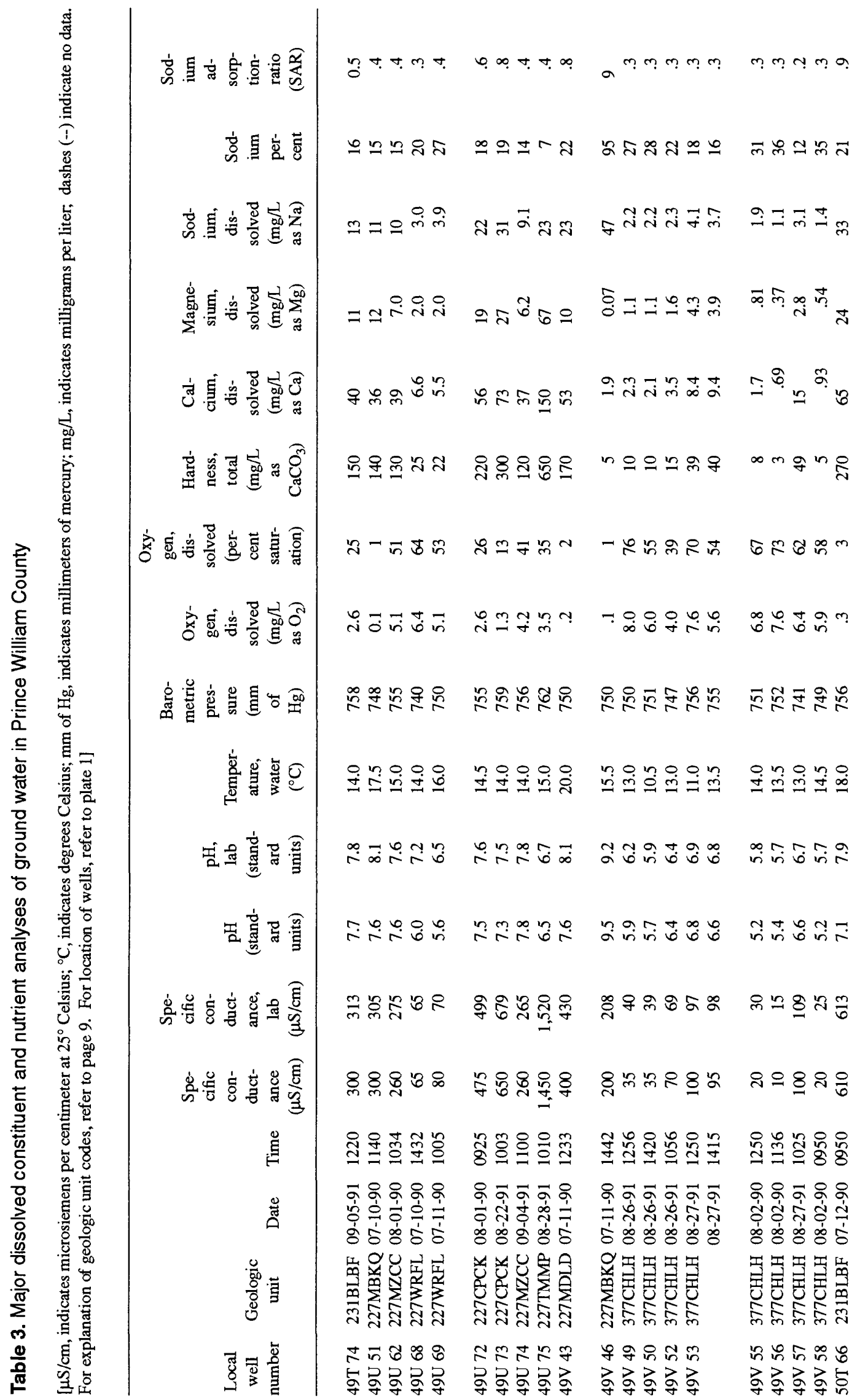




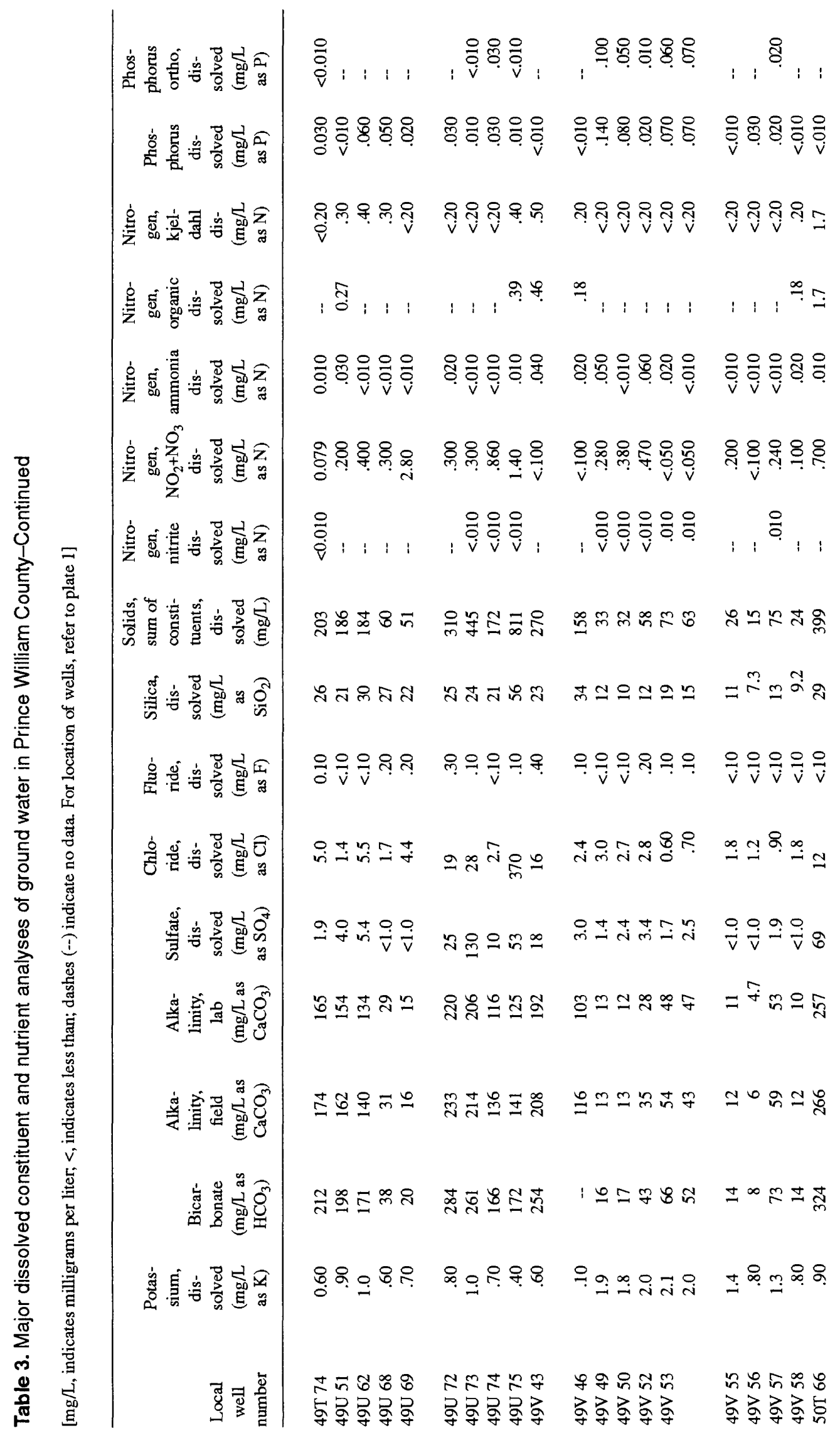




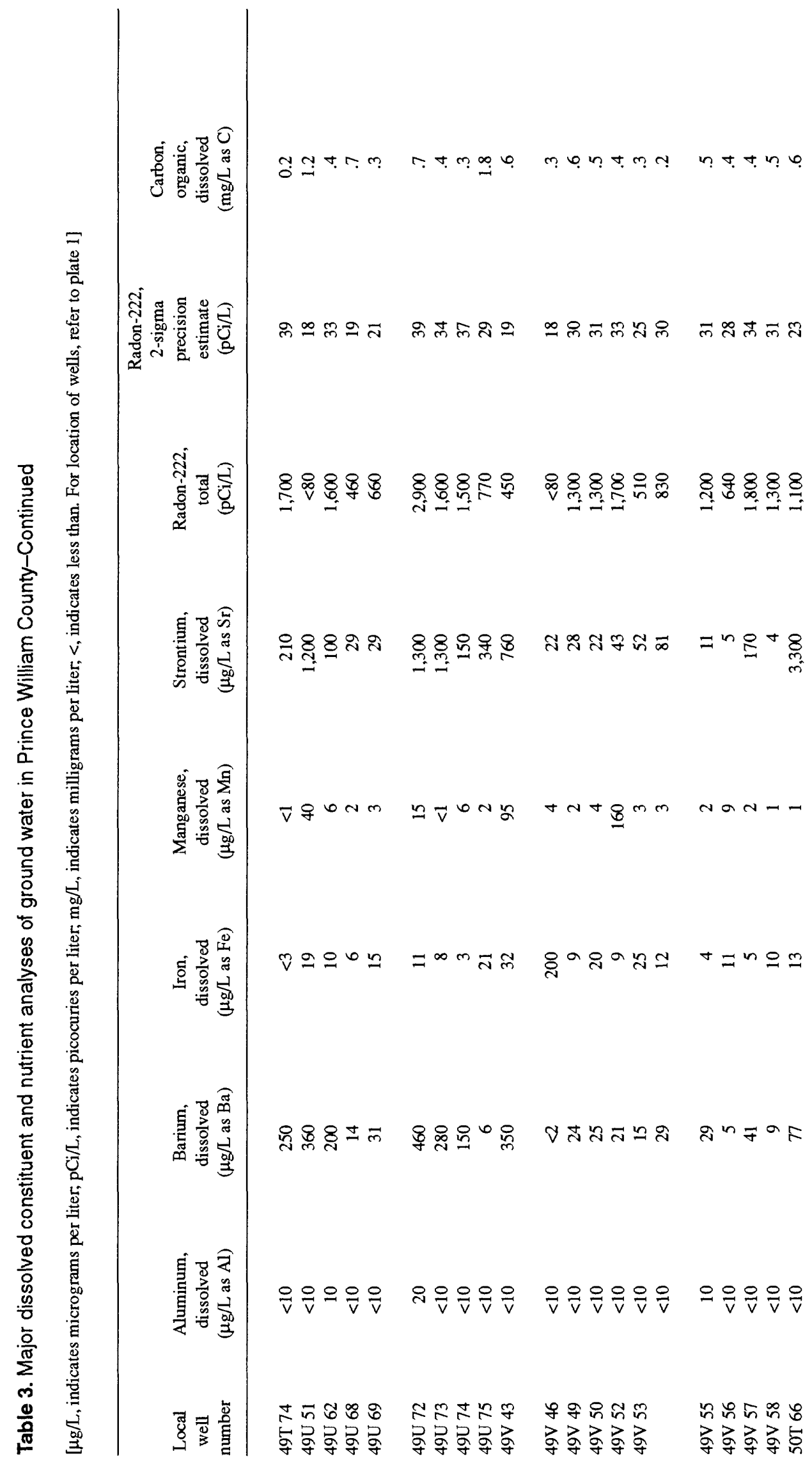




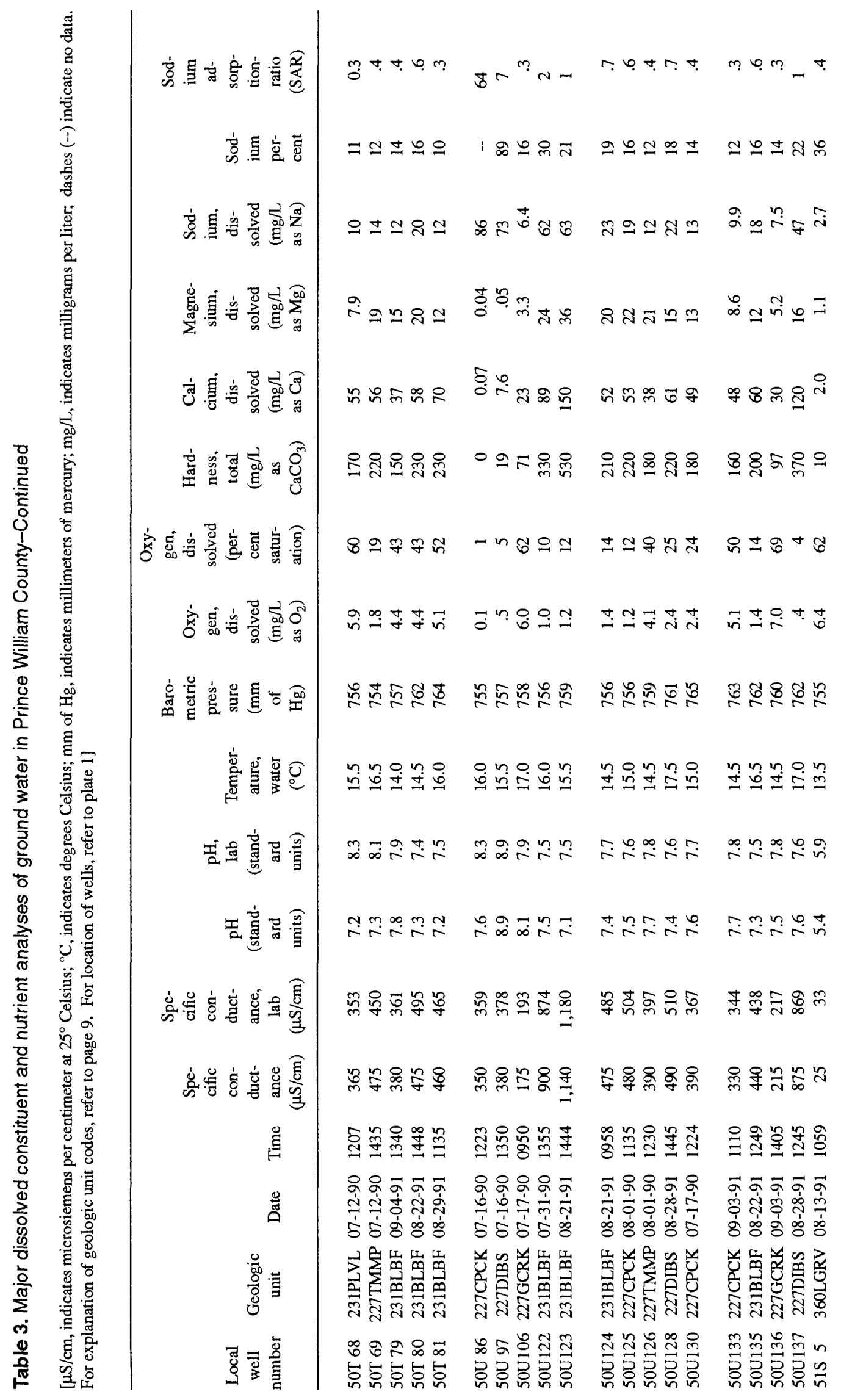




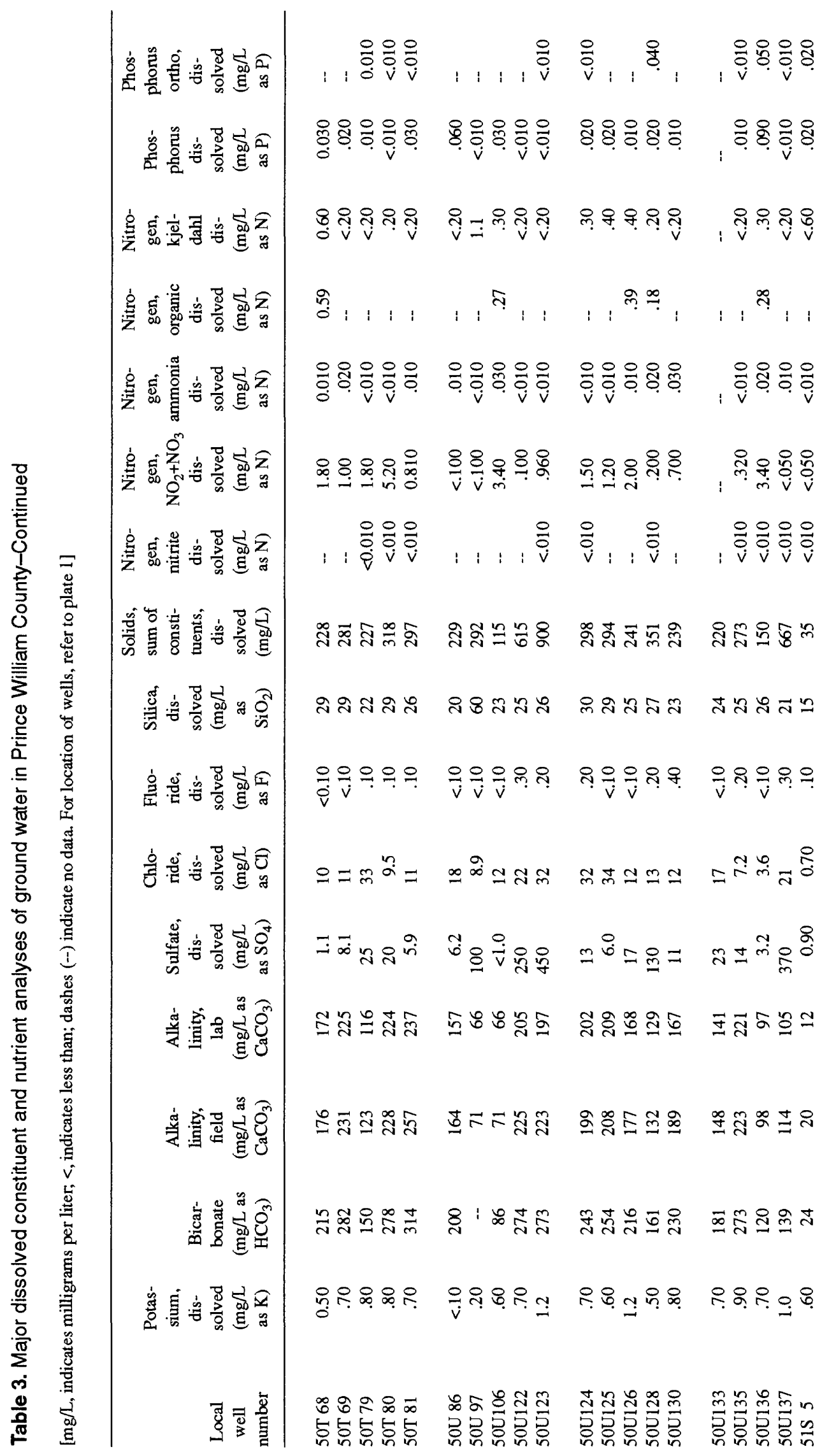




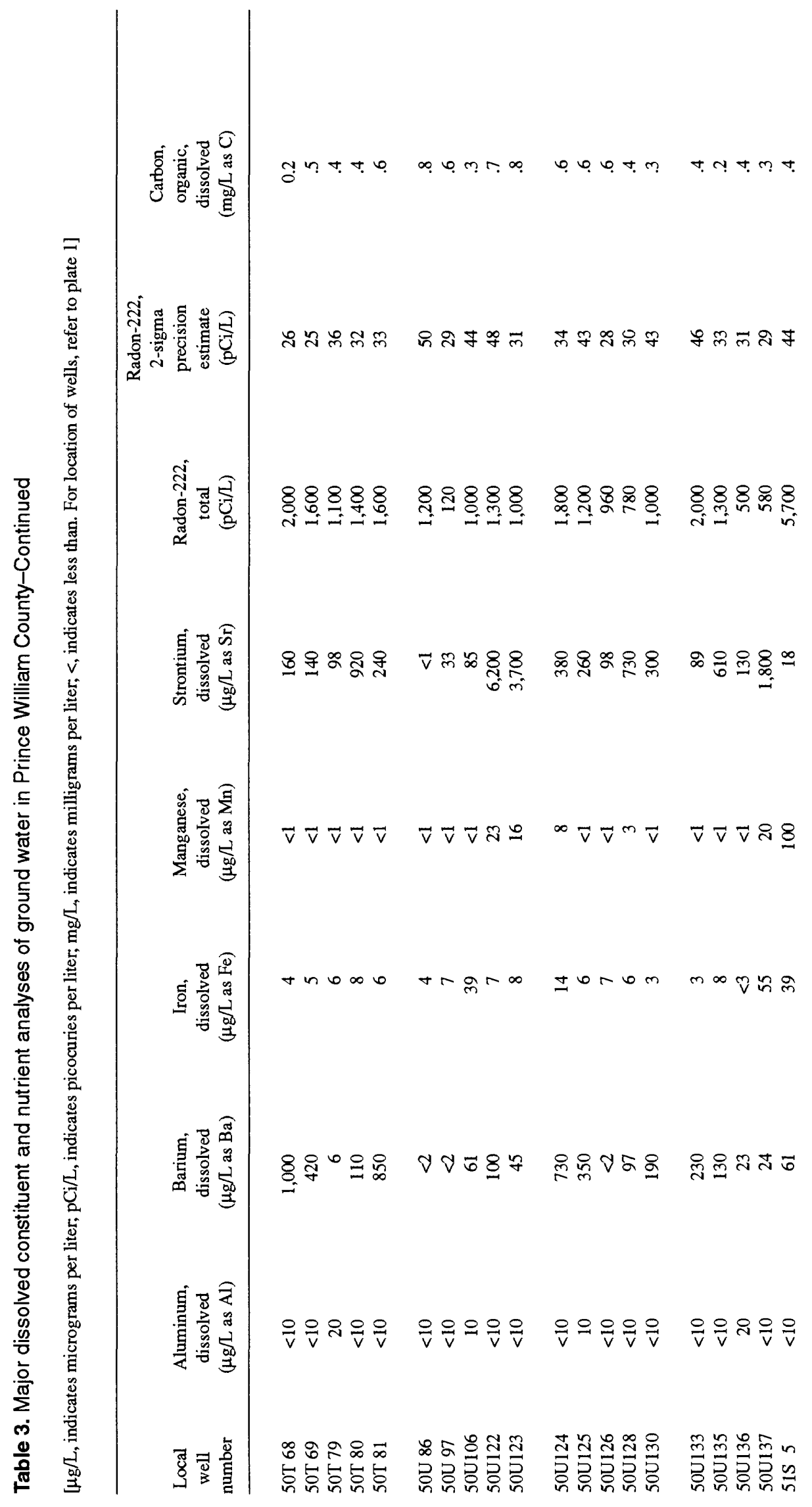




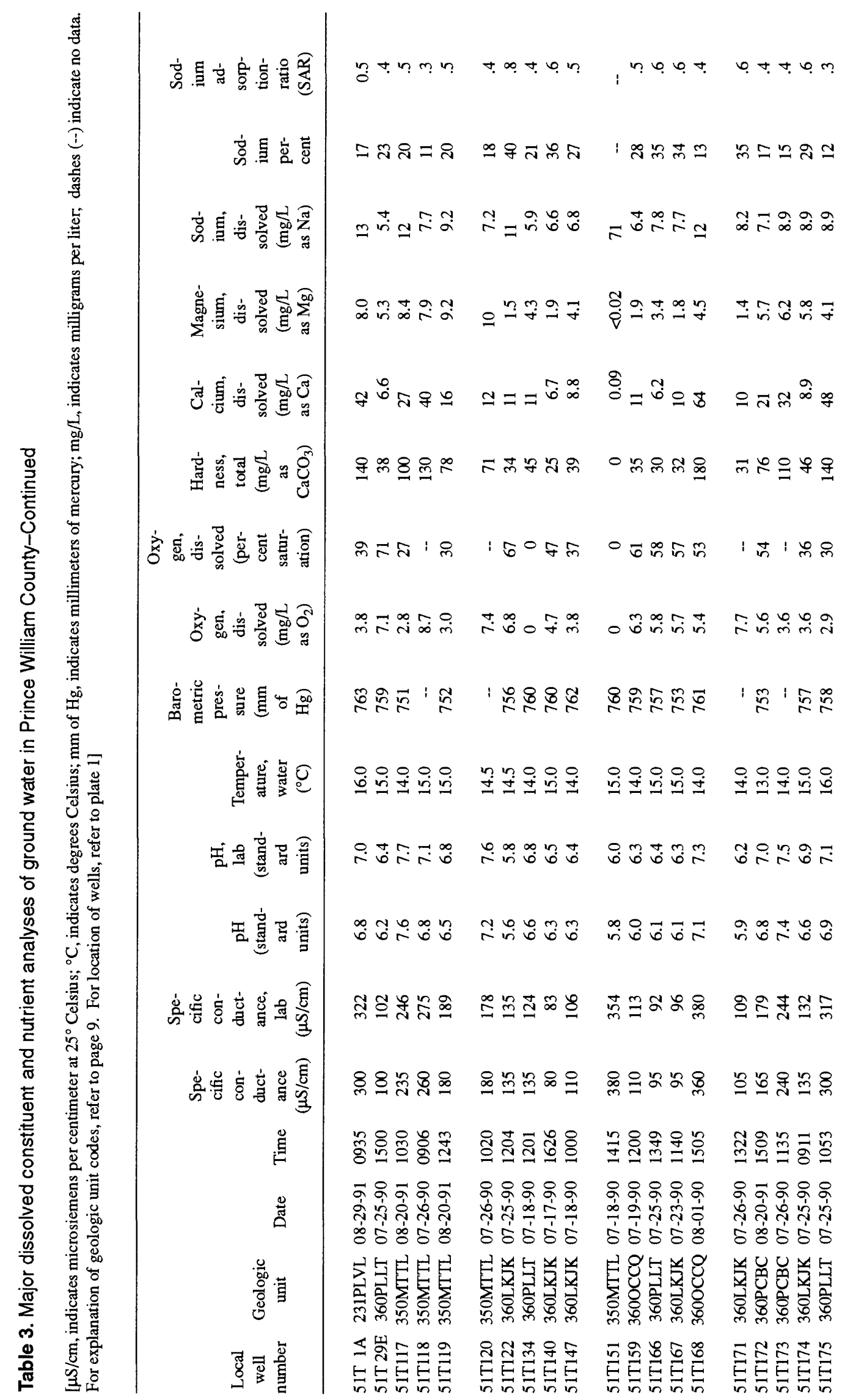




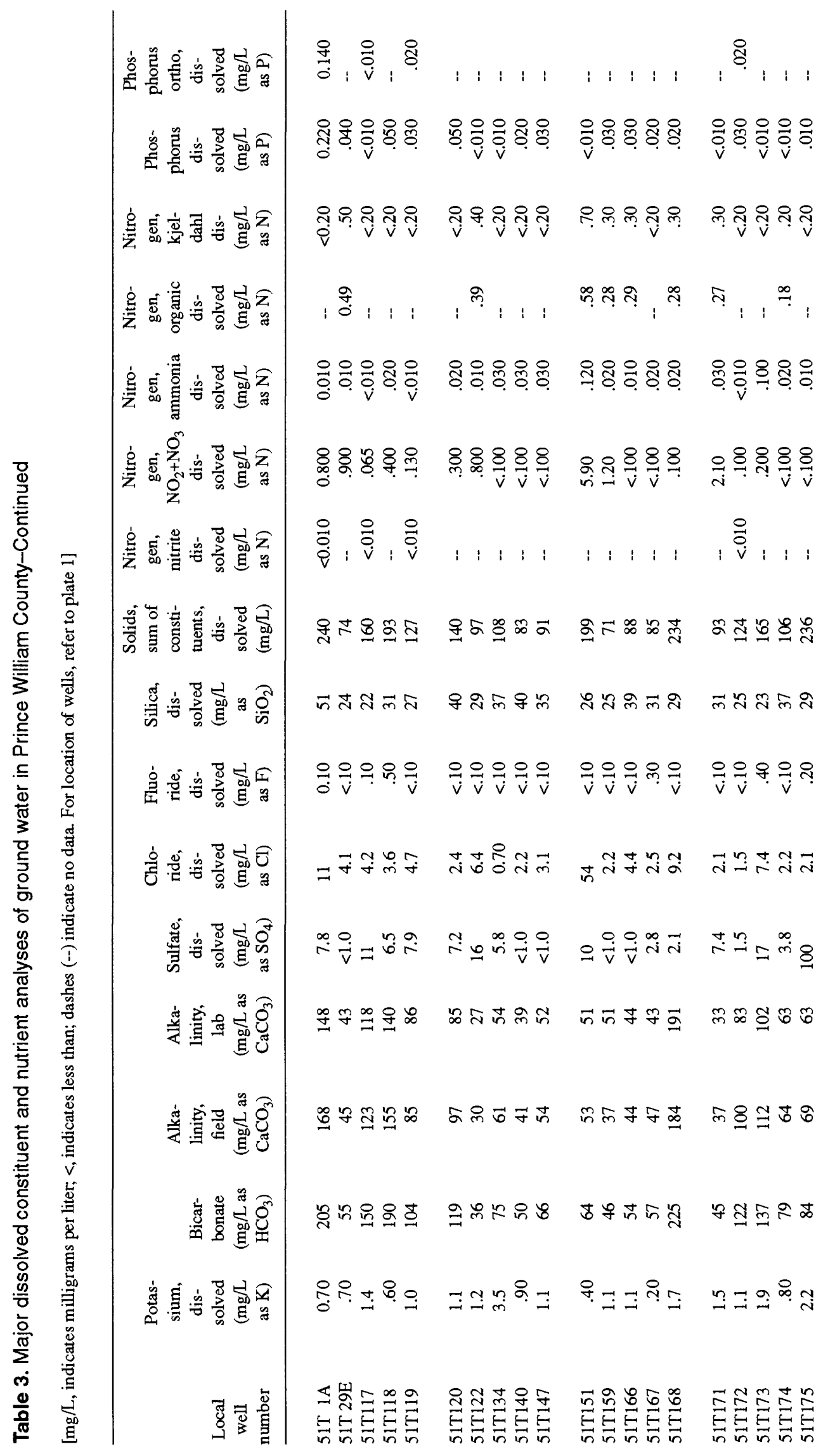




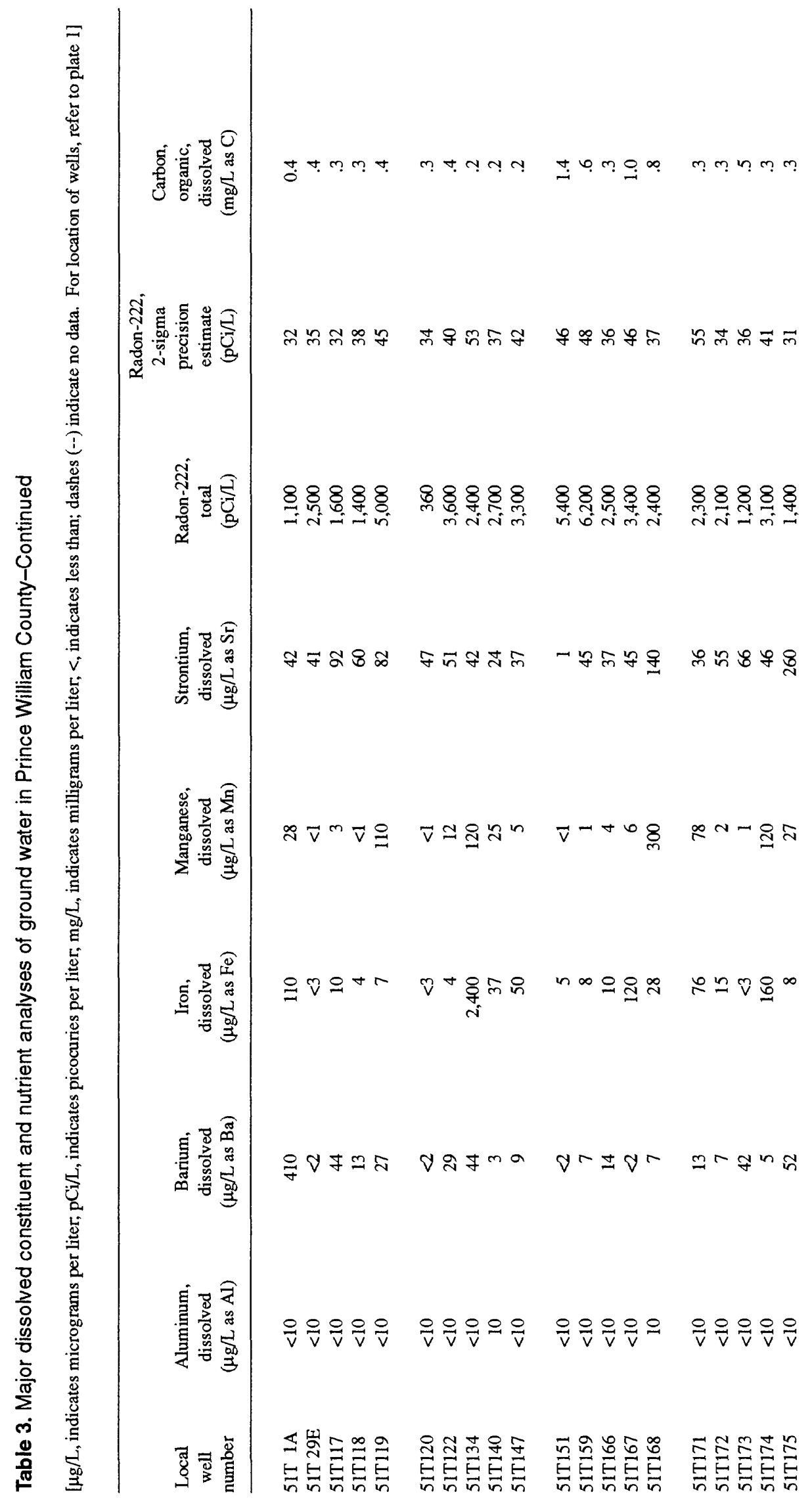




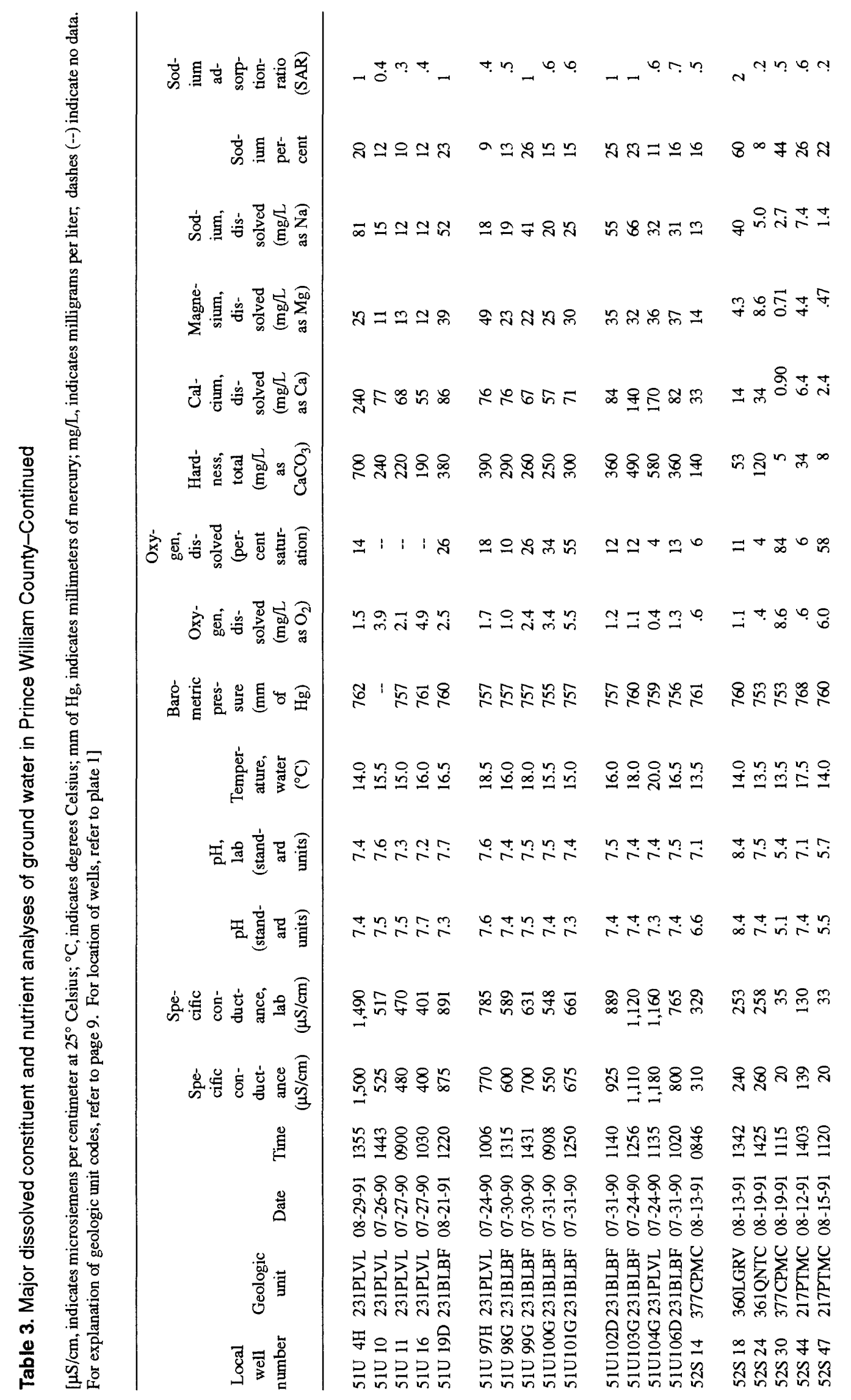




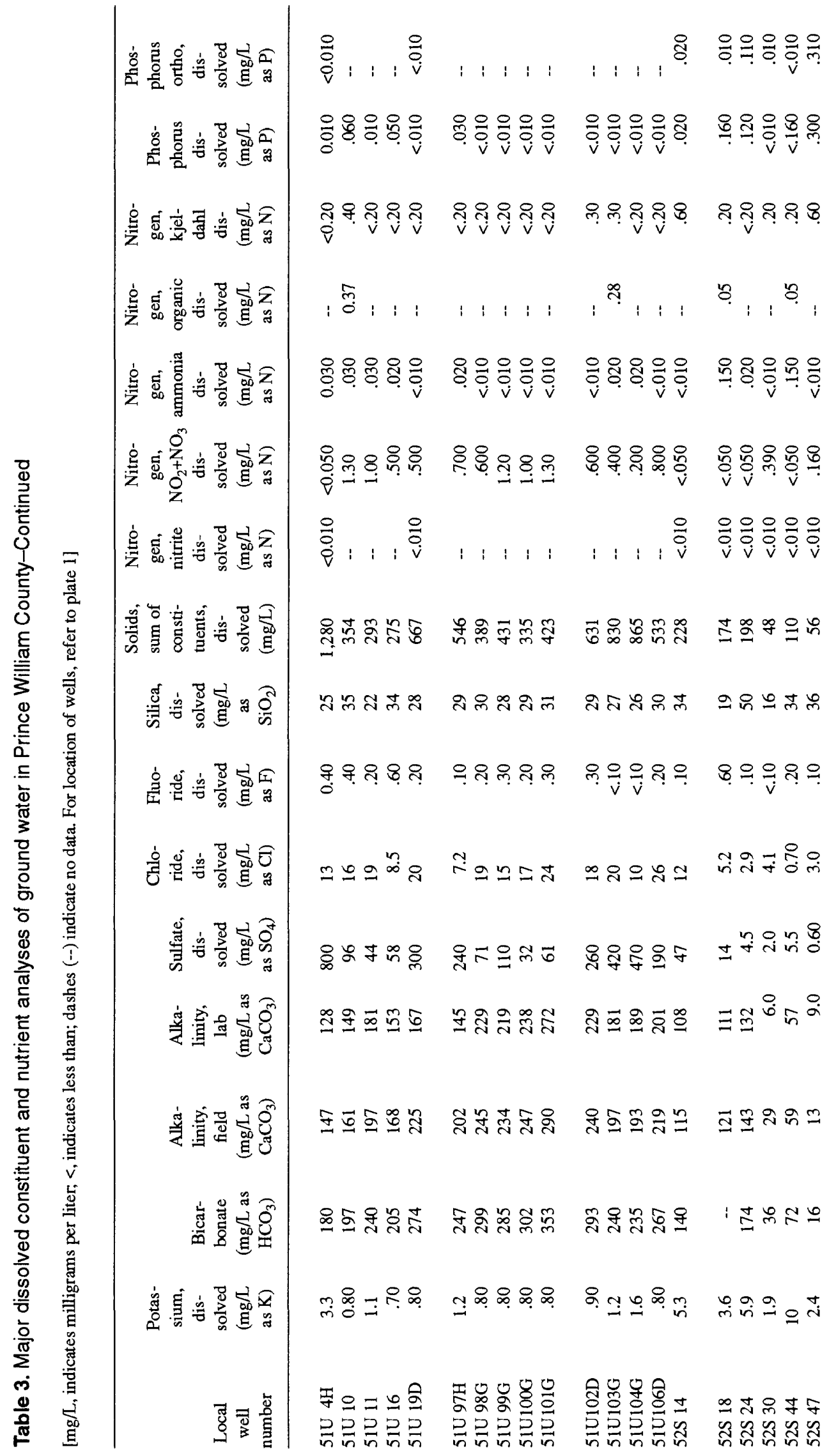




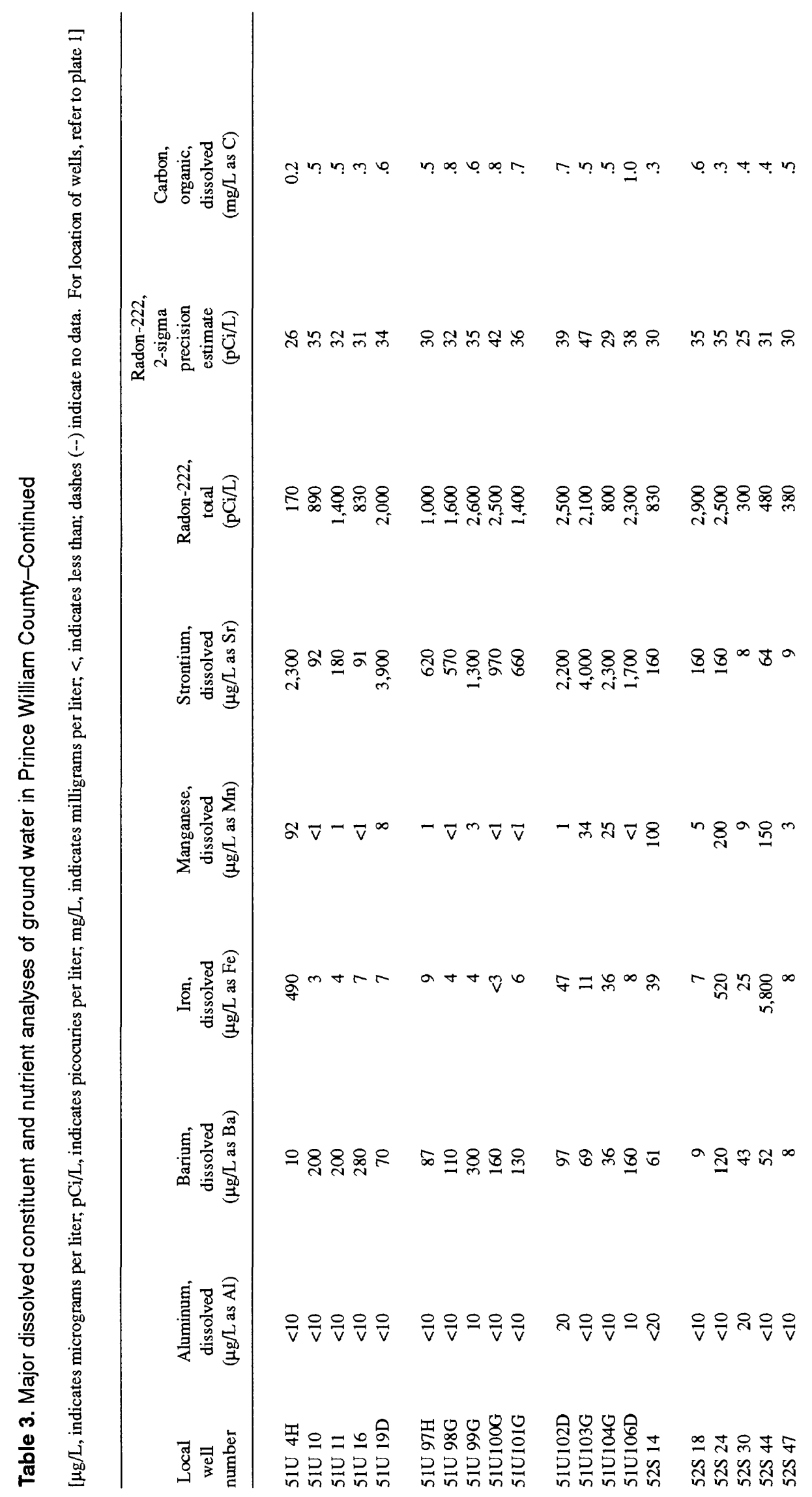




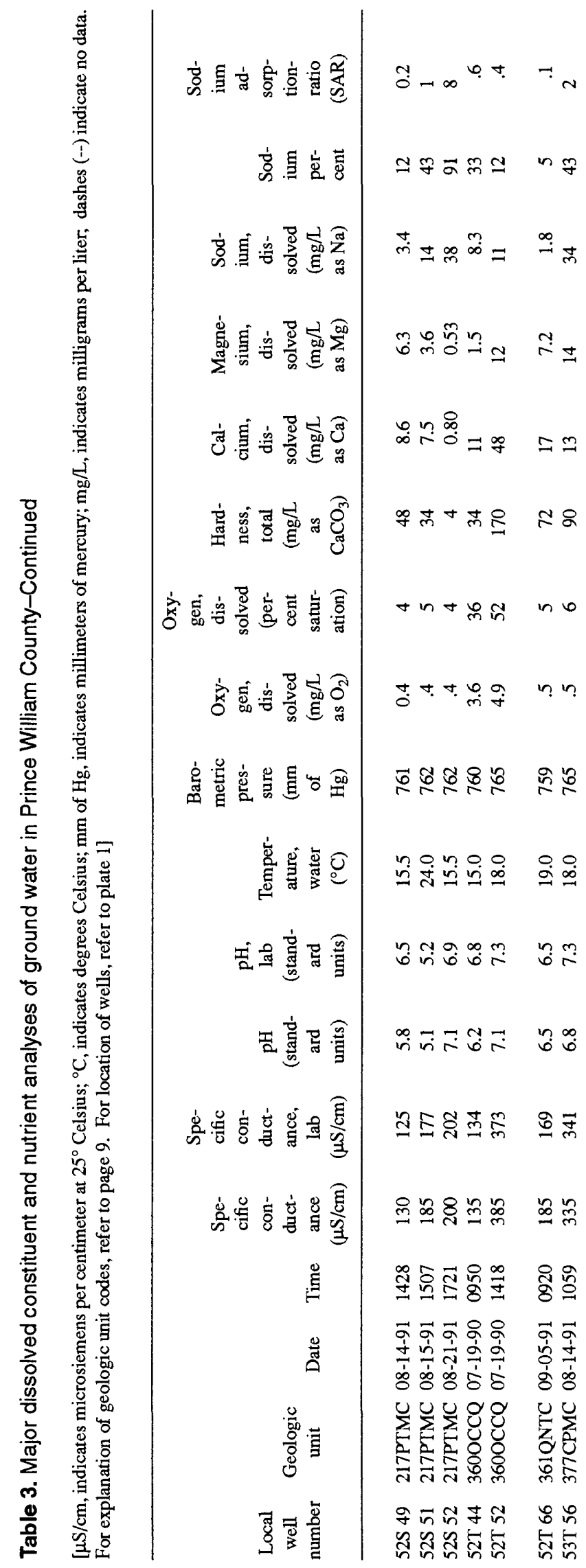




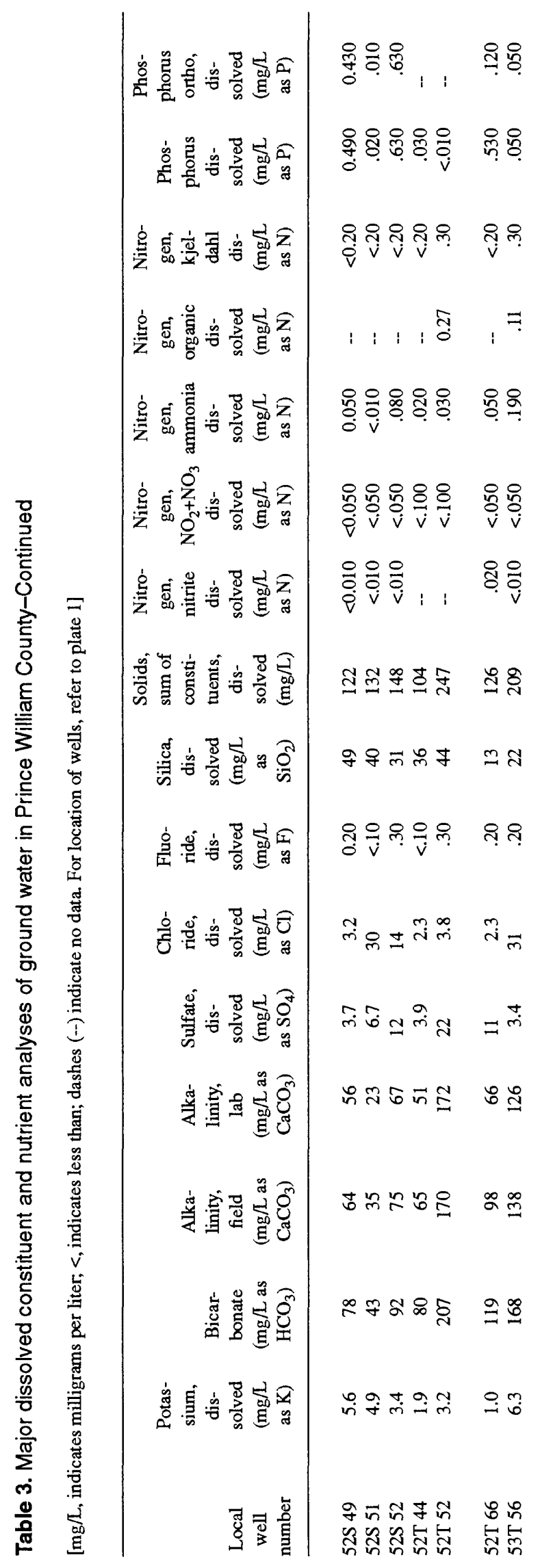




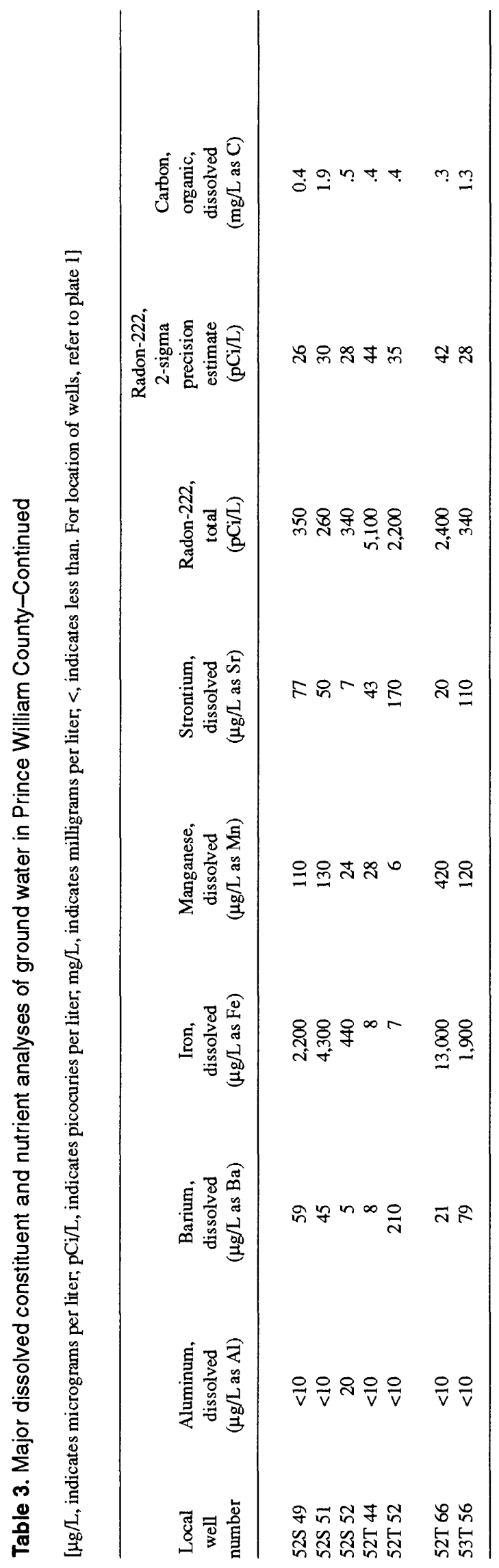




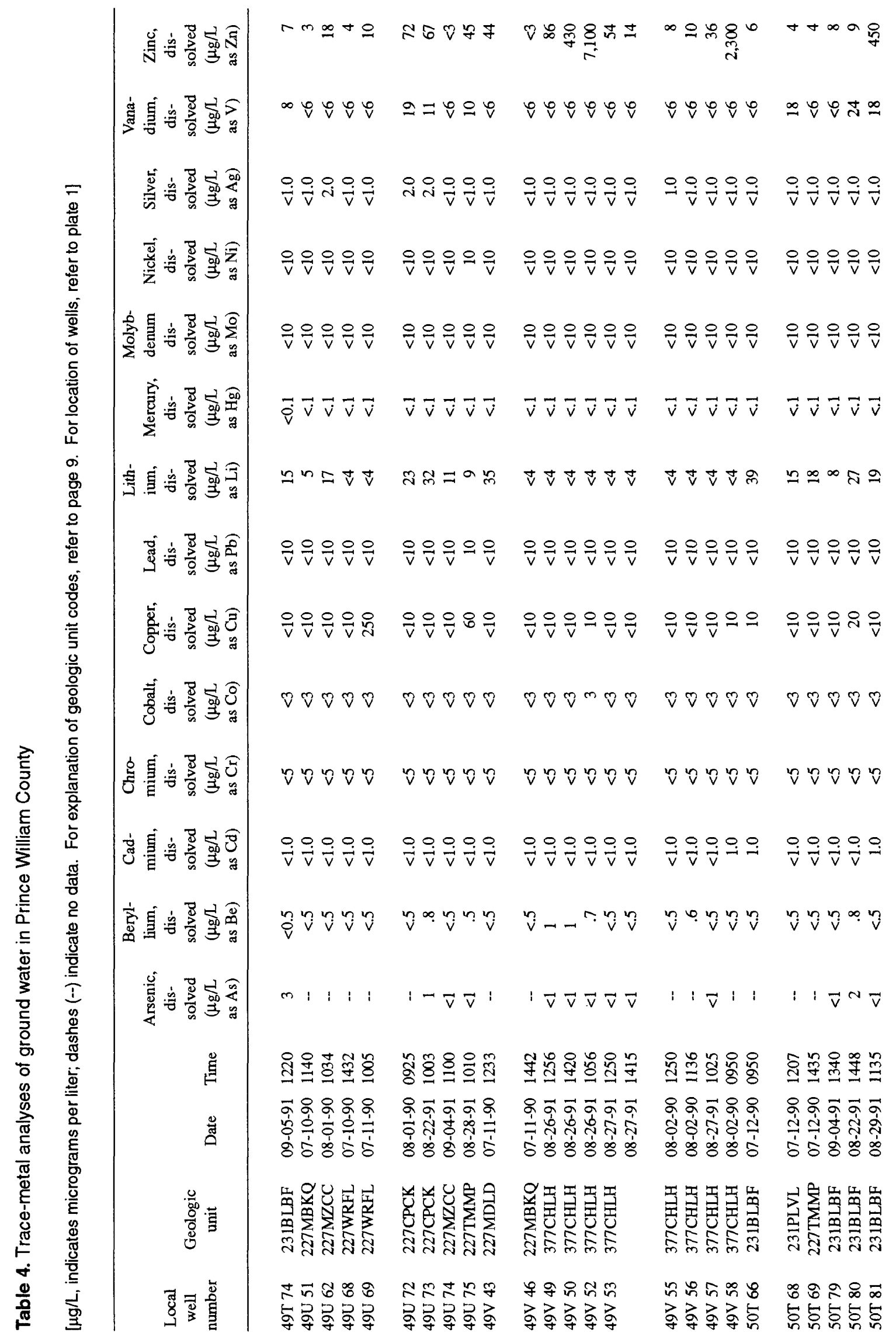




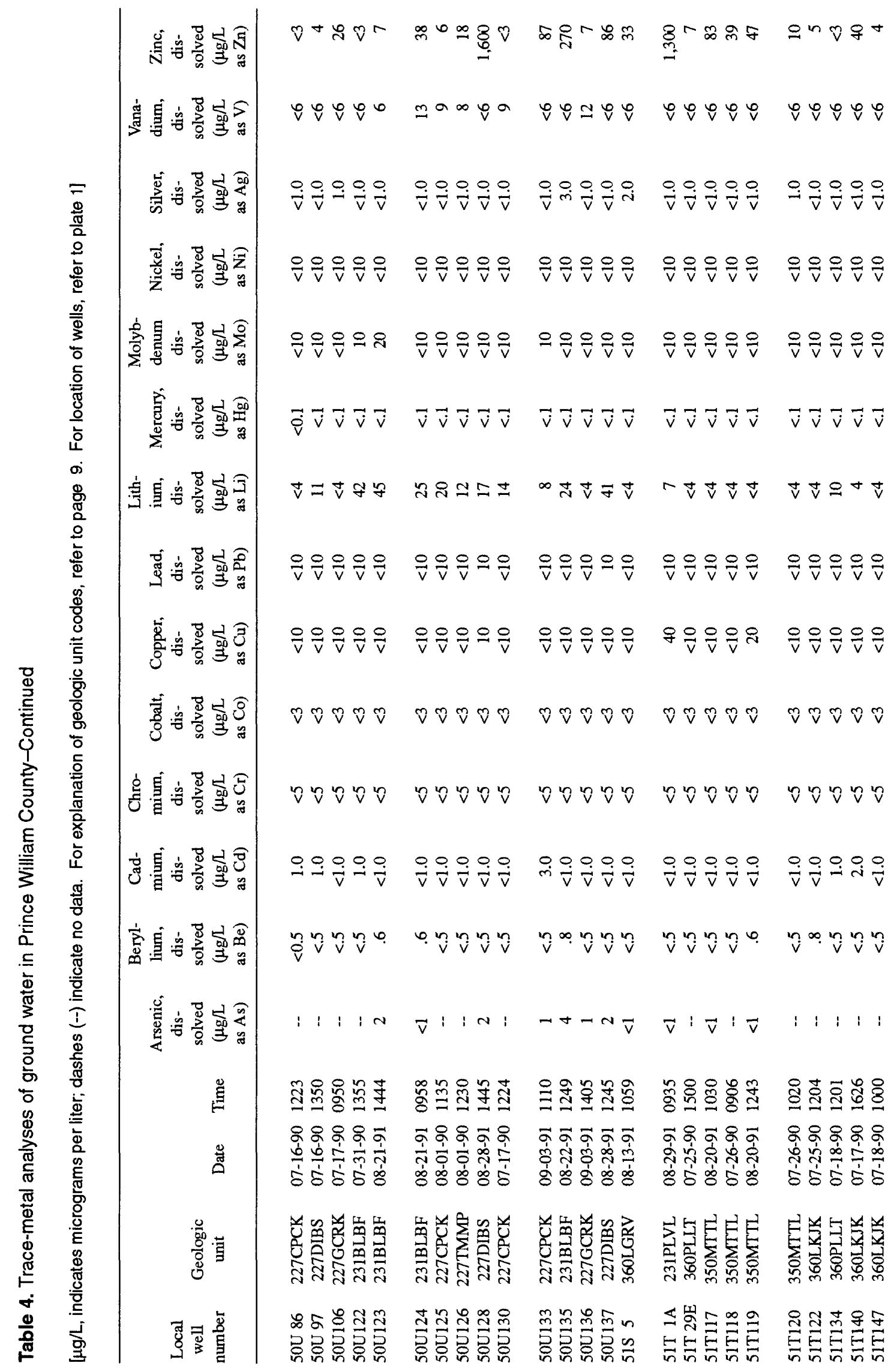




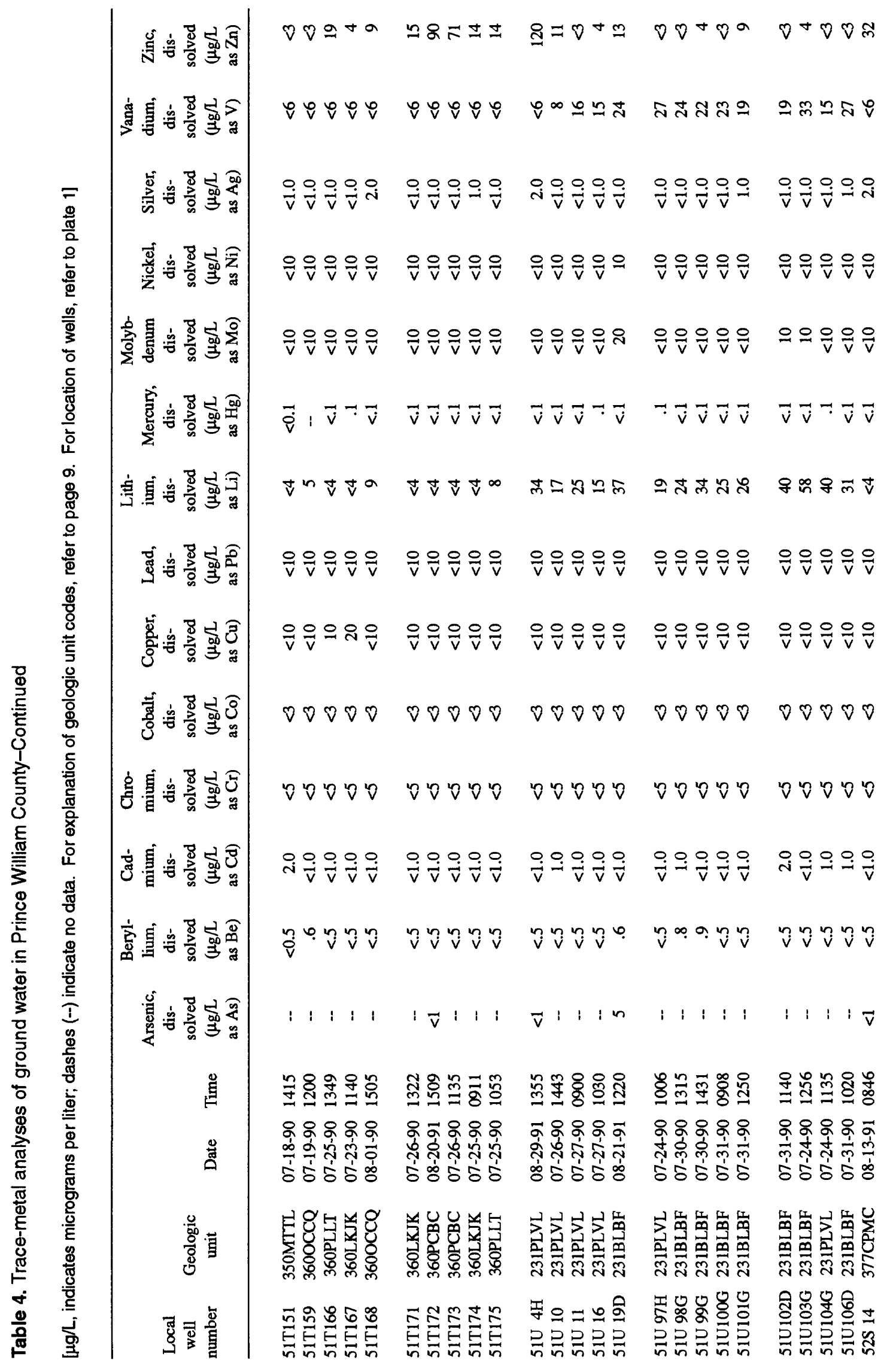




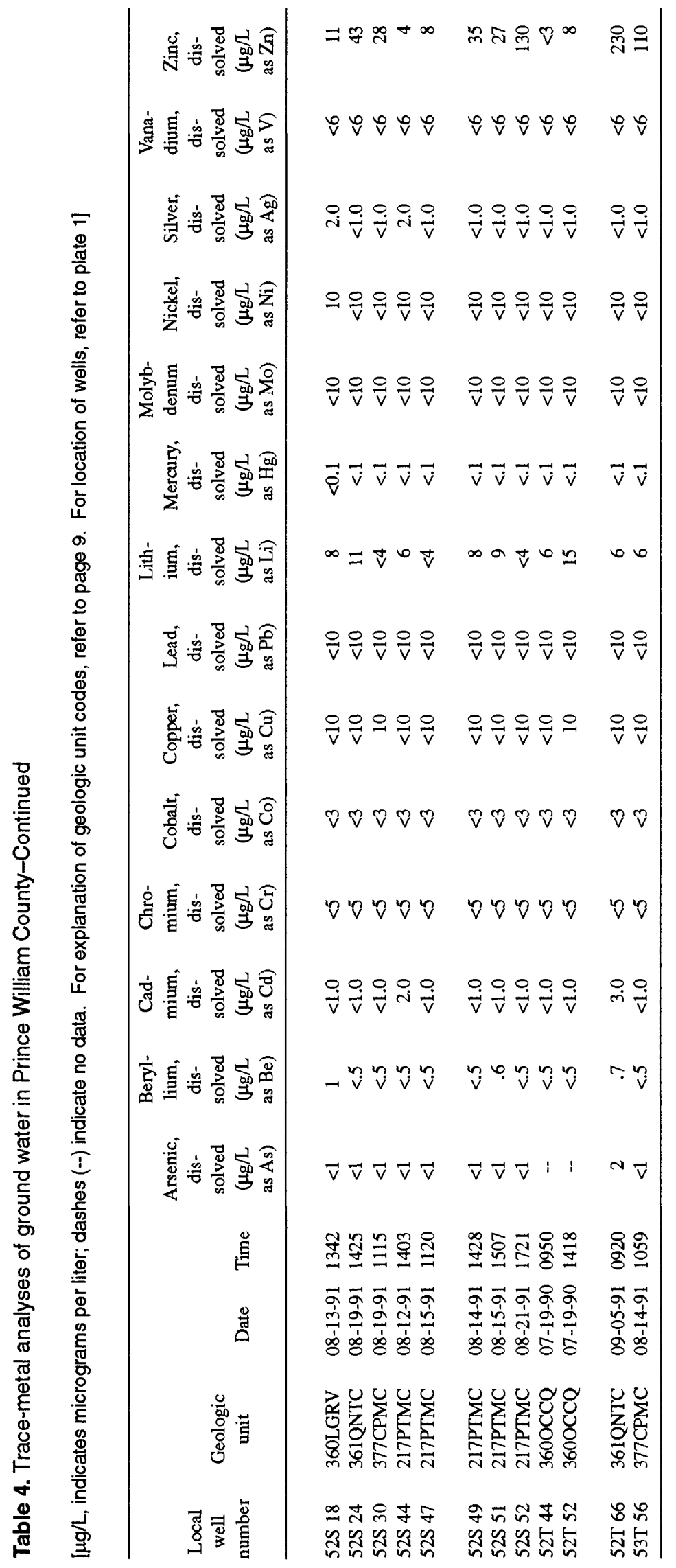


Table 5. Environmental isotope analyses of ground water in Prince William County

$[\mathrm{pCi} / \mathrm{L}$, picocuries per liter; TU, tritium units; dashes (--) indicate no data. H-2/H-1 and O-18/O-16 ratios are reported relative to Vienna Standard Mean Ocean Water (VSMOW). For explanation of geologic unit codes, refer to page 9. For location of wells, refer to plate 1]

\begin{tabular}{|c|c|c|c|c|c|c|c|c|}
\hline $\begin{array}{l}\text { Local } \\
\text { well } \\
\text { number }\end{array}$ & $\begin{array}{l}\text { Geologic } \\
\text { unit }\end{array}$ & Date & Time & $\begin{array}{c}\text { Tritium, } \\
\text { total } \\
(\mathrm{pCi} / \mathrm{L})\end{array}$ & $\begin{array}{c}\text { Tritium, } \\
2 \text { sigma } \\
\text { precision } \\
\text { estimate } \\
(\mathrm{pCi} / \mathrm{L})\end{array}$ & $\begin{array}{c}\text { Tritium, } \\
\text { total } \\
\text { (TU) }\end{array}$ & $\begin{array}{l}\text { H-2/H-1 } \\
\text { stable } \\
\text { isotope } \\
\text { ratio } \\
\text { (per mil) }\end{array}$ & $\begin{array}{c}\text { O-18/O-16 } \\
\text { stable } \\
\text { isotope } \\
\text { ratio } \\
\text { (per mil) }\end{array}$ \\
\hline $49 \mathrm{~T} 74$ & 231BLBF & $09-05-91$ & 1220 & -0.3 & 1.9 & -0.1 & -44 & -7.4 \\
\hline $49 U 73$ & $227 \mathrm{CPCK}$ & $08-22-91$ & 1003 & 2.2 & 3.2 & .7 & -42 & -7.25 \\
\hline $49 \cup 74$ & $227 \mathrm{MZCC}$ & $09-04-91$ & 1100 & 6.4 & 2.6 & 2 & -46.5 & -7.7 \\
\hline $49 U 75$ & 227TMMP & $08-28-91$ & 1010 & 65 & 5.8 & 20.2 & -45 & -7.35 \\
\hline $49 \mathrm{~V} 49$ & 377CHLH & $08-26-91$ & 1256 & 59 & 4.5 & 18.5 & -47.5 & -8.05 \\
\hline $49 \mathrm{~V} 50$ & $377 \mathrm{CHLH}$ & $08-26-91$ & 1420 & 63 & 4.4 & 19.6 & -46.5 & -8.05 \\
\hline $49 \vee 52$ & 377CHLH & $08-26-91$ & 1056 & 51 & 4.4 & 16.1 & -48 & -8.15 \\
\hline $49 \vee 53$ & 377CHLH & $08-27-91$ & 1250 & 8.6 & 2.6 & 2.7 & -47.5 & -8 \\
\hline & & $08-27-91$ & 1415 & 14 & 2.6 & 4.3 & -48 & -7.95 \\
\hline $49 \vee 55$ & 377CHLH & $02-04-91$ & 1344 & 45 & 4 & 14.2 & -47.5 & -8.05 \\
\hline $49 V 56$ & 377CHLH & $02-04-91$ & 1252 & 46 & 3 & 14.4 & -49.5 & -8.15 \\
\hline 49V 57 & 377CHLH & $08-27-91$ & 1025 & 44 & 4.4 & 13.9 & -48.5 & -8.15 \\
\hline 49V 58 & 377CHLH & $02-04-91$ & 1105 & 21 & 2 & 6.6 & -49 & -8.1 \\
\hline 50T 79 & 231BLBF & $09-04-91$ & 1340 & 60 & 4.4 & 18.9 & -43 & -7.4 \\
\hline $50 \mathrm{~T} 80$ & 231BLBF & $08-22-91$ & 1448 & 3.8 & 1.9 & 1.2 & -43 & -7.2 \\
\hline $50 \mathrm{~T} 81$ & 231BLBF & $08-29-91$ & 1135 & 17 & 2.6 & 5.3 & -43.5 & -7.45 \\
\hline $50 \mathrm{U} 122$ & 231BLBF & $09-11-91$ & 1240 & 33 & 3.8 & 10.2 & -42.5 & -7.15 \\
\hline $50 \mathrm{U} 123$ & 231BLBF & $08-21-91$ & 1444 & 27 & 3.2 & 8.3 & -41.5 & -7.15 \\
\hline 50U124 & 231BLBF & $08-21-91$ & 0958 & 15 & 2.6 & 4.6 & -42.5 & -7.25 \\
\hline $50 \mathrm{U} 128$ & 227DIBS & $08-28-91$ & 1445 & 5.1 & 1.9 & 1.6 & -44.5 & -7.45 \\
\hline $50 U 133$ & 227CPCK & $09-03-91$ & 1110 & 22 & 2.6 & 7 & -45 & -7.6 \\
\hline 50U135 & 231BLBF & $08-22-91$ & 1249 & 3.2 & 1.9 & 1 & -44 & -7.3 \\
\hline $50 \mathrm{U} 136$ & 227GCRK & $09-03-91$ & 1405 & 64 & 4.4 & 19.9 & -44.5 & -7.75 \\
\hline $50 \mathrm{U} 137$ & 227DIBS & $08-28-91$ & 1245 & 0 & 1.9 & 0 & -41 & -7.15 \\
\hline $51 S 5$ & 360LGRV & $08-13-91$ & 1059 & 52 & 4.4 & 16.4 & -47 & -7.8 \\
\hline $51 \mathrm{~T} 1 \mathrm{~A}$ & 231PLVL & $08-29-91$ & 0935 & 22 & 2.6 & 6.9 & -44 & -7.35 \\
\hline $51 \mathrm{~T} 29 \mathrm{E}$ & 360PLLT & $02-05-91$ & 1645 & 77 & 6 & 23.9 & -45.5 & -7.55 \\
\hline $51 \mathrm{~T} 117$ & 350MTTL & $08-20-91$ & 1030 & 25 & 2.6 & 7.8 & -44 & -7.5 \\
\hline $51 T 118$ & 350MTTL & $02-05-91$ & 1421 & 57 & 5 & 17.8 & -45 & -7.65 \\
\hline $51 T 119$ & 350MTTL & $08-20-91$ & 1243 & 45 & 3.8 & 14 & -44.5 & -7.45 \\
\hline $51 T 167$ & 360LKJK & $09-11-91$ & 1500 & 72 & 5.1 & 22.6 & -. & -- \\
\hline $51 \mathrm{~T} 172$ & $360 \mathrm{PCBC}$ & $08-20-91$ & 1509 & 64 & 5.1 & 20.1 & -45 & -7.7 \\
\hline $51 T 173$ & $360 \mathrm{PCBC}$ & $02-05-91$ & 1516 & 58 & 5 & 18.1 & -42.5 & -7.3 \\
\hline $51 \mathrm{U} 4 \mathrm{H}$ & 231PLVL & $08-29-91$ & 1355 & -.6 & 1.9 & -.2 & -43 & -7.40 \\
\hline 51010 & 231PLVL & $02-04-91$ & 1545 & 35 & 3 & 10.8 & -45.5 & -7.35 \\
\hline $51 \mathrm{U} 11$ & 231PLVL & $09-10-91$ & 1130 & 28 & 3.2 & 8.8 & -43.5 & -7.45 \\
\hline $51 \mathrm{U} 16$ & 231PLVL & $09-10-91$ & 0955 & 6.4 & 2.6 & 2 & -42 & -7.4 \\
\hline 51U 19D & 231BLBF & $08-21-91$ & 1220 & 17 & 2.6 & 5.4 & -42.5 & -7.15 \\
\hline $51 \mathrm{U} 97 \mathrm{H}$ & 231PLVL & $02-05-91$ & 0943 & 17 & 2 & 5.3 & -44 & -7.15 \\
\hline 51U 98G & 231BLBF & $09-09-91$ & 1345 & 32 & 3.2 & 10.1 & -40.5 & -7 \\
\hline
\end{tabular}


Table 5. Environmental isotope analyses of ground water in Prince William County-Continued

[pCi/L, picocuries per liter; TU, tritium units; dashes (--) indicate no data. $\mathrm{H}-2 / \mathrm{H}-1$ and $\mathrm{O}-18 / \mathrm{O}-16$ ratios are reported relative to Vienna Standard Mean Ocean Water (VSMOW). For explanation of geologic unit codes, refer to page 9. For location of wells, refer to plate 1]

\begin{tabular}{|c|c|c|c|c|c|c|c|c|}
\hline $\begin{array}{l}\text { Local } \\
\text { well } \\
\text { number }\end{array}$ & $\begin{array}{l}\text { Geologic } \\
\text { unit }\end{array}$ & Date & Time & $\begin{array}{l}\text { Tritium, } \\
\text { total } \\
\text { (pCi/L) }\end{array}$ & $\begin{array}{c}\text { Tritium, } \\
2 \text { sigma } \\
\text { precision } \\
\text { estimate } \\
(\mathrm{pCi} / \mathrm{L})\end{array}$ & $\begin{array}{c}\text { Tritium, } \\
\text { total } \\
\text { (TU) }\end{array}$ & $\begin{array}{c}\text { H-2/H-1 } \\
\text { stable } \\
\text { isotope } \\
\text { ratio } \\
\text { (per mil) }\end{array}$ & $\begin{array}{c}\text { O-18/O-16 } \\
\text { stable } \\
\text { isotope } \\
\text { ratio } \\
\text { (per mil) }\end{array}$ \\
\hline $51 \mathrm{U} 99 \mathrm{G}$ & 231BLBF & 09-09-91 & 1120 & 14 & 2.6 & 4.3 & -41.5 & -7.25 \\
\hline $51 \mathrm{U} 100 \mathrm{G}$ & 231BLBF & $09-10-91$ & 1425 & 28 & 3.2 & 8.8 & -41 & -7 \\
\hline $51 \mathrm{U} 101 \mathrm{G}$ & 231BLBF & $09-10-91$ & 1305 & 23 & 2.6 & 7.1 & -42.5 & -7.1 \\
\hline $51 U 102 \mathrm{D}$ & 231BLBF & $09-11-91$ & 1110 & 30 & 3.2 & 9.4 & -40.5 & -6.85 \\
\hline 51U103G & 231BLBF & $02-05-91$ & 1142 & 19 & 2 & 5.9 & -43 & -7.15 \\
\hline 51U104G & 231PLVL & $02-05-91$ & 1035 & 22 & 3 & 7 & -42.5 & -7.2 \\
\hline 51U106D & 231BLBF & $09-11-91$ & 0945 & 34 & 3.2 & 10.5 & -40.5 & -6.8 \\
\hline $52 S 14$ & 377CPMC & $08-13-91$ & 0846 & 38 & 3.8 & 11.8 & -42 & -7.35 \\
\hline $52 S 18$ & 360LGRV & $08-13-91$ & 1342 & 11 & 2.6 & 3.5 & -40.5 & -7 \\
\hline $52 S 24$ & 361QNTC & $08-19-91$ & 1425 & 2.9 & 1.9 & 0.9 & -39.5 & -7 \\
\hline 52530 & 377CPMC & $08-19-91$ & 1115 & 68 & 5.8 & 21.1 & -44 & -7.45 \\
\hline $52 \mathrm{~S} 44$ & 217PTMC & $08-12-91$ & 1403 & 2.6 & 2.6 & .8 & -41 & -7.15 \\
\hline $52 \mathrm{~S} 47$ & 217PTMC & $08-15-91$ & 1120 & 16 & 2.6 & 5.1 & -43.5 & -7.35 \\
\hline $52 S 49$ & 217PTMC & $08-14-91$ & 1428 & 0.6 & 2.6 & .2 & -40 & -6.9 \\
\hline $52 S 51$ & 217PTMC & $08-15-91$ & 1507 & 75 & 5.1 & 23.3 & -39.5 & -6.45 \\
\hline $52 S 52$ & 217PTMC & $08-21-91$ & 1721 & .6 & 2.6 & .2 & -46 & -7.7 \\
\hline $52 \mathrm{~T} 66$ & 361QNTC & $09-05-91$ & 0920 & 44 & 3.8 & 13.7 & -43 & -7.5 \\
\hline 53T 56 & 377СРМС & $08-14-91$ & 1059 & 19 & 2.6 & 6 & -41 & -6.9 \\
\hline
\end{tabular}


Table 6. Dissolved gas analyses of ground water in Prince William County

[mg/L, indicates milligrams per liter. For explanation of geologic unit codes, refer to page 9. For location of wells, refer to plate 1]

\begin{tabular}{|c|c|c|c|c|c|c|c|c|c|}
\hline $\begin{array}{l}\text { Local } \\
\text { well } \\
\text { number }\end{array}$ & $\begin{array}{c}\text { Geologic } \\
\text { unit }\end{array}$ & Date & $\begin{array}{c}\text { Argon } \\
(\mathrm{mg} / \mathrm{L} \text { as } \\
\mathrm{Ar})\end{array}$ & $\begin{array}{c}\text { Carbon } \\
\text { dioxide } \\
(\mathrm{mg} / \mathrm{L} \text { as } \\
\left(\mathrm{CO}_{2}\right)\end{array}$ & $\begin{array}{c}\text { Oxygen } \\
(\mathrm{mg} / \mathrm{L} \text { as } \\
\left(\mathrm{O}_{2}\right)\end{array}$ & $\begin{array}{c}\text { Nitrogen } \\
\text { (mg/L as } \\
\left(\mathrm{N}_{2}\right)\end{array}$ & $\begin{array}{c}\text { Nitrous } \\
\text { oxide } \\
(\mathrm{mg} / \mathrm{L} \text { as } \\
\left(\mathrm{N}_{2} \mathrm{O}\right)\end{array}$ & $\begin{array}{c}\text { Hydrogen } \\
\text { sulfide } \\
(\mathrm{mg} / \mathrm{L} \text { as } \\
\left(\mathrm{H}_{2} \mathrm{~S}\right)\end{array}$ & $\begin{array}{c}\text { Methane } \\
\text { (mg/L as } \\
\left(\mathrm{CH}_{4}\right)\end{array}$ \\
\hline $49 \mathrm{~T} 74$ & 231BLBF & $09-05-91$ & 0.7594 & 6.04 & 2.84 & 22.6 & 0.0 & 0.0 & 0.0 \\
\hline $49 \mathrm{U} 73$ & $227 \mathrm{CPCK}$ & $08-22-91$ & .7298 & 12.6 & 0.58 & 19.9 & .0 & .0 & .0 \\
\hline $49 \mathrm{U} 74$ & 227MZCC & $09-04-91$ & .7098 & 3.96 & 3.91 & 20.0 & .0 & .0 & .0 \\
\hline $49 \vee 55$ & $377 \mathrm{CHLH}$ & $02-04-91$ & .7522 & 51.4 & 8.50 & 21.6 & .0 & .0 & .0 \\
\hline $49 \vee 56$ & $377 \mathrm{CHLH}$ & $02-04-91$ & .6654 & 16.3 & 9.13 & 18.1 & .0 & .0 & .0 \\
\hline $49 \mathrm{~V} 58$ & 377CHLH & $02-04-91$ & .8069 & 55.4 & 8.63 & 23.1 & .0 & .0 & .0 \\
\hline $50 \mathrm{U} 133$ & $227 \mathrm{CPCK}$ & 09-03-91 & .7998 & 4.84 & 4.93 & 23.5 & .0 & .0 & .0 \\
\hline \multirow[t]{2}{*}{$51 \mathrm{~T} 29 \mathrm{E}$} & 360 PLLT & $02-05-91$ & .6972 & 44 & 6.68 & 19.1 & .0 & .0 & .0 \\
\hline & & 02-05-91 & .6856 & 46.5 & 6.75 & 18.5 & .0 & .0 & .0 \\
\hline $51 \mathrm{~T} 173$ & $360 \mathrm{PCBC}$ & $02-05-91$ & .7291 & 6.37 & 2.88 & 20.1 & .0 & .0 & .0 \\
\hline $51 \mathrm{U} 10$ & 231PLVL & $02-04-91$ & .7291 & 8.5 & 3.21 & 19.8 & .0 & .0 & .0 \\
\hline $51 \mathrm{U} 97 \mathrm{H}$ & 231PLVL & $02-05-91$ & .7709 & 8.02 & 2.66 & 22.7 & .0 & .0 & .0 \\
\hline 51U103G & 231BLBF & $02-05-91$ & 1.0095 & 12.7 & 5.15 & 35.3 & .0 & .0 & .0 \\
\hline 51U104G & 231PLVL & $02-05-91$ & .7141 & 13.3 & .44 & 21.1 & .0 & .0 & .0 \\
\hline $52 S 18$ & 360LGRV & $08-13-91$ & .7874 & 0.9 & 1.55 & 20.3 & .0 & .0 & .0 \\
\hline $52 S 47$ & 217PTMC & $08-15-91$ & .7334 & 48.8 & 6.26 & 19.3 & .0 & .0 & .0 \\
\hline $52 S 52$ & 217PTMC & $08-21-91$ & .8024 & 7.37 & .02 & 21.9 & .0 & .0 & .0 \\
\hline $52 \mathrm{~T} 66$ & 361QNTC & $09-05-91$ & .7455 & 37.7 & .04 & 20.9 & .0 & .0 & .0 \\
\hline 53T 56 & 377СРМС & $08-14-91$ & .8185 & 11.3 & .07 & 23.0 & .0 & .0 & 2.26 \\
\hline
\end{tabular}

